\title{
EVALUATION OF A STATISTICAL INFILL CANDIDATE SELECTION TECHNIQUE
}

\author{
A Thesis \\ by \\ LINHUA GUAN
}

\begin{abstract}
Submitted to the Office of Graduate Studies of Texas A\&M University

in partial fulfillment of the requirements for the degree of

MASTER OF SCIENCE
\end{abstract}

May 2003

Major Subject: Petroleum Engineering 


\title{
EVALUATION OF A STATISTICAL INFILL CANDIDATE SELECTION TECHNIQUE
}

\author{
A Thesis \\ by \\ LINHUA GUAN \\ Submitted to the Office of Graduate Studies of \\ Texas A\&M University \\ in partial fulfillment of the requirements for the degree of \\ MASTER OF SCIENCE
}

Approved as to style and content by:

Duane A. McVay

(Chair of Committee)

Jerry L. Jensen

(Member)
Richard Gibson Jr.
(Member)

Hans C. Juvkam-Wold

(Interim Head of Department)

May 2003

Major Subject: Petroleum Engineering 


\author{
ABSTRACT \\ Evaluation of a Statistical Infill Candidate Selection Technique. \\ (May 2003) \\ Linhua Guan, M.S., \\ University of Petroleum of China, P.R. China \\ Chair of Advisory Committee: Dr. Duane A. McVay
}

Quantifying the drilling or recompletion potential in producing gas basins is often a challenging problem, because of large variability in rock quality, well spacing, and well completion practices and the large number of wells involved. Complete integrated reservoir studies to determine infill potential are often too time-consuming and costly for many producing gas basins.

In this work we evaluate the accuracy of a statistical moving-window technique that has been used in tight-gas formations to assess infill and recompletion potential. The primary advantages of the technique are its speed and its reliance upon well location and production data only.

We used the statistical method to analyze simulated low-permeability, 100-well production data sets, then compared the moving-window infill-well predictions to those from reservoir simulation. Results indicate that moving-window infill predictions for individual wells can be off by more than 50\%; however, the technique accurately predicts the combined infill-production estimate from a group of infill candidates, often to within $10 \%$.

We found that the accuracy of predicted infill performance decreases as heterogeneity increases and increases as the number of wells in the project increases. The cases evaluated in this study included real-world well spacing and production rates and a significant amount of depletion at the infill locations. Because of its speed, accuracy and reliance upon readily available data, the moving window technique can be a useful screening tool for large infill development projects. 


\section{DEDICATION}

This thesis is dedicated to:

My wife, Haiying Zhang, who gives me love and support all the time as well as takes care of our daughter and our family living.

My family members in China, who always give me love, encouragement and support;

My committee chairman, Dr. D.A. McVay, who gave me the precious opportunity to pursue my master's degree at Texas A\&M University. His financial support is the most important source that helps me to finish my study here. He has opened my mind in doing this research.

My committee member, Dr. L. J. Jensen, who has given me lots of support of my study in the USA. 


\section{ACKNOWLEDGEMENTS}

I would like to thank the following for their contributions toward the success of this thesis:

Dr. Duane A. McVay, for his guidance, encouragement, tireless effort, patience and financial support to help me to complete this thesis;

Dr. Jerry L. Jensen, for his guidance, help in my study, help getting financial support, and for his kindly serving as a member of my advisory committee;

Dr. Richard Gibson Jr. for his interest and kindly serving as a member of my advisory committee;

Dr. Thomas A. Blasingame, for his kind help and concern about my study and living; and

My many friends, who gave me help, concern and encouragement all the time. 
TABLE OF CONTENTS

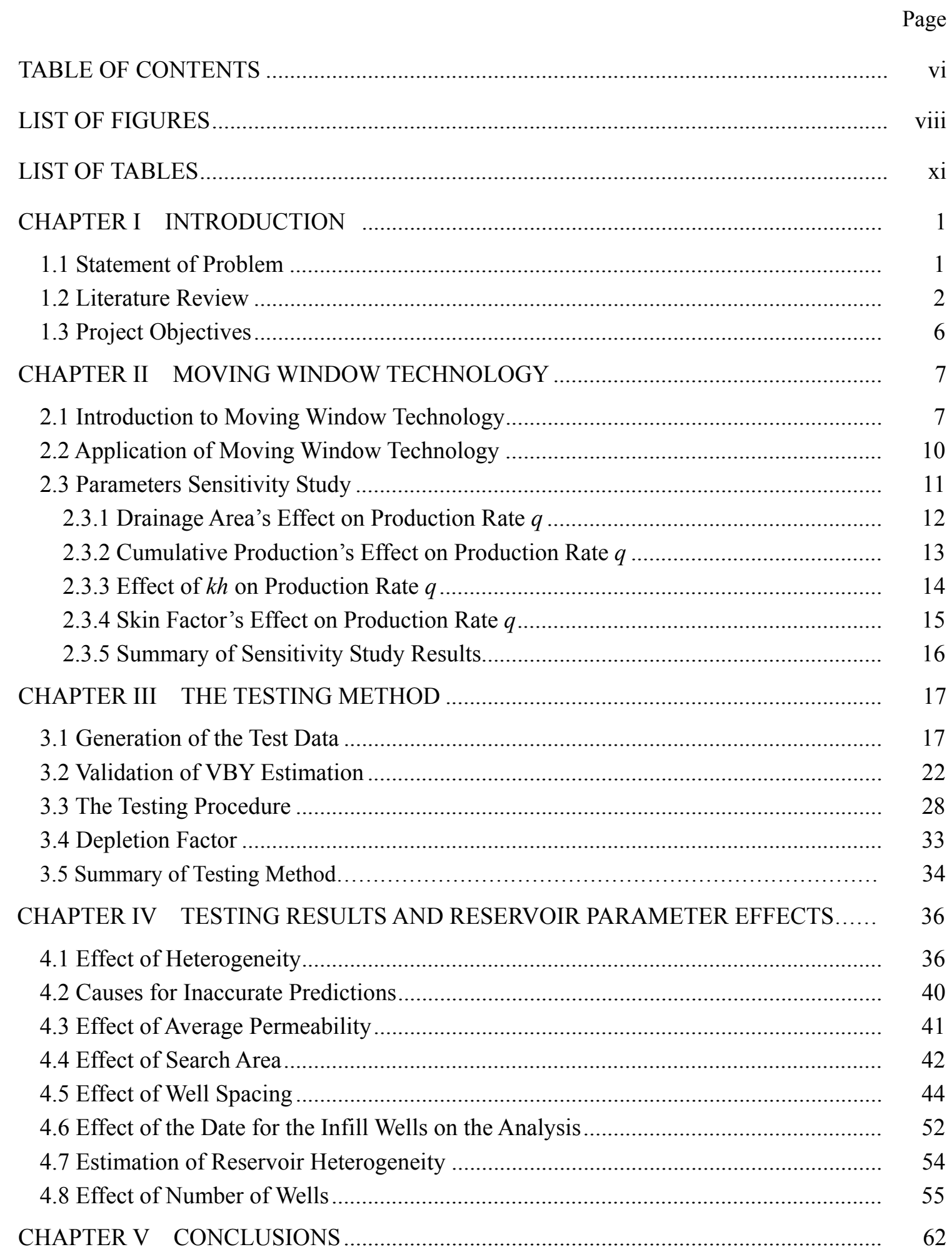


CHAPTER VI DISCUSSION OF LIMITATIONS AND FUTURE WORK ….................. 63

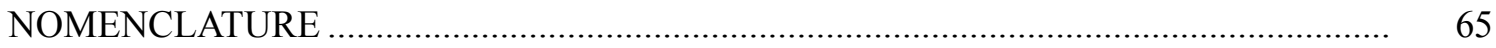

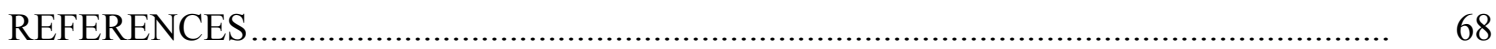

APPENDIX A $\quad$ MOSAIC 4D MODEL FOR GAS RESERVOIR .......................................

VITA 


\section{LIST OF FIGURES}

Page

Fig. 1.1 Technology improved first year cumulative production ${ }^{7}$

Fig. 2.1 Diagram of the moving window technology showing how the window moves across area

Fig. 2.2 Illustration of $\mathrm{BY}^{5}$

Fig. 2.3 Best Year vs. 10-year cumulative production ${ }^{5}$

Fig. 2.4 Drainage area does not have much effect on production $q$

Fig. 2.5 Cumulative production does not have much effect on production $q$

Fig. 2.6 Production $q$ is strongly dependent on $k h$.

Fig. 2.7 Sensitivity study shows that $k h$ has greatest effect on $q$ calcualation..... 16

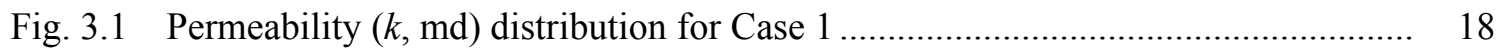

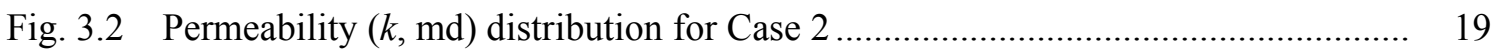

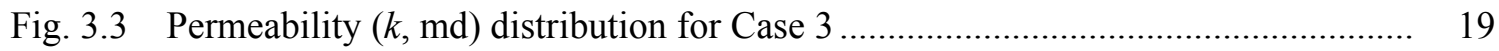

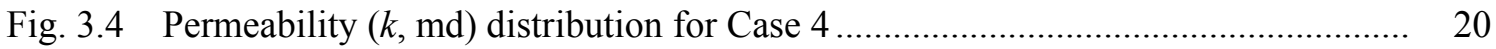

Fig. 3.5 Histogram of current well spacing for the 100 -well cases ................................... 21

Fig. 3.6 Histogram of date of first production for the 100-well cases ................................ 21

Fig. 3.7 Comparison of VBY from simulation and Mosaic technology for Case 1. VBY from Mosaic technology shows little deviation from simulation for Case 1....... 23

Fig. 3.8 Comparison of VBY from simulation and Mosaic technology for Case 2. VBY from Mosaic technology correlates well with simulation for Case 2.........

Fig. 3.9 Increased heterogeneity in Case 3 introduces scatter between VBY from Mosaic technology and simulation

Fig. 3.10 High heterogeneity of Case 4 results in great scatter in VBY between Mosaic technology and simulation

Fig. 3.11 VBY serves well as a proxy for $k h$ for Case 1 


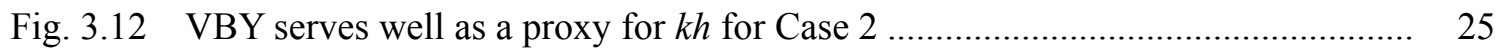

Fig. 3.13 Increased heterogeneity reduces value of VBY as a proxy for $k h$ for Case $3 \ldots \ldots . .26$

Fig. 3.14 High heterogeneity of Case 4 severely reduces effectiveness of VBY as a proxy for $k h$.

Fig. 3.15 New-well 1-year cumulative production corresponds well with incremental field 1-year production

Fig. 3.16 Simulation well regions

Fig. 3.17 Max_New_Well_BY vs. Avg_New_Well_BY for Case 3

Fig. 3.18 Infill BY vs. Max_New_Well_BY for Case 3 ................................................... 32

Fig. 3.19 Infill BY vs. Avg_New_Well_BY for Case 3 …............................................... 33

Fig. 4.1 Comparison of infill BY from Mosaic and simulation for Case 1. Infill BY from Mosaic technology shows little deviation from simulation for this case

Fig. 4.2 Comparison of infill BY from Mosaic and simulation for Case 2. Infill BY from Mosaic technology correlates well with simulation for this case.

Fig. 4.3 Increased heterogeneity in Case 3 introduces scatter between infill BY from Mosaic and simulation.

Fig. 4.4 High heterogeneity of Case 4 results in great scatter in infill BY from Mosaic and simulation

Fig. 4.5 Infill BY from Mosaic shows low correlation with simulation for $1.0 \mathrm{md}$ homogeneous reservoir

Fig. 4.6 Relative error of VBY and infill BY vs. search area for Case 3........................ 43

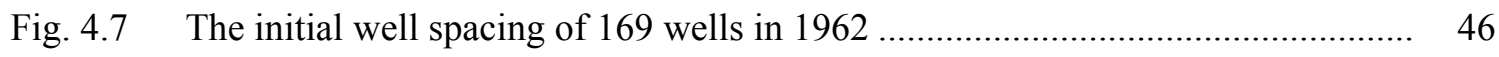

Fig. 4.8 The well spacing after the first round of infill-drilling in 1972 .......................... 47

Fig. 4.9 The well spacing after the second round of infill-drilling in $1982 \ldots \ldots \ldots \ldots \ldots \ldots \ldots \ldots . . . . . . . . . . . .48$

Fig. 4.10 Absolute relative error with the domain size .................................................. 52 
Fig. 4.11 Effect of the timing of the infill wells on the moving window technology $($ data from Case 1a) ........................................................ 54

Fig. 4.12 Variability in production data related to variability in permeability ..................... 55

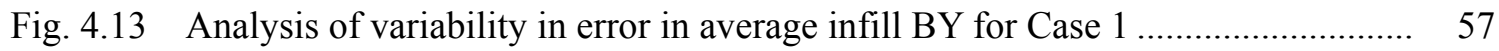

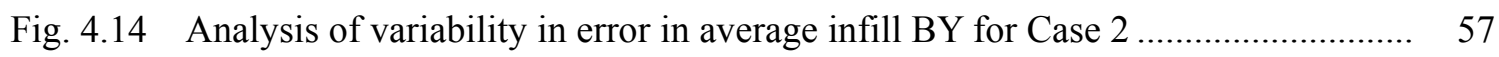

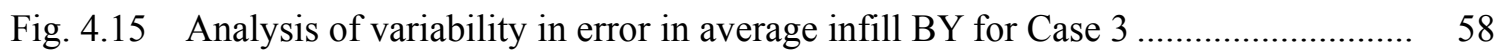

Fig. 4.16 Analysis of variability in error in average infill BY for Case $4 \ldots \ldots \ldots \ldots \ldots \ldots \ldots \ldots \ldots . . . . . . . . . . . . .58$

Fig. 4.17 Variability in percent error in average infill BY for four 100-well cases increases with reservoir heterogeneity and decreases with number of wells........ 59

Fig. 4.18 Variability in absolute error in average infill BY and theoretical model fit .......... 60 


\section{LIST OF TABLES}

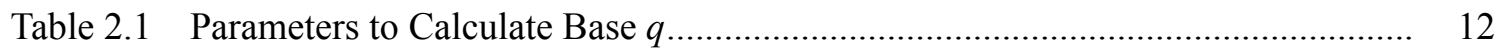

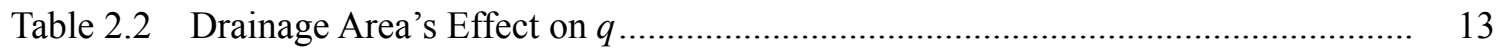

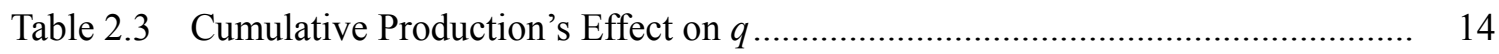

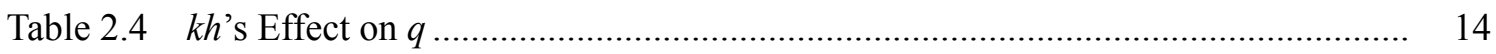

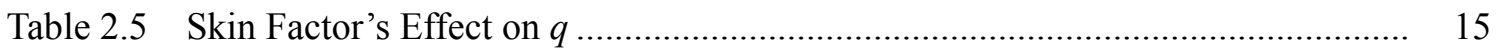

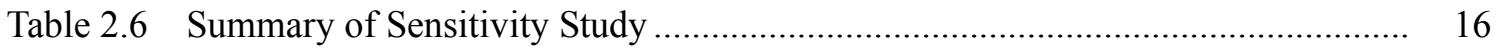

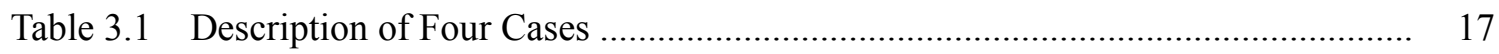

Table 3.2 VBY Comparisons From Mosaic and Simulation Methods ................................ 27

Table 3.3 Depletion Factors of Four Cases...................................................................... 34

Table 4.1 Comparison From Mosaic and Simulation Methods …................................... 40

Table 4.2 Effect of Search Area on Relative Error for Case 3 ............................. 43

Table 4.3 Results From Uniform Spacing With Boundary Wells........................... 49

Table 4.4 Results From Uniform Well Spacing Without Boundary Wells.................. 50

Table 4.5 Effect of Date for Infill Wells on Moving Window Technology................. 53

Table 4.6 Number of Wells Required for Desired Level of Varibility .................... 61 


\section{CHAPTER I}

\section{INTRODUCTION}

\subsection{Statement of Problem}

The best way to determine infill-drilling potential in a gas basin is to conduct a complete reservoir evaluation involving geological, geophysical, and reservoir analyses and interpretations. This includes developing a geological model of the study area, estimating distributions of static reservoir properties such as porosity and permeability, constructing and calibrating a reservoir simulation model of the area, and then using the reservoir model to predict future production and reserves at potential infill-well locations.

While it may be accurate, this approach can be prohibitively time-consuming and expensive. The variation of well spacing, the local and regional variation in rock quality, and the benefits of improved completion and production technologies often cloud the extent of reservoir depletion. All these will make the quantification of infill potential very difficult.

So for some large, low-permeability gas basins with large data sets (sometimes over 1,000 wells) and complex geology, the cost and time requirements of a conventional reservoir evaluation study are not acceptable.

At the same time, with the increasing demand for natural gas, more and more gas basins within North America are undergoing infill drilling. It is not uncommon for a company to have hundreds or thousands of infill candidates to choose from. So when we

This thesis follows the style of the SPE Reservoir Evaluation and Engineering. 
are faced with little time and large data sets, we need a fast method to evaluate infill prospects.

\subsection{Literature Review}

French et al. ${ }^{1}$ used empirical infill-drilling forecast models and infill-well economics for Permian Basin Clearfork and San Andres waterflood units. The purpose was to study infill-drilling performance and derive empirical infill recovery forecast models for the Clearfork and San Andres formations. The forecasts matched actual field data reasonably well according to $\mathrm{R}^{2}$ (from 0.7792 to 0.995 ) and $\mathrm{F}$ values (from 29 to 1506) of the forecast models.

Later, $\mathrm{Wu}$ et $a .^{2,3}$ applied fuzzy logic, nonlinear regression, nonparametric regression, and neural-network models to forecast primary ultimate oil recovery and infill-drilling ultimate oil recovery. Their research helped explain the relative importance of dominant reservoir characteristics and operational variables and how to forecast the recoveries for infill drilling with similar geologic settings.

McCain et al. ${ }^{4}$ first used a statistical method to determine infill potential in a complex, low-permeability gas reservoir. Their method provided an unbiased means of comparing well performance, selecting areas for advanced analysis, and defining the areal locations where specific conclusions apply. The paper presented a practical means of applying advanced analysis to the entire field. However, the authors also included tedious $\log$ interpretations in their research to assure the accuracy of the net pay thickness calculation.

Voneiff and Cipolla ${ }^{5}$ enhanced this statistical method in their research on the Ozona field and termed it "moving domain" technology. This technology evaluates when the wells are produced, where the wells are located, and how much they produce to find evidence of depletion and determine the effective well spacing. The core of this 
approach is a moving window of local studies that draw statistical conclusions about well performance, depletion, and undrained acreage.

The moving domain technology includes a set of empirically derived approximations, comparisons, and statistical tests that attempt to mimic what a reservoir engineer does when faced with a single infill-location evaluation. It looks at surrounding well performance, compares the new wells to the old wells for signs of depletion, calculates effective well density and then estimates undrained acreage and infill reserves. The input data required for the method are only the wells' locations and production profiles.

The moving domain technology described by Voneiff and Cipolla can be applied in two phases. The first phase is a scoping study that can be completed in a matter of weeks even for over 1,000 wells. The results of this phase provide the preliminary infill estimates, areas of depletion, and areas required for detailed conventional engineering. The second phase involves detailed engineering evaluation to calculate drainage areas, undrained areage, recovery per acre, and infill reserves.

The moving "domain" is a moving study area that contains approximately 5 to 15 wells. The moving window analysis method is based on three assumptions:

- The reservoir properties do not change significantly within any moving window throughout the study area.

- Completion and production technologies used in each well are the same, no matter when the well was drilled and completed.

- At least a few wells in each part of the field have sufficient production history to experience boundary-dominated flow. This is required to calculate drainage area and recovery per acre. 
When we look at the moving domain technology as a whole, we find three limitations with this technology under the three assumptions above.

The first assumption is reasonable for many reservoirs. But there are reservoirs whose properties change dramatically within small areas. Some labyrinth-type reservoirs are composed of narrow channel-fill bodies and major changes in rock properties can occur between sand units in jigsaw-puzzle reservoirs. ${ }^{6}$ We cannot put much confidence in the estimation results from the moving domain technology in those cases.

The second limitation is that if we find the performance of new wells was worse than old wells, we do not know whether depletion or variation in rock properties caused the drop in production in the wells. Moreover, the changes in completion and production technology over a long time frame could also mask the effect of depletion.

The first-year cumulative production data $^{7}$ of the wells in Hugoton gas field show that the completion technologies used in 1994 apparently outperform those used in 1991. Fig. 1.1 shows the results in detail. The average reservoir pressure in 1994 had decreased approximately 20\% from the value measured in 1991. Apparently, technology changes masked the effects of depletion in this field.

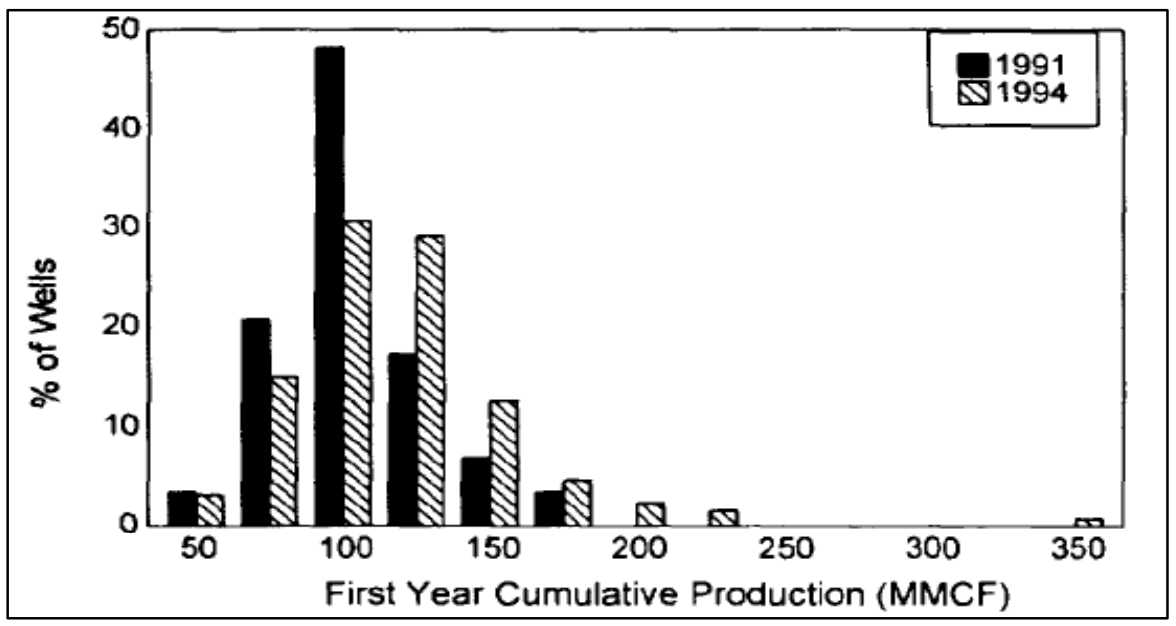

Fig. 1.1 Technology improved first year cumulative production ${ }^{7}$ 
The third limitation is that this technology requires at least a few wells in each part of the field and that have sufficient production history to experience boundary-dominated flow. Only when this is true can we accurately calculate the drainage area and recovery per acre. However, some low-permeability reservoirs take from 9 to 14 years to reach boundary-dominated flow. ${ }^{5}$ Thus, we may not be able to apply moving window technology in some gas fields with short production histories, e.g., less than 10 years.

Despite these assumptions and limitations, this moving domain technique has been applied to the Ozona (Canyon) gas sands, ${ }^{5}$ Milk River formation in Canada, ${ }^{8}$ Cotton Valley in east Texas, ${ }^{4,9}$ Mesaverde formation in the San Juan Basin, ${ }^{9}$ and the Morrow formation in the Permian Basin ${ }^{9}$ to quantify infill potential. But all these studies did not give any quantitative assessment of their estimation results.

Hudson et al. ${ }^{9}$ note when they apply the moving domain technique to Mesaverde, Morrow, and Cotton Valley formations, "Based on our experience, we have found that comparisons between actual and predicted individual infill well performance can vary significantly. Therefore, ranking infill candidates on predicted individual well performance may not necessarily yield the best overall results.... Alternatively, when considering an infill drilling program as a whole, we have found that the predicted performance for groups of wells can be quite accurate." They did not give any further quantitative information on the accuracy of their results. In particular, Hudson et al. ${ }^{9}$ did not quantify their expressions "very significantly" and "quite accurate."

Kyte and Meehan ${ }^{10}$ applied the moving window method to the Austin chalk. They estimated ultimate recoveries (EUR) on a barrels/acre basis by calculating every well's EUR and drill density. Then they analyzed the depletion and infill potential. Finally, they used a neural network to study the effects of parameters such as first production date and structure of the Austin chalk in some of the study areas. Their 
results indicated that the optimum interwell distances for horizontal wells could be determined by comparing effective well densities in the areas not yet being fully drained. This research extended the application of the moving window method from vertical to lateral wells.

\subsection{Project Objectives}

As an alternative approach to conducting complete reservoir evaluations for large, tight-gas basins with large data sets, various authors have used empirical or statistical analyses to model variable well performance. ${ }^{1-5,8-10}$ In particular, the moving domain technology, described by McCain et al. ${ }^{4}$ and enhanced by Voneiff and Cipolla ${ }^{5}$ has been applied to several tight gas reservoirs to quantify infill potentials. ${ }^{4,5,8,9}$

Although the moving domain technology has been applied to several tight gas reservoirs, we could not find any quantitative assessment of the validity and accuracy of the technique in the literature.

The objective of this work is to quantify the accuracy of moving window technology in predicting infill well potential. We did this by calculating the infill-well performance with the moving window method from simulated data and comparing the results with those from the reservoir simulator. The moving window technology we used in our work is an extension of the method described by Voneiff and Cipolla. ${ }^{5}$ 


\section{CHAPTER II}

\section{MOVING WINDOW TECHNOLOGY}

In this chapter we introduce some basic concepts and review the reservoir model used in the moving window technology. In Appendix A we show the derivation of the detailed reservoir models employed in the technology.

\subsection{Introduction to Moving Window Technology}

The technique employed in this work, herein referred to as moving window or Mosaic technology, is an extension of the method described by Voneiff and Cipolla. ${ }^{5}$ This technology consists of a multitude of local analyses, each in an areal window centered around an existing well (Fig. 2.1).

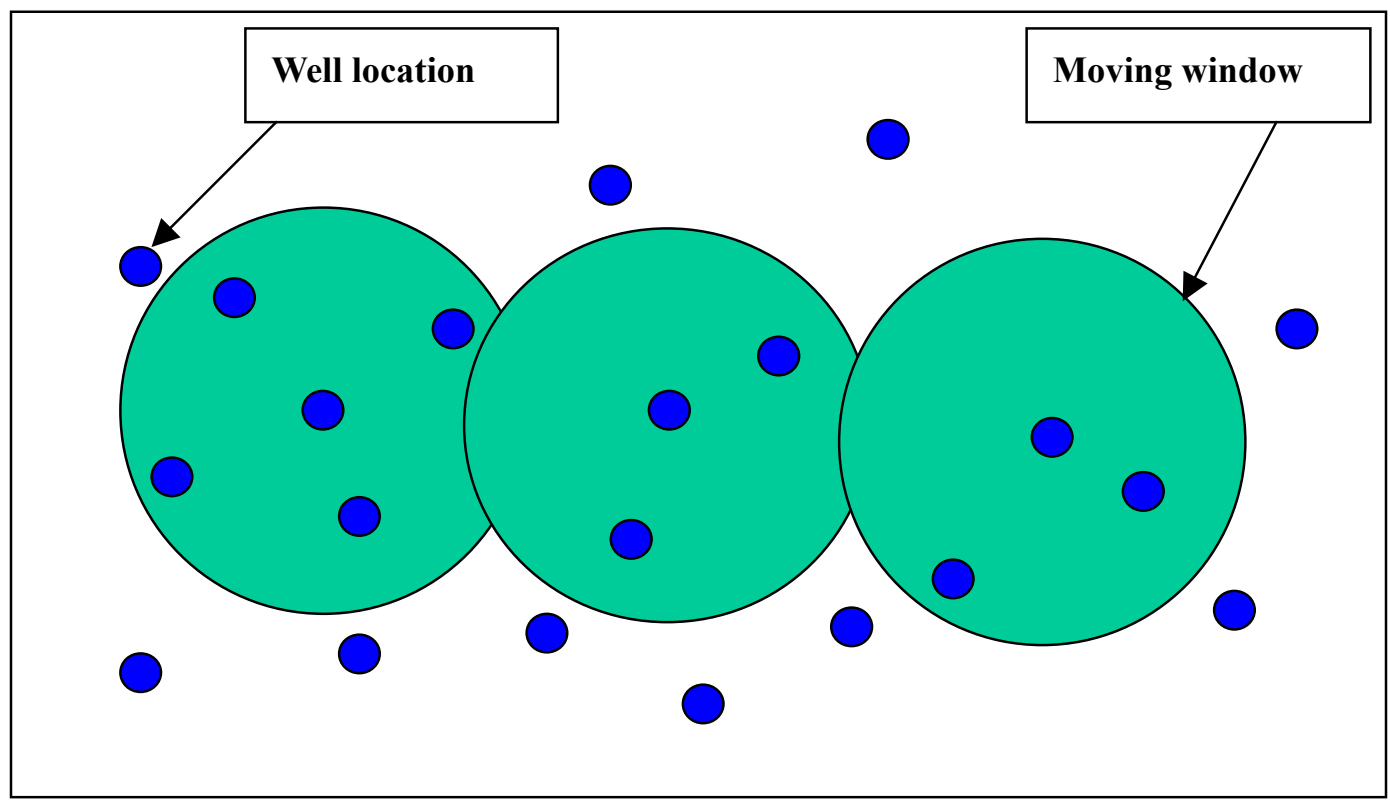

Fig. 2.1 Diagram of the moving window technology showing how the window moves across area. 
The moving domain technique used by McCain et al ${ }^{4,5,8,9}$ is not a very rigorous model-based analysis. The technique includes a 2D regression of best year of production vs. time to find areas of depletion.

In this work, however, a more rigorous, model-based analysis is employed in each moving window. The model is based on a combination of the material-balance equation and the pseudosteady-state flow equation, simplified by assuming that many properties are constant within an individual window. Eq. 2-1 is the detailed reservoir model that we used in the moving window technology (see Appendix A for the derivation of this equation.).

$$
\ln q=\ln (k h)+C_{1}+\ln \left(p_{i}-p_{w f}-C_{2} \frac{G_{p}}{A}\right)-\ln \left(\ln (\sqrt{A})+C_{3}\right),
$$

Simplifying the equation above, we get Eq. 2-2. This equation is a linear 4D regression equation that is applied within each window:

$$
\mathrm{BY}=\mathrm{f}\left(\mathrm{VBY}, G_{p} / A, A\right),
$$

where

$\mathrm{BY}=$ best year, the highest 12 consecutive months of production divided by 12 (Fig. 2.2). BY serves as an indicator for estimated ultimate recovery (Fig. 2.3).

$\mathrm{VBY}=$ virgin best year, the BY of a well at virgin conditions. Depletion effects are removed by computing the BY of a local area at a time before depletion using a 2D regression of BY vs. well start date. VBY is used as a proxy for $k h$ in the pseudosteady-state flow equation.

$G_{p} / A=$ cumulative production divided by well spacing.

$A=$ area of voronoi polygon around each well based on $\mathrm{x} / \mathrm{y}$ well locations. This is used as a proxy for drainage area in the pseudosteady state flow equation and material balance equation. 


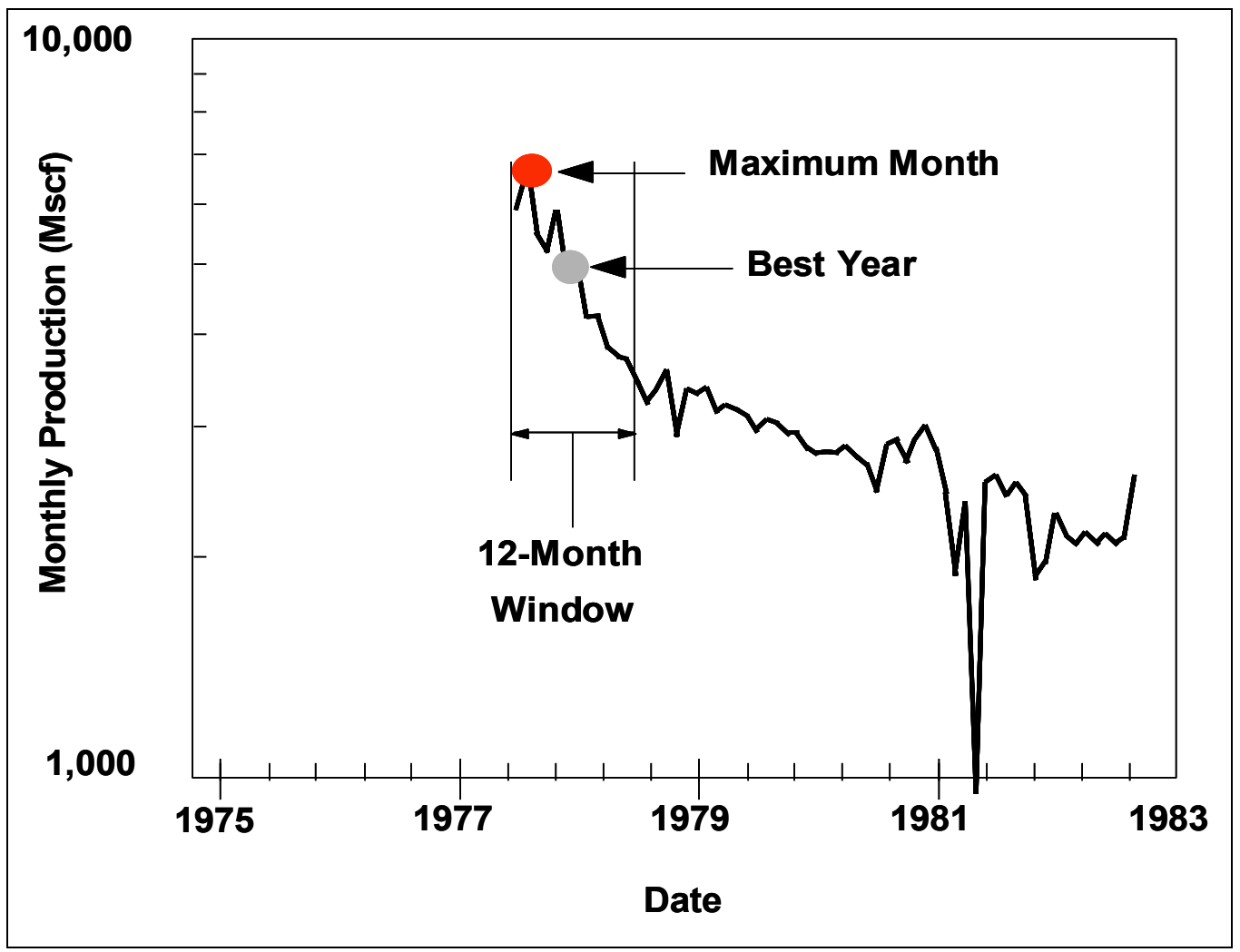

Fig. 2.2 Illustration of $\mathrm{BY}^{5}$.

Voneiff and Cipolla $^{5}$ validated the use of BY (Fig. 2.2) as an indicator for estimated ultimate recovery (EUR) in their research on Ozona gas sands. Fig. 2.3 shows good correlation between the best-year indicator and long-term performance. This figure includes 962 wells with 10 years' cumulative production data in the Ozona gas sand. From this figure we know that we can use BY as an indicator of EUR.

Detailed derivations of the moving window models for gas reservoirs are presented in Appendix A. 


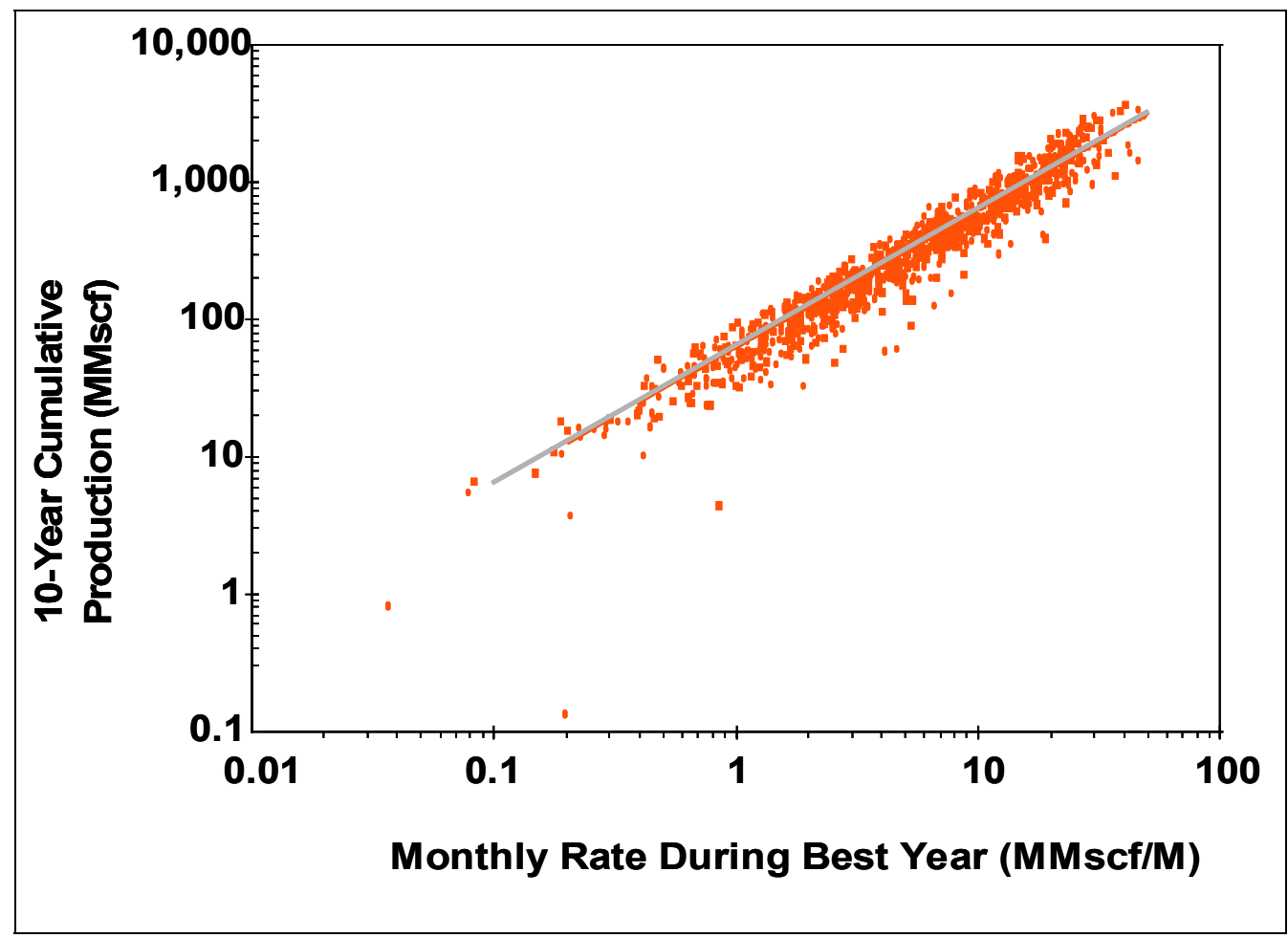

Fig. 2.3 Best Year vs. 10-year cumulative production ${ }^{5}$.

\subsection{Application of Moving Window Technology}

This technology evaluates the study field as a mosaic of overlapping local studies, not as a single study. Each well in the study area is evaluated using the 4D reservoir model to compare its performance with the production of neighbor wells. The result of this analysis is a prediction of BY for a new infill well offsetting each existing well.

The first step of the moving window technology is performing a $2 \mathrm{D}$ regression. The $2 \mathrm{D}$ regression is of $\mathrm{BY}$ vs. time, and the objective is to determine the VBY used in the subsequent 4D regression.

The next step is the $4 \mathrm{D}$ regression. The $4 \mathrm{D}$ regression correlates $\mathrm{BY}$ vs. VBY, cumulative withdrawal per acre, and well spacing. The regression coefficients for each window are determined by regressing these parameters for the wells within each window. Once the regression equation coefficients are determined for each window, performance 
can be estimated for infill wells by substituting the appropriate values for candidate infill well conditions.

The moving windows are limited in size, e.g., 3,000 acres, and generally contain 5 to 20 wells. If the number of wells in a window is less than a minimum value, e.g., 3 to 5 , then a regional or global regression is used instead of a local regression.

The infill well expectations are computed on the basis of existing well data and processed using the "Mosaic_4D.xls" spreadsheet. This spreadsheet reads the whole field production dataset and processes the data in small subsets. The final study results are stored in the table called "Infill_Bestyear" in the database.

\subsection{Parameters Sensitivity Study}

The moving window reservoir model that was derived by Voneiff for gas reservoirs is Eq. 2-1.

$$
\ln q=\ln (k h)+C_{1}+\ln \left(p_{i}-p_{w f}-C_{2} \frac{G_{p}}{A}\right)-\ln \left(\ln (\sqrt{A})+C_{3}\right),
$$

where

$$
\begin{aligned}
& C_{1}=\quad-\ln (141.2 B \mu) \\
& C_{2}=0.00742 \frac{\pi B}{c_{t} h \phi} \\
& C_{3}=\quad 0.40428+s+0.5 \times \ln \left(C_{A} r_{w}{ }^{2}\right)
\end{aligned}
$$

Here we want to determine the effect of reservoir permeability, net pay thickness, cumulative production, skin factor, and well spacing on the calculation of best year. We did this by conducting a simple sensitivity study of these parameters. 
The parameters that we used to calculate the production rate, $q$, are in Table 2.1 . We define this case as the base case and the production $q$ here is the base production, $q_{\text {base }}$. The $q_{\text {base }}$ we calculated is 246.7 Mscf/D.

Table 2.1-Parameters to Calculate Base $q$

\begin{tabular}{|c|c|c|c|}
\hline Parameter & Value & Parameter & Value \\
\hline$p_{i}, \mathrm{psi}$ & 1,100 & $T,{ }^{\circ} \mathrm{R}$ & 550 \\
\hline$p_{\text {wf }} \mathrm{psi}$ & 250 & $h, \mathrm{ft}$ & 40 \\
\hline$G_{p}, \mathrm{Mscf}$ & 10,000 & $s$, & 0 \\
\hline$A$, acres & 640 & $B, \mathrm{RB} / \mathrm{STB}$ & 0.005 \\
\hline$k, \mathrm{md}$ & 0.2 & $C_{t}, \mathrm{psia}^{-1}$ & 0.0001 \\
\hline$\mu, \mathrm{cp}$ & 0.017 & $\phi, \mathrm{fraction}$ & 0.2 \\
\hline$Z$ & 1 & $r_{\mathrm{w}}, \mathrm{ft}$ & 0.3 \\
\hline
\end{tabular}

\subsubsection{Drainage Area's Effect on Production Rate $q$}

To find the drainage area's effect, we kept all other parameters the same as they are in Table 2.1. We increased the drainage area from 160 acres $(160 / 640=0.25)$ to 6,400 acres $(6,400 / 640=10)$ and calculated the $q$, for each. We calculated $q / q_{\text {base, }}$, the ratio of the new $q$ and $q_{\text {base. }}$. We found that effect of drainage area on $q$ is not significant. The drainage area increased 40 -fold, but the $q$ decreased less than one-fold.

Table 2.2 and Fig. 2.4 show the result of drainage area's effect on the production q. From this table we know that when we increase the well spacing to 10 times of the original well spacing, the production will only decrease about $28 \%$. 
Table 2.2-Drainage Area's Effect on $q$

\begin{tabular}{|c|c|c|c|c|c|c|}
\hline$A$ & $1 / 4 A$ & $1 / 2 A$ & $1 A$ & $2 A$ & $4 A$ & $10 A$ \\
\hline$q / q_{\text {base }}$ & 1.36 & 1.14 & 1.00 & 0.89 & 0.81 & 0.72 \\
\hline
\end{tabular}

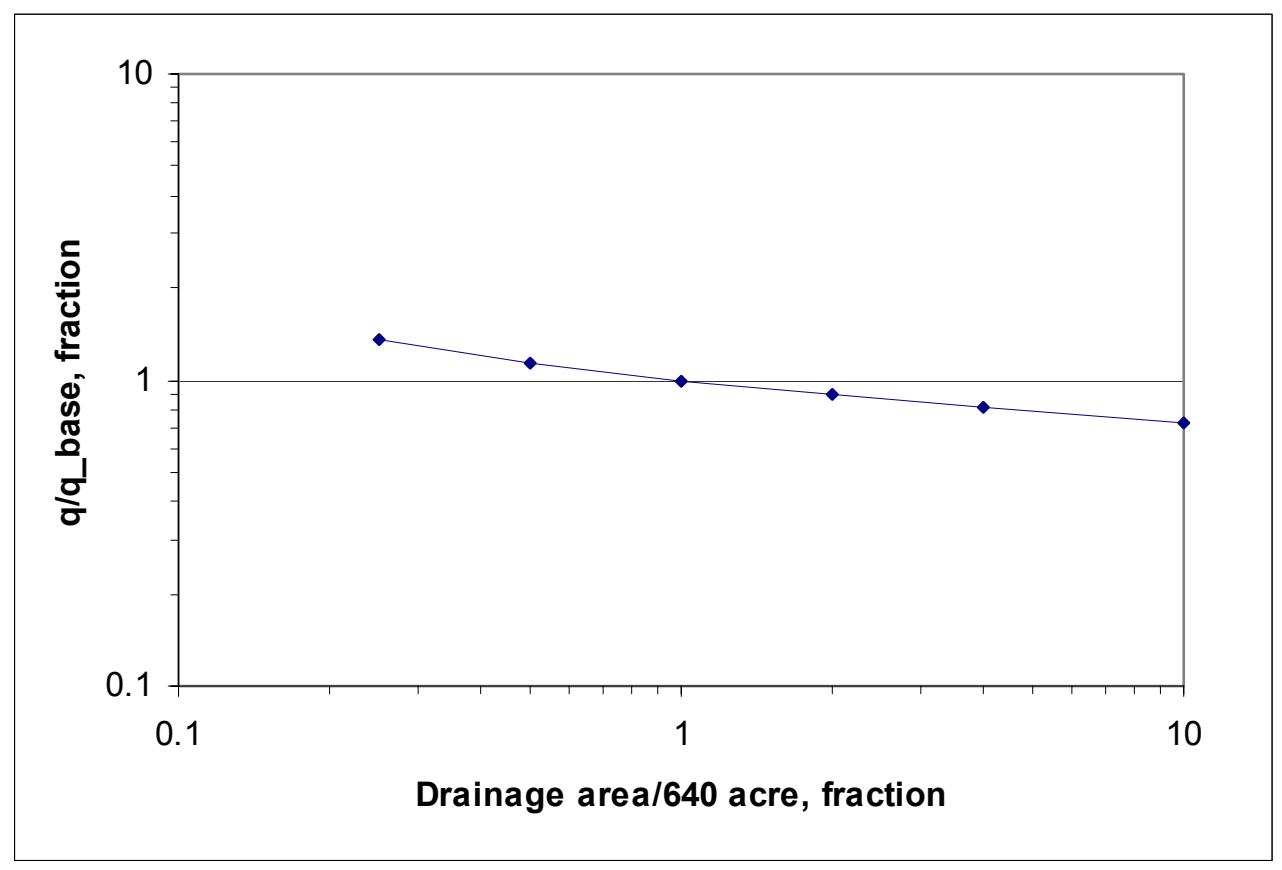

Fig. 2.4 Drainage area does not have much effect on production $q$.

\subsubsection{Cumulative Production's Effect on Production Rate $q$}

Table 2.3 and Fig. 2.5 show the result of cumulative production's effect on the normalized flow rate, $q / q_{\text {base }}$. We found that the changing of cumulative production does not have much effect on the $q$ calculation. We increased the cumulative production 40-fold, but $q / q_{\text {base }}$ just changed $35 \%$.

When we check Eq. 2-1, we find that $C_{2} / A$ is a very small number, just 0.002 in this sensitivity study, which decreases the effect of cumulative production. If the cumulative production is a very large number, it will have a larger effect on the production $q$. 
Table 2.3-Cumulative Production's Effect on $q$

\begin{tabular}{|c|c|c|c|c|c|c|}
\hline$G_{p}$ & $G_{p} / 4$ & $G_{p} / 2$ & $1 G_{p}$ & $2 G_{p}$ & $4 G_{p}$ & $10 G_{p}$ \\
\hline$q / q_{\text {base }}$ & 0.98 & 0.99 & 1 & 1.02 & 1.09 & 1.33 \\
\hline
\end{tabular}

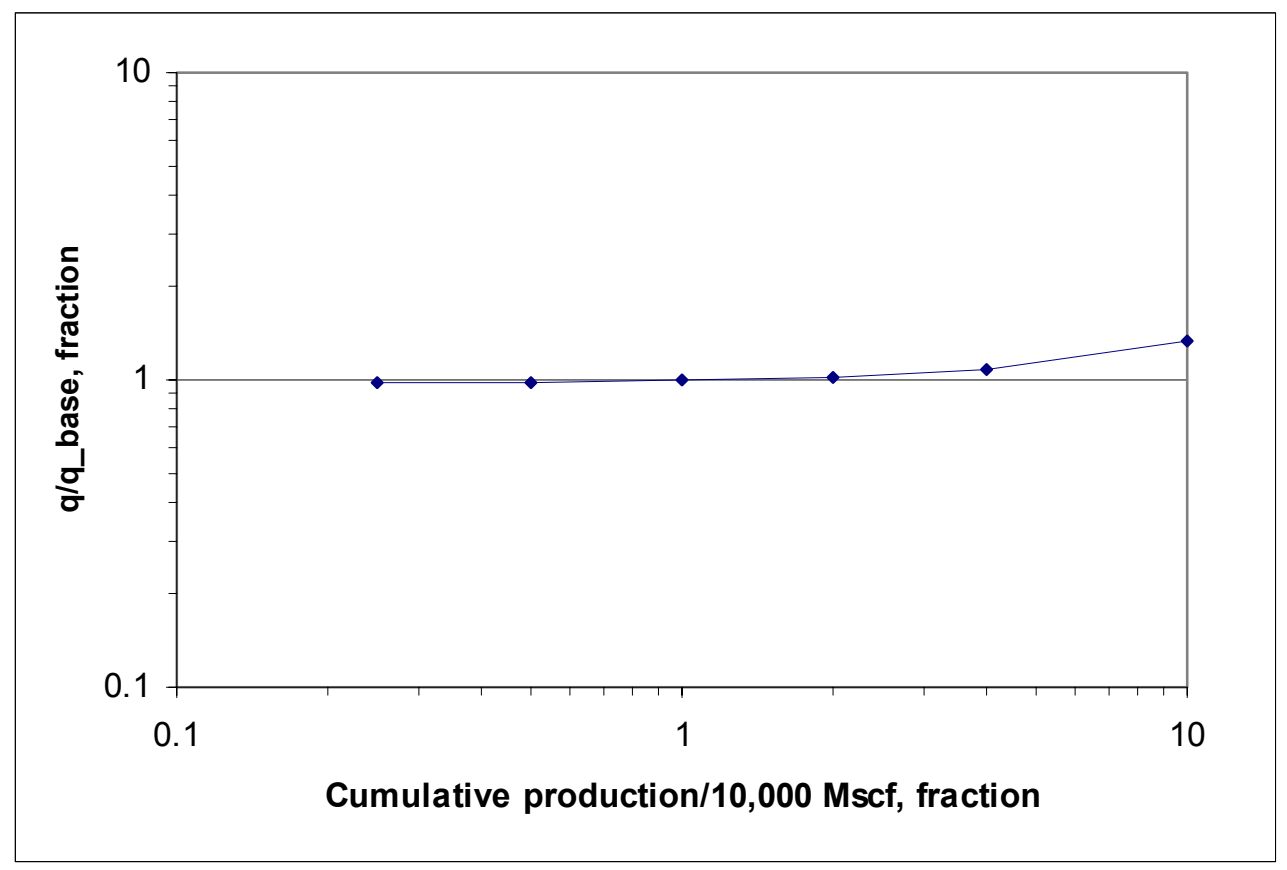

Fig. 2.5 Cumulative production does not have much effect on production $q$.

\subsubsection{Effect of $k h$ on Production Rate $q$}

By inspection the detailed governing equation Eq. A-24 (P71), we can easily find the 1:1 relationship between $k$ and $q$, but the relationship between $h$ and $q$ is also close to 1:1. Table 2.4 and Fig. 2.6 show the result of $k h$ 's effect on the normalized flow rate, $q / q_{\text {base }}$, by changing of $h$. So the production $q$ is highly dependent on the value of $k h$.

Table 2.4-kh's Effect on $q$

\begin{tabular}{|c|c|c|c|c|c|c|}
\hline$k h$ & $k h / 4$ & $k h / 2$ & $k h$ & $2 k h$ & $4 k h$ & $10 k h$ \\
\hline $\begin{array}{c}q / q_{\text {base }}(k \text { remains } \\
\text { constant, } h \text { changes })\end{array}$ & 0.27 & 0.51 & 1.00 & 1.97 & 3.91 & 9.76 \\
\hline
\end{tabular}




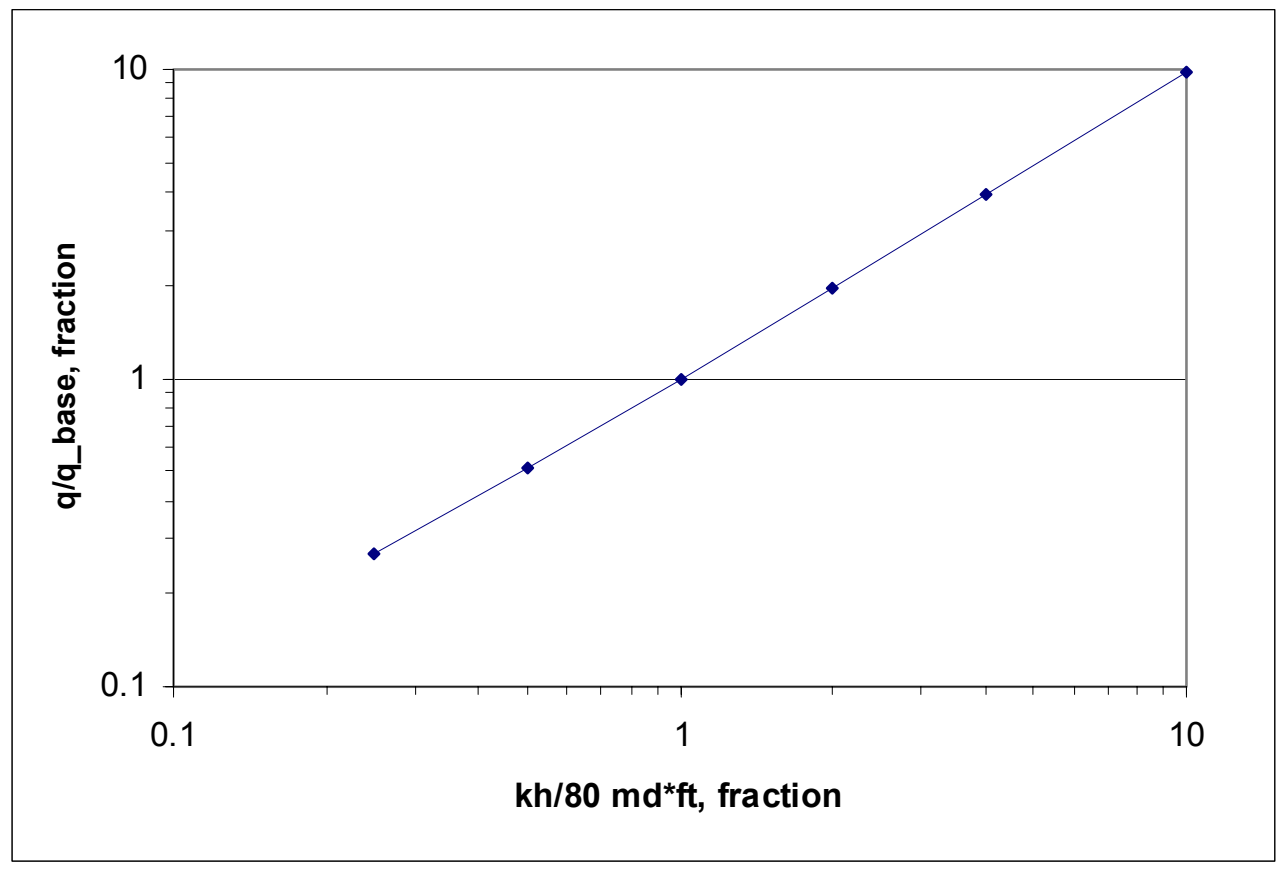

Fig. 2.6 Production $q$ is strongly dependent on $k h$.

\subsubsection{Skin Factor's Effect on Production Rate $q$}

Table 2.5 shows the result of skin factor's effect on $q$. It seems that the skin factor has little effect on the $q$ calculation in the moving window technology. So we can be sure that the completion technology changes will not have too much impact on the results of the moving window technology.

Table 2.5-Skin Factor's Effect on $q$

\begin{tabular}{|c|c|c|c|c|c|c|}
\hline$s$ & 4 & 2 & 0 & -1 & -2 & -4 \\
\hline$q / q_{\text {base }}$ & 0.96 & 0.98 & 1.00 & 1.01 & 1.02 & 1.05 \\
\hline
\end{tabular}




\subsubsection{Summary of Sensitivity Study Results}

Table 2.6 and Fig. 2.7 show the effects of drainage area, cumulative production, $k h$, and skin factor on the production calculation. These results indicate that the accuracy of moving window technology will mainly depend on the value of $k h$. Neither drainage area, cumulative production, nor skin factor have much effect on the production $q$ calculation. In the moving window technology, we do not estimate $k h$ directly. We use another parameter, VBY, as a proxy for the $\mathrm{kh}$.

The sensitivity results support the use of well spacing as a proxy for drainage area because of its small effect on the production calculation.

Table 2.6-Sumary of Sensitivity Study

\begin{tabular}{|c|c|c|c|c|c|}
\hline Parameter & Parameter/4 & Parameter/2 & Parameter/1 & Parameter 2 & Parameter*4 \\
\hline$A$ & 1.36 & 1.14 & 1.00 & 0.89 & 0.81 \\
\hline$G_{p}$ & 0.98 & 0.99 & 1.00 & 1.02 & 1.09 \\
\hline$k h$ & 0.27 & 0.51 & 1.00 & 1.97 & 3.91 \\
\hline
\end{tabular}

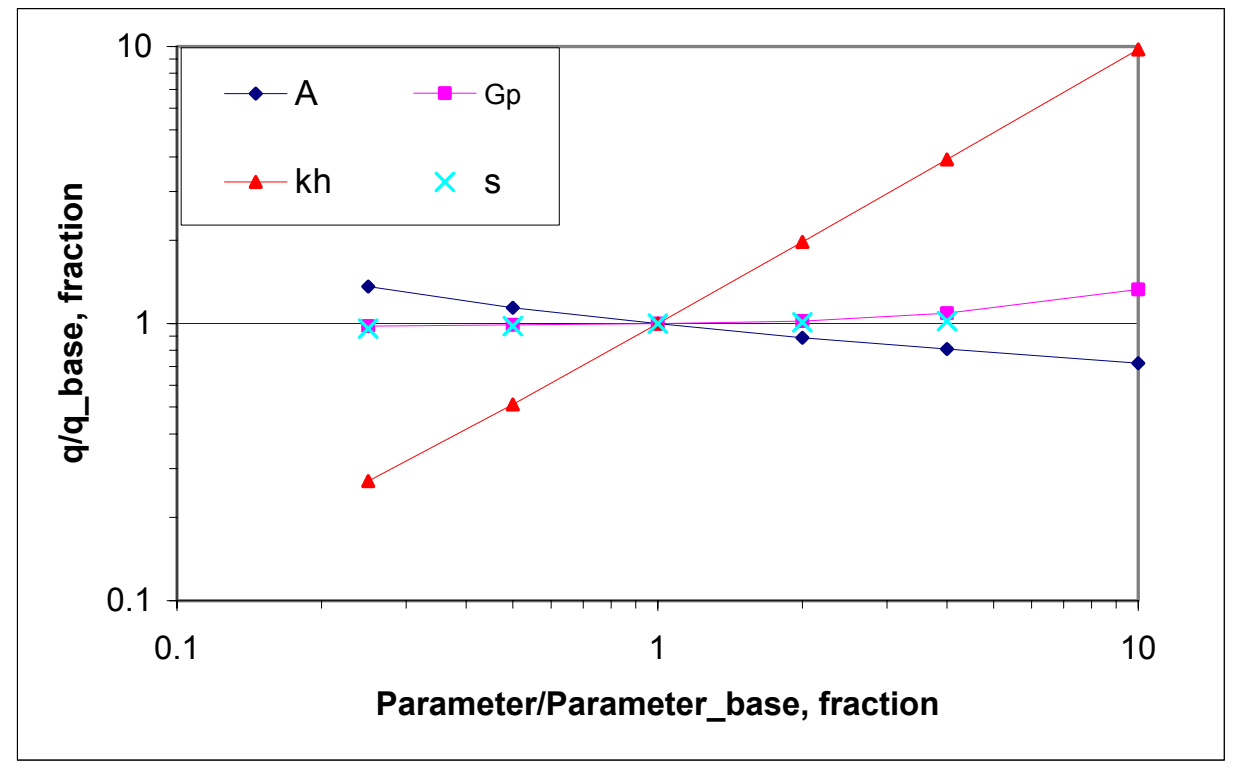

Fig. 2.7 Sensitivity study shows that $k h$ has greatest effect on $q$ calculation. 


\section{CHAPTER III}

\section{THE TESTING METHOD}

\subsection{Generation of the Test Data}

To create synthetic production data sets for testing and to investigate the effects of heterogeneity, we first generated permeability distributions for input to a gas-reservoir simulator. We created four cases, one homogeneous reservoir and three heterogeneous reservoirs. Then we calculated the infill-well performance with the moving window technology and compared it with the results from reservoir simulation. The simulation software packages we used are SABRE and Simulation Manager from Schlumberger.

Table 3.1 shows the parameters.

Table 3.1-Description of Four Cases

\begin{tabular}{|c|c|c|c|}
\hline Case & $\begin{array}{c}\text { Avg permeability } k, \\
\text { md }\end{array}$ & $\begin{array}{c}\text { STDEV of permeability } k, \\
\text { Md }\end{array}$ & $\begin{array}{c}C_{v} \text { of } \\
\text { permeability } k\end{array}$ \\
\hline 1 & 0.2 & 0 & 0 \\
\hline 2 & 0.2 & 0.06 & 0.33 \\
\hline 3 & 0.2 & 0.14 & 0.70 \\
\hline 4 & 0.2 & 0.24 & 1.25 \\
\hline
\end{tabular}

For the four cases, the average permeabilities are the same, $0.2 \mathrm{md}$. Case 1 is the homogeneous reservoir. For Cases 2 to 4, we used a log-normal distribution to generate a base random permeability field on a $54 \times 54 \times 1$ simulation grid. To obtain fields with different degrees of heterogeneity, we took running averages of the permeability distribution, using different averaging areas for each of Cases 2 to 4 . We used an $11 \times 11$ 
grid running average in Case 2 , a $5 \times 5$ running average in Case 3 , and a $3 \times 3$ grid running average in Case 4, respectively. Figs. 3.1 to 3.4 show the permeability distributions for Cases 1, 2, 3 and 4, respectively.
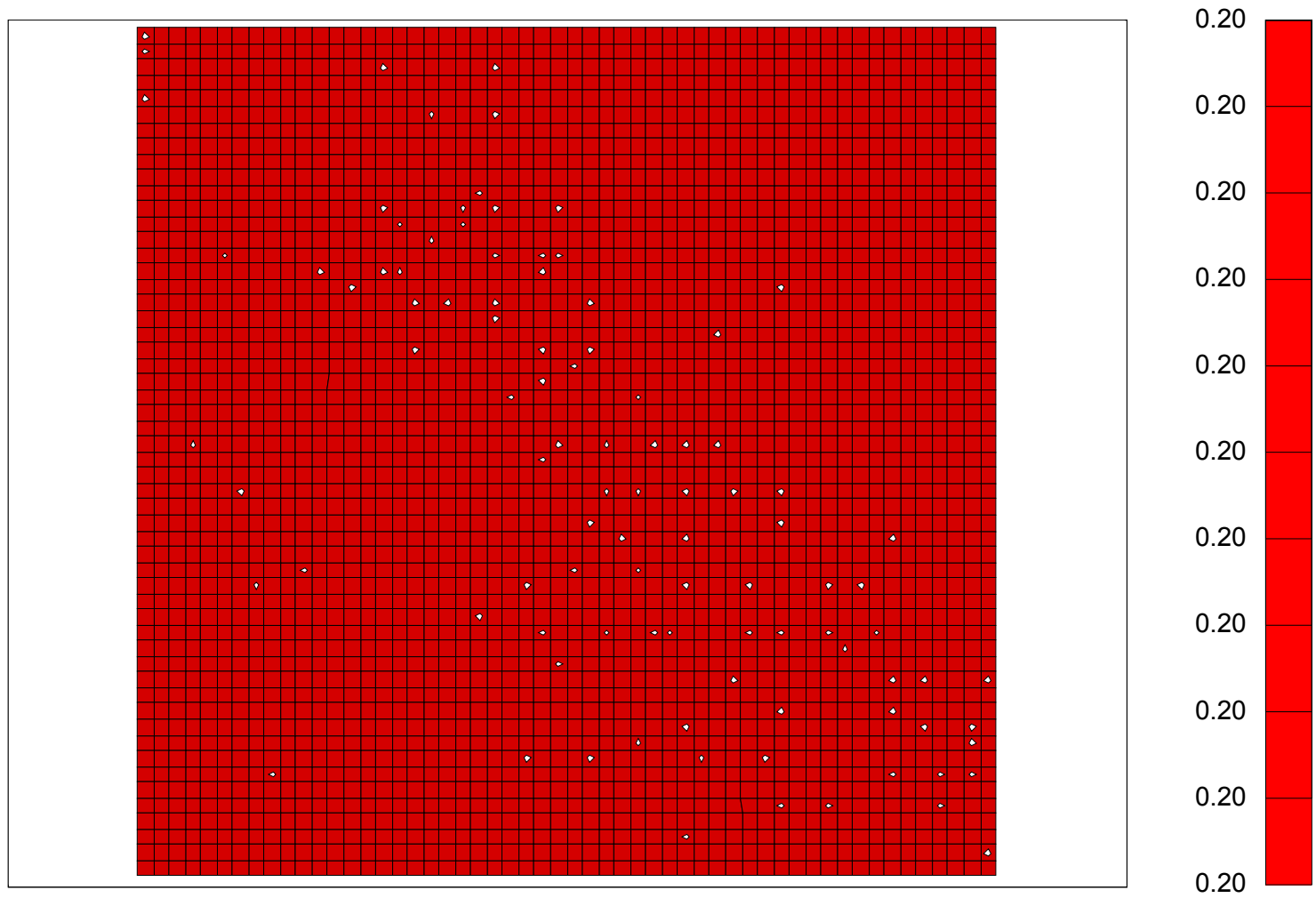

$12 / 16 / 196200: 00: 00 \quad 0.0000$ days

Fig. 3.1 Permeability $(k, \mathrm{md})$ distribution for Case 1. 

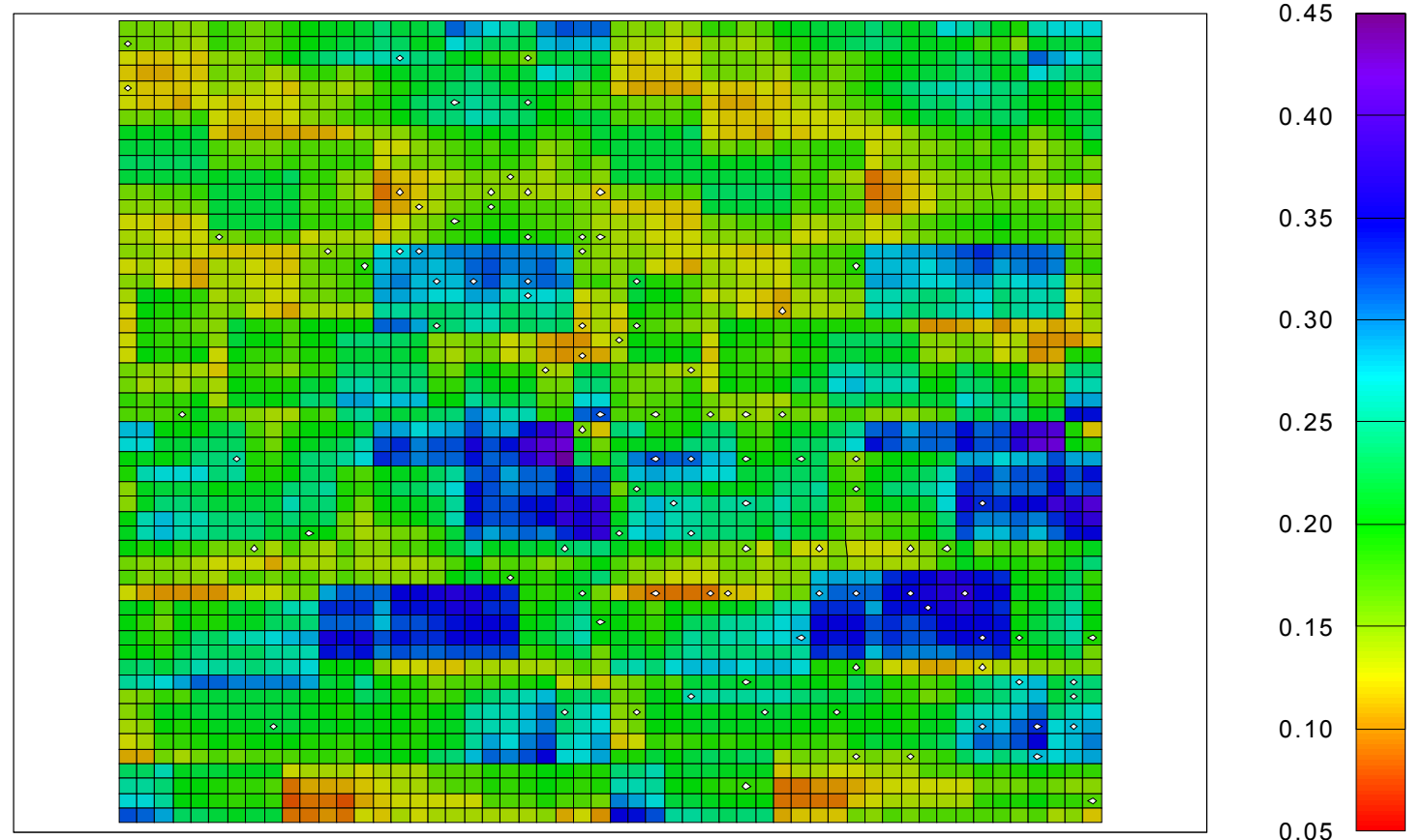

12/16/1962 00:00:00 0.0000 days

Fig. 3.2 Permeability ( $k, \mathrm{md})$ distribution for Case 2.
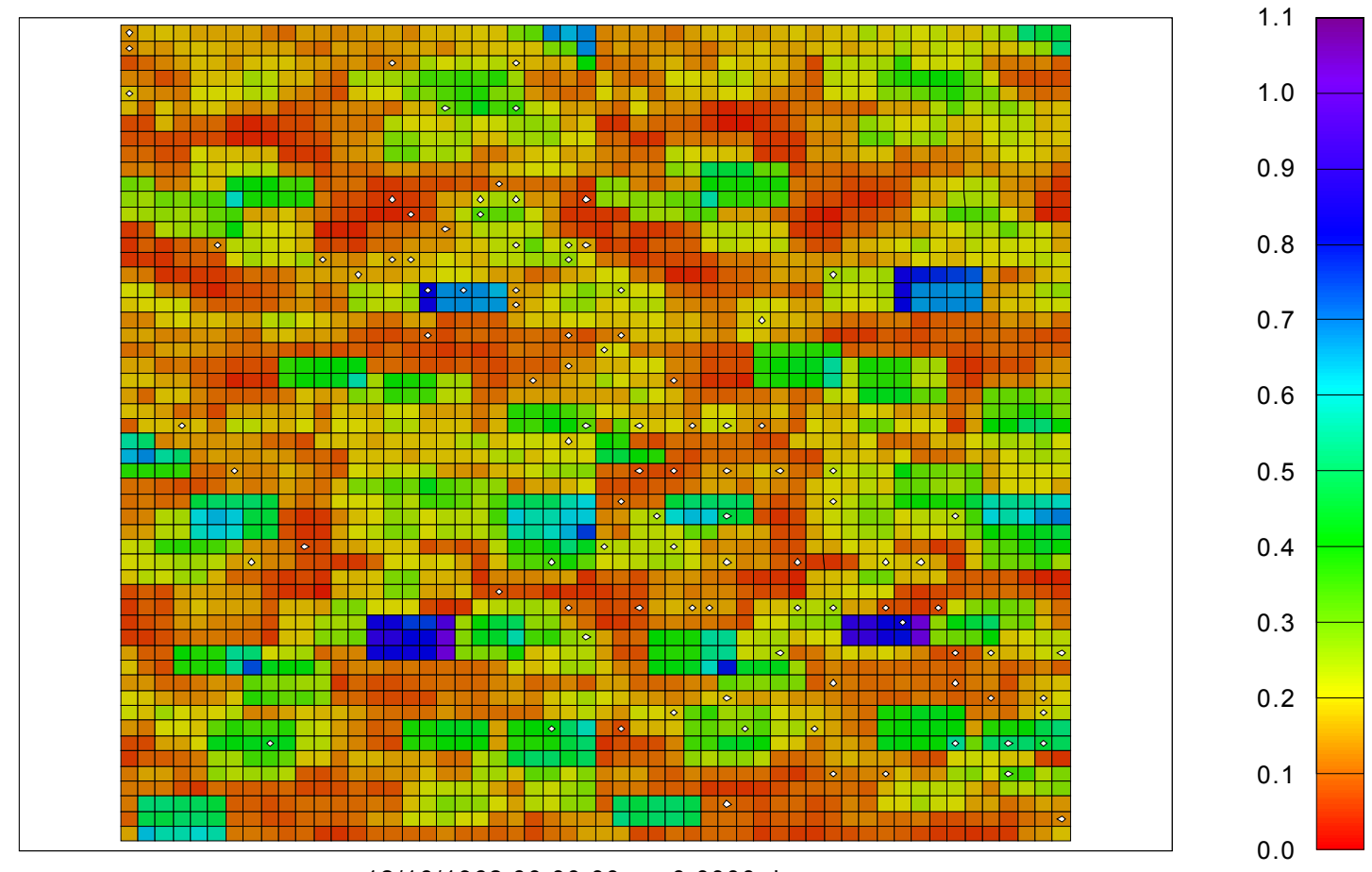

Fig. 3.3 Permeability $(k, \mathrm{md})$ distribution for Case 3. 


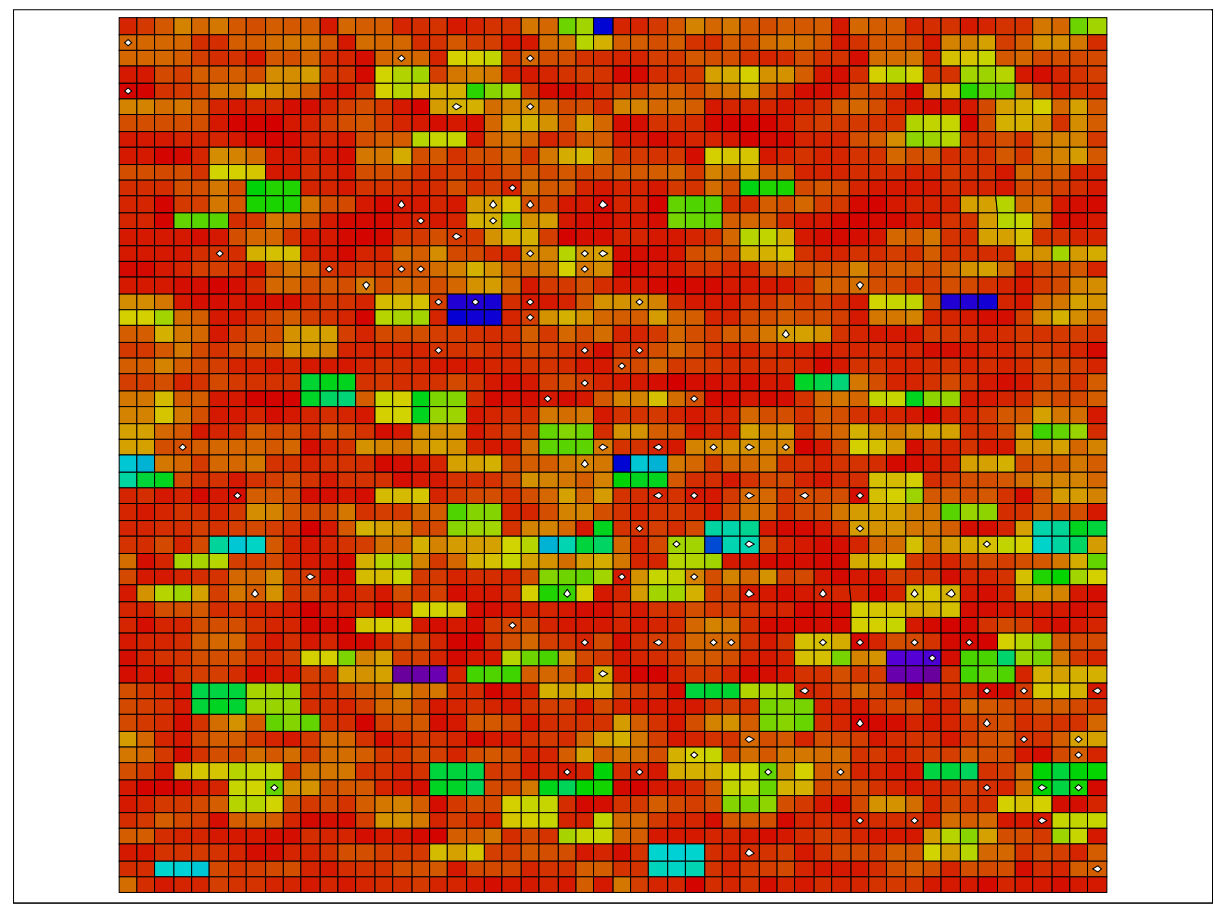

$12 / 16 / 196200: 00: 00 \quad 0.0000$ days

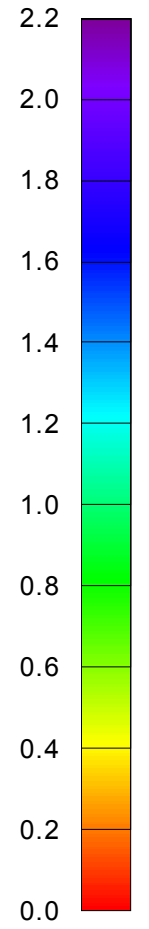

Fig. 3.4 Permeability $(k, \mathrm{md})$ distribution for Case 4.

From these four figures, we see that the reservoir becomes more heterogeneous as the case number increases. This is also indicated by the increase in standard deviation (STDEV) and coefficient of variation $\left(C_{v}\right)$ with case number (Table 3.1).

The locations for the 100 wells simulated in the study area are also shown in Figs. 3.1 to 3.4. We used realistic distributions of the current well spacing (Fig. 3.5) from a Canadian gas field. The average current spacing of the 100 wells is 535 acres. The production starting dates are also the real first starting production dates for the 100 wells (Fig. 3.6). The earliest production date of the 100 wells is 19 December, 1962 , and latest production date is 17 August, 2000. 


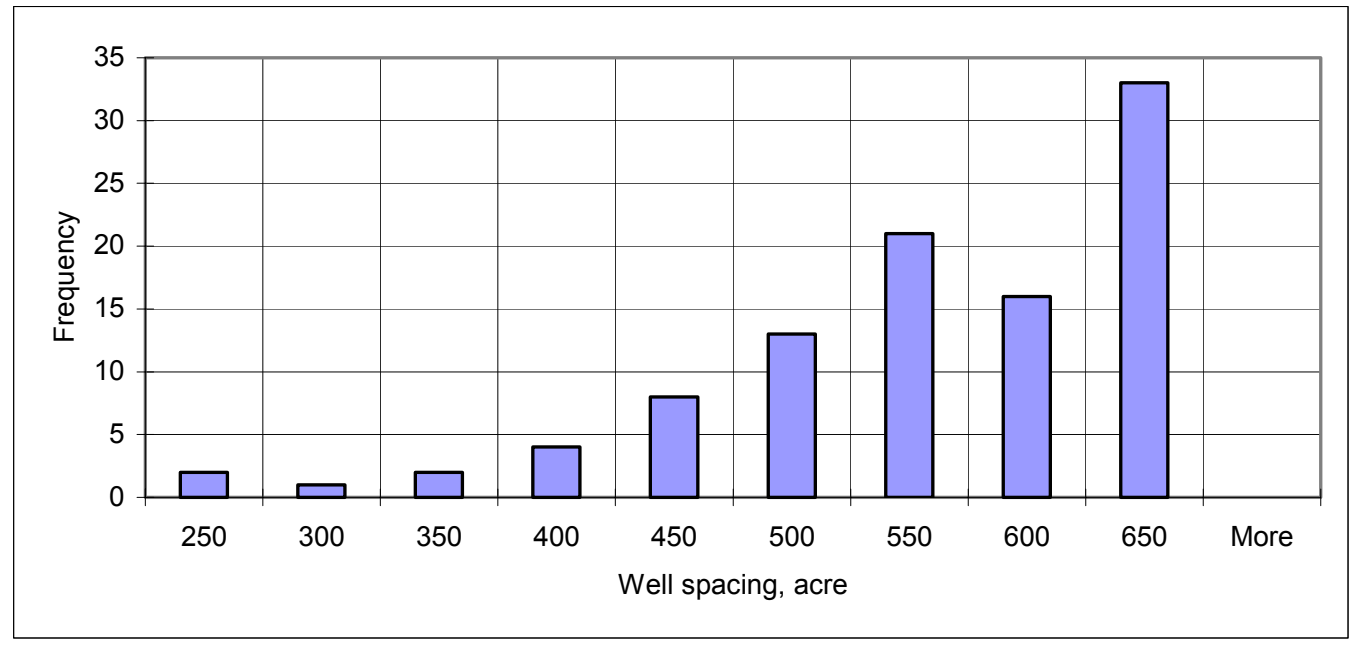

Fig. 3.5 Histogram of current well spacing for the 100-well cases.

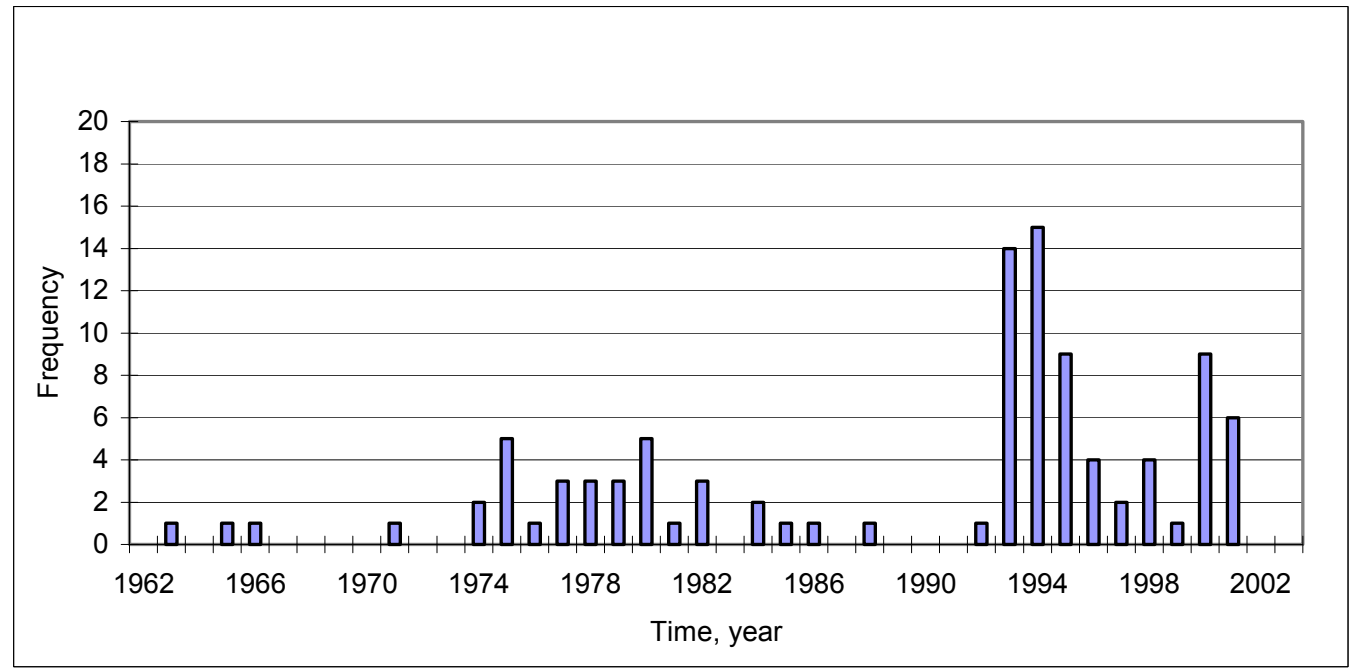

Fig. 3.6 Histogram of date of first production for the 100-well cases.

The well numbers and locations of existing wells, as well as the remaining parameters listed below, were the same for all four cases.

Porosity: $12 \%$.

Initial reservoir pressure: 1,100 psia.

Flowing bottomhole pressure: 250 psia. 
Well skin factor: -3 .

Well bore radius: $0.3 \mathrm{ft}$.

Water saturation: $40 \%$.

We next ran the simulator to generate the 100 wells' production history that was analyzed using the moving window technique to estimate infill well performance. The reservoir history included wells starting at different dates over a period of approximately 40 years to represent several rounds of infill drilling. The simulations were run through 17 August, 2001. The simulated production data were then analyzed using the moving window technique to determine infill well performance. We used a search area of 3000 acre for the moving window analysis.

\subsection{Validation of VBY Estimation}

The prediction of VBY is a very important step in the moving window technology, since VBY serves as a proxy for $k h$, or reservoir quality, in the 4D regression model. The parameter sensitivity study (Chapter 2.3) showed that the accuracy of the moving window method is most sensitive to the estimation of $k h$. Thus, our first test was to determine how well VBY was being predicted by the $2 \mathrm{D}$ regression of BY vs. well start date in each moving window.

To determine the true VBY for each existing well, we made a separate simulation run for each well in which we produced only that well for a year. Figs. 3.7 to 3.10 show the comparison of VBY from the 2D regression to the true value determined from simulation. The solid black lines are the 1:1 line in these figures. Figs. 3.11 to 3.14 show how VBY from the 2D regression compares to $k h$ for the four cases, and the solid lines in these figures are the best-fit lines. 


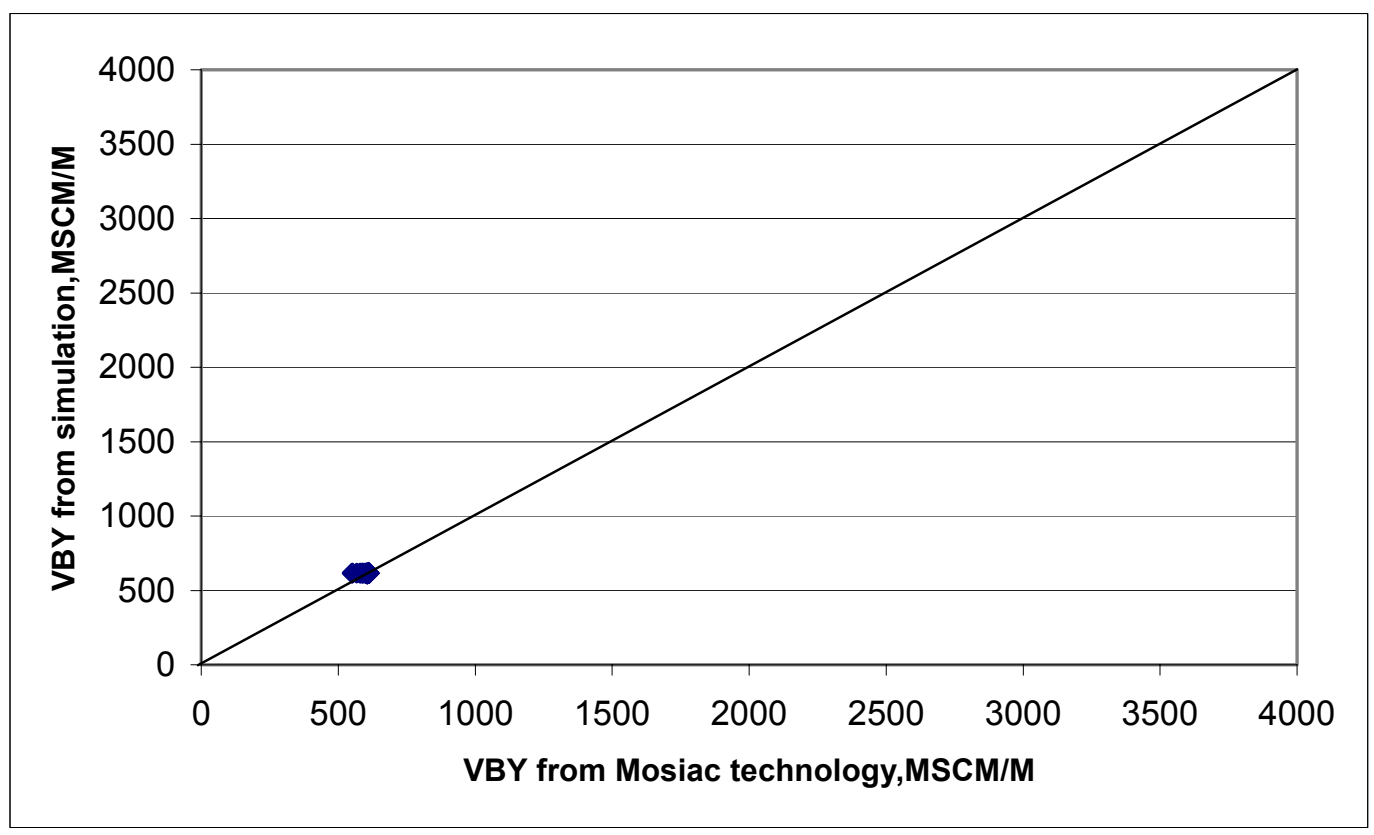

Fig. 3.7 Comparison of VBY from simulation and Mosaic technology for Case 1. VBY from Mosaic technology shows little deviation from simulation for Case 1.

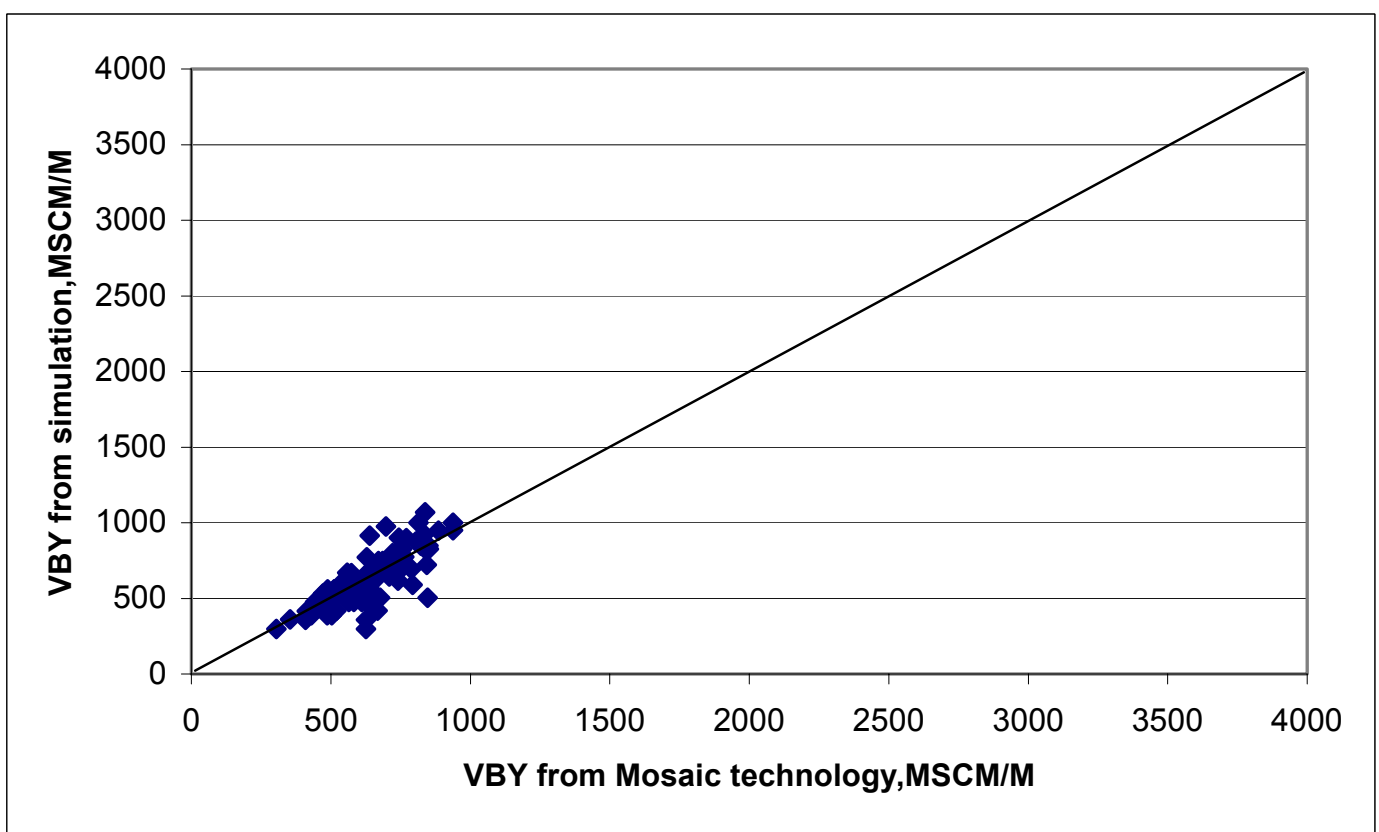

Fig. 3.8 Comparison of VBY from simulation and Mosaic technology for Case 2. VBY from Mosaic technology correlates well with simulation for Case 2. 


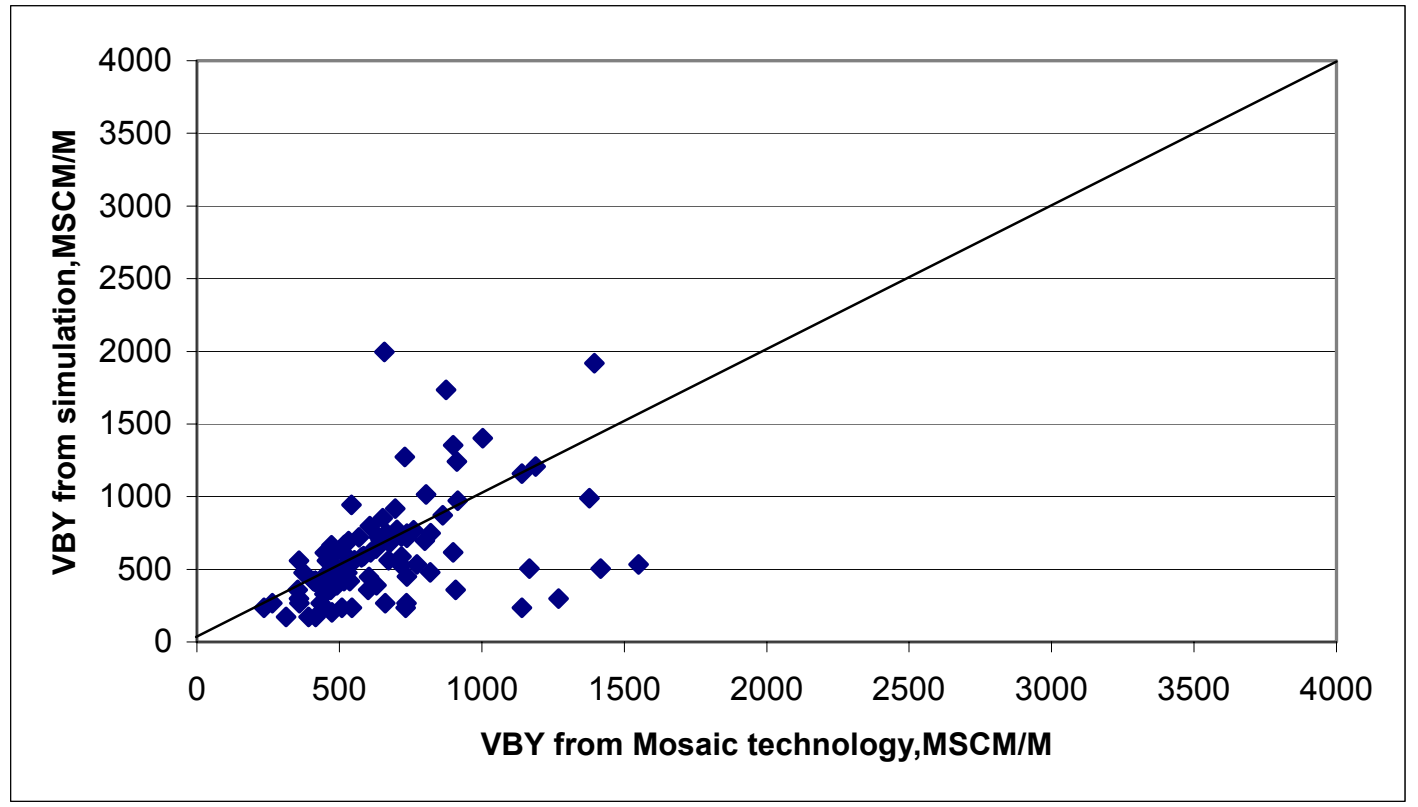

Fig. 3.9 Increased heterogeneity in Case 3 introduces scatter between VBY from Mosaic technology and simulation.

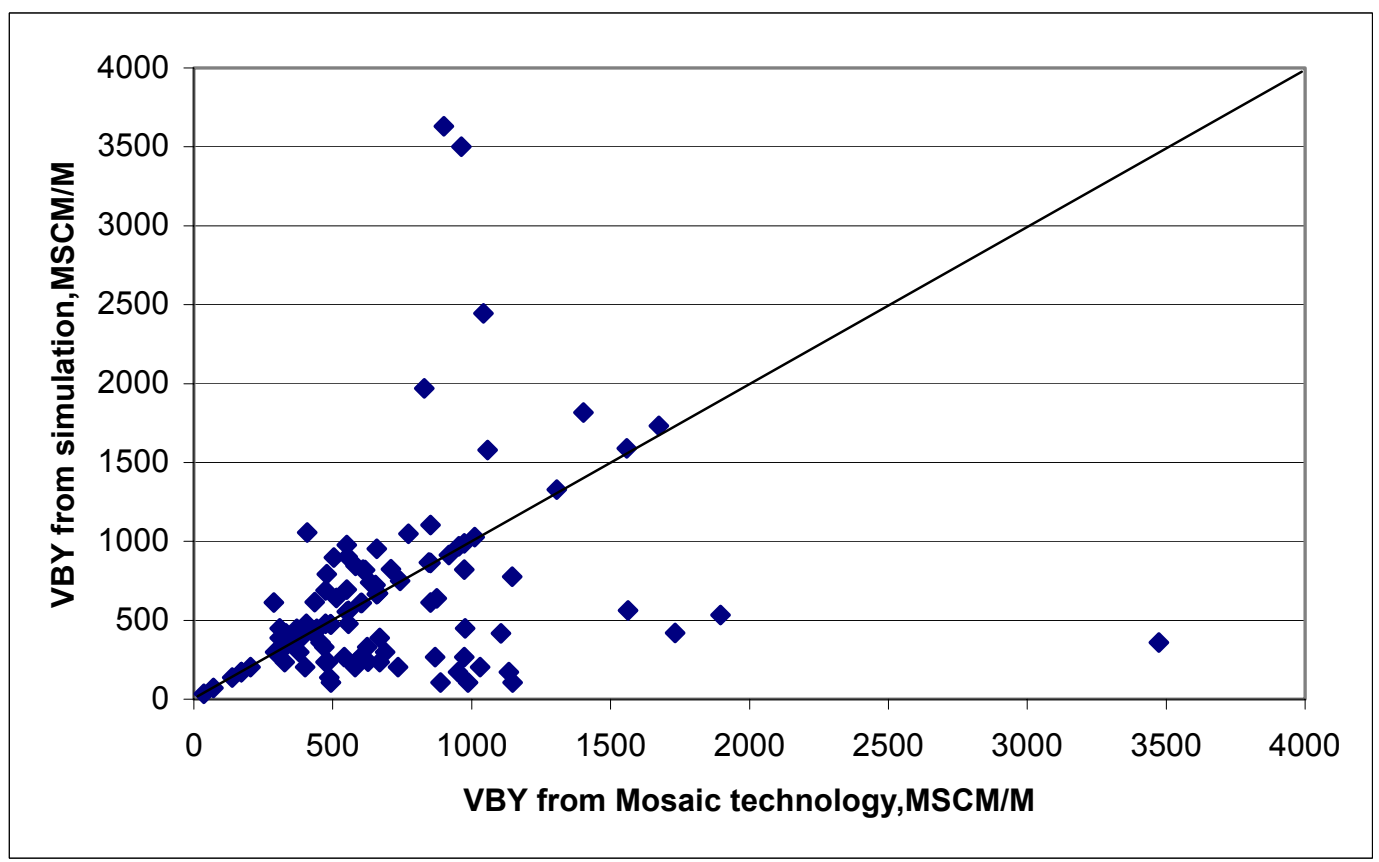

Fig. 3.10 High heterogeneity of Case 4 results in great scatter in VBY between Mosaic technology and simulation. 


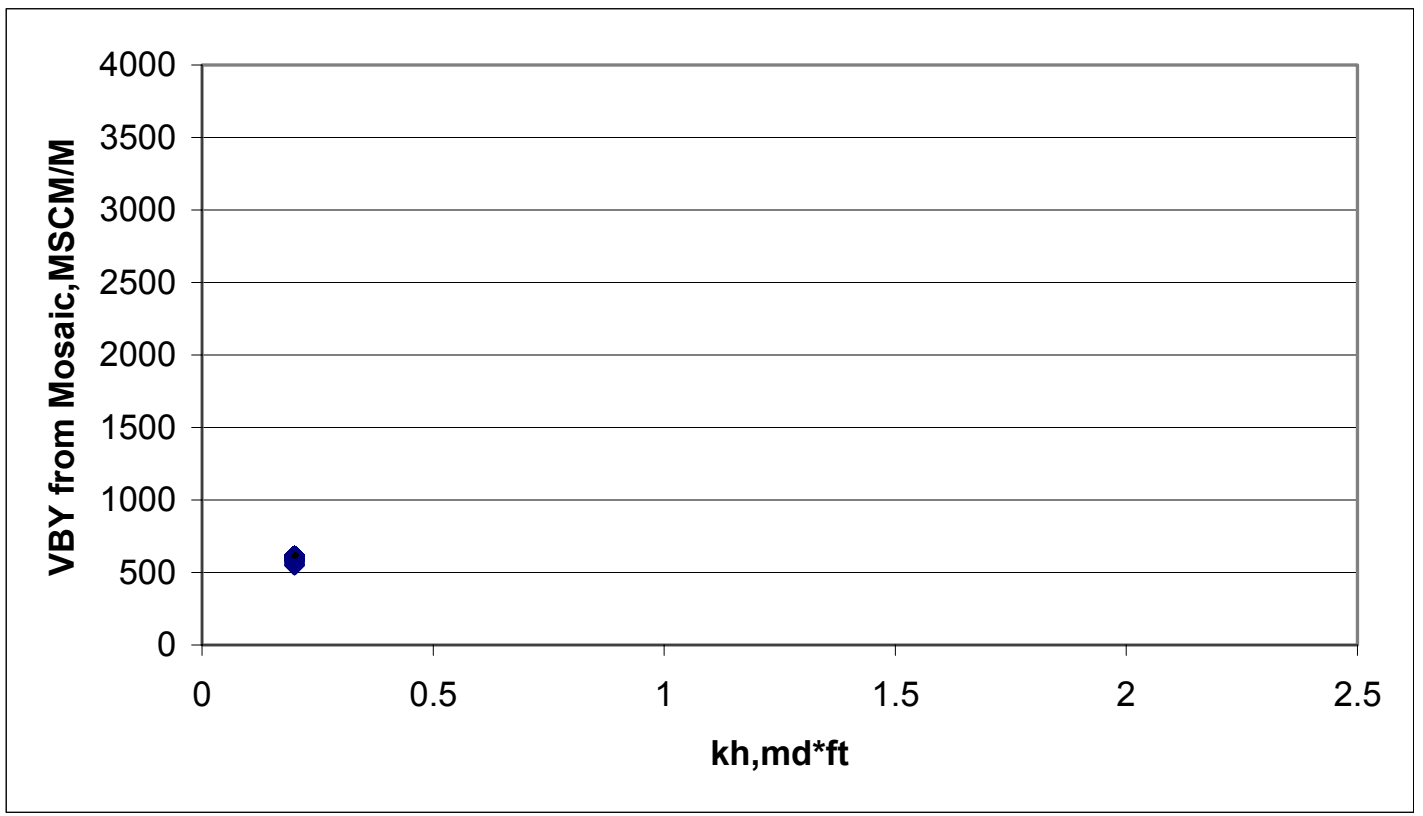

Fig. 3.11 VBY serves well as a proxy for $k h$ for Case 1 .

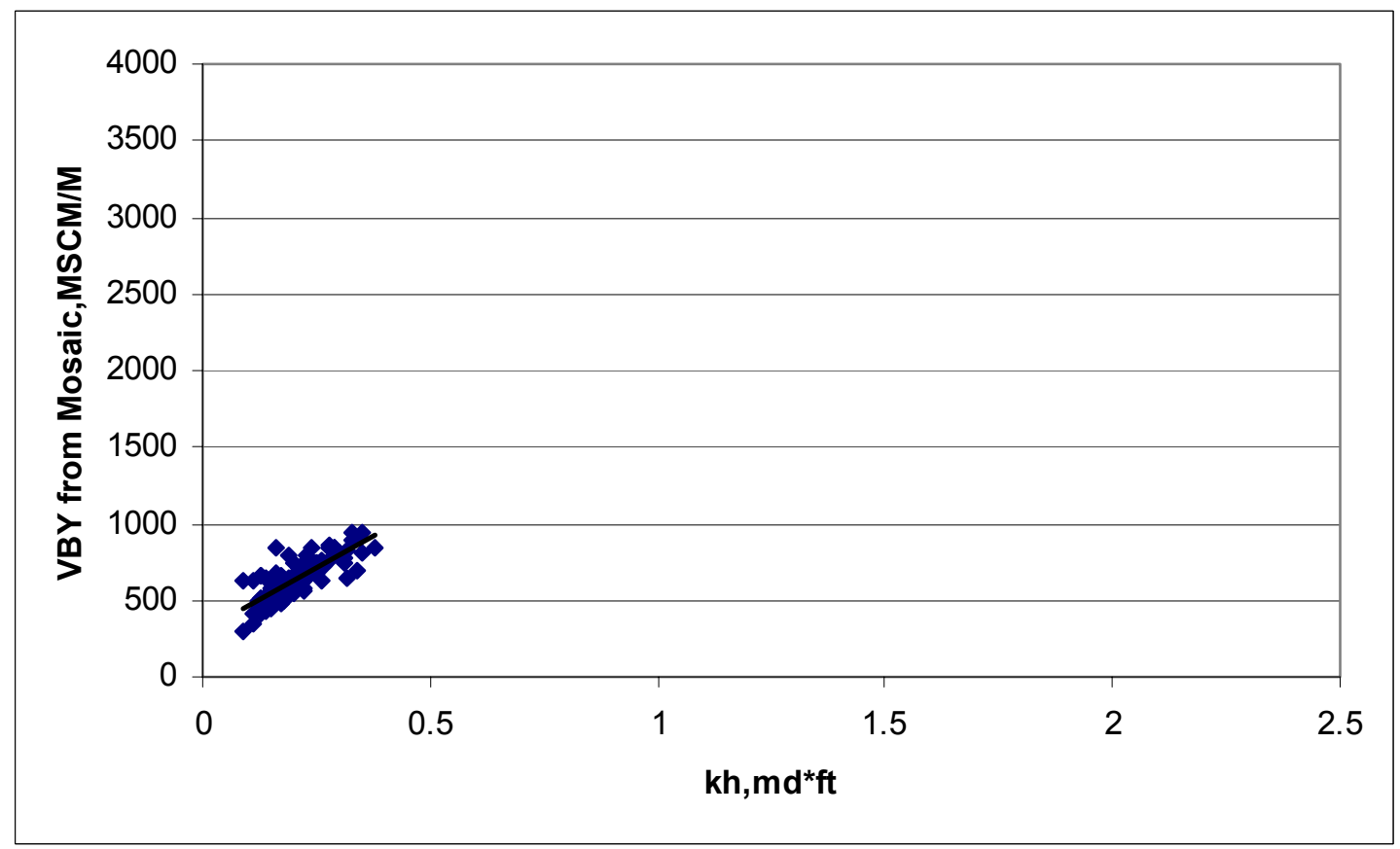

Fig. 3.12 VBY serves well as a proxy for $k h$ for Case 2. 


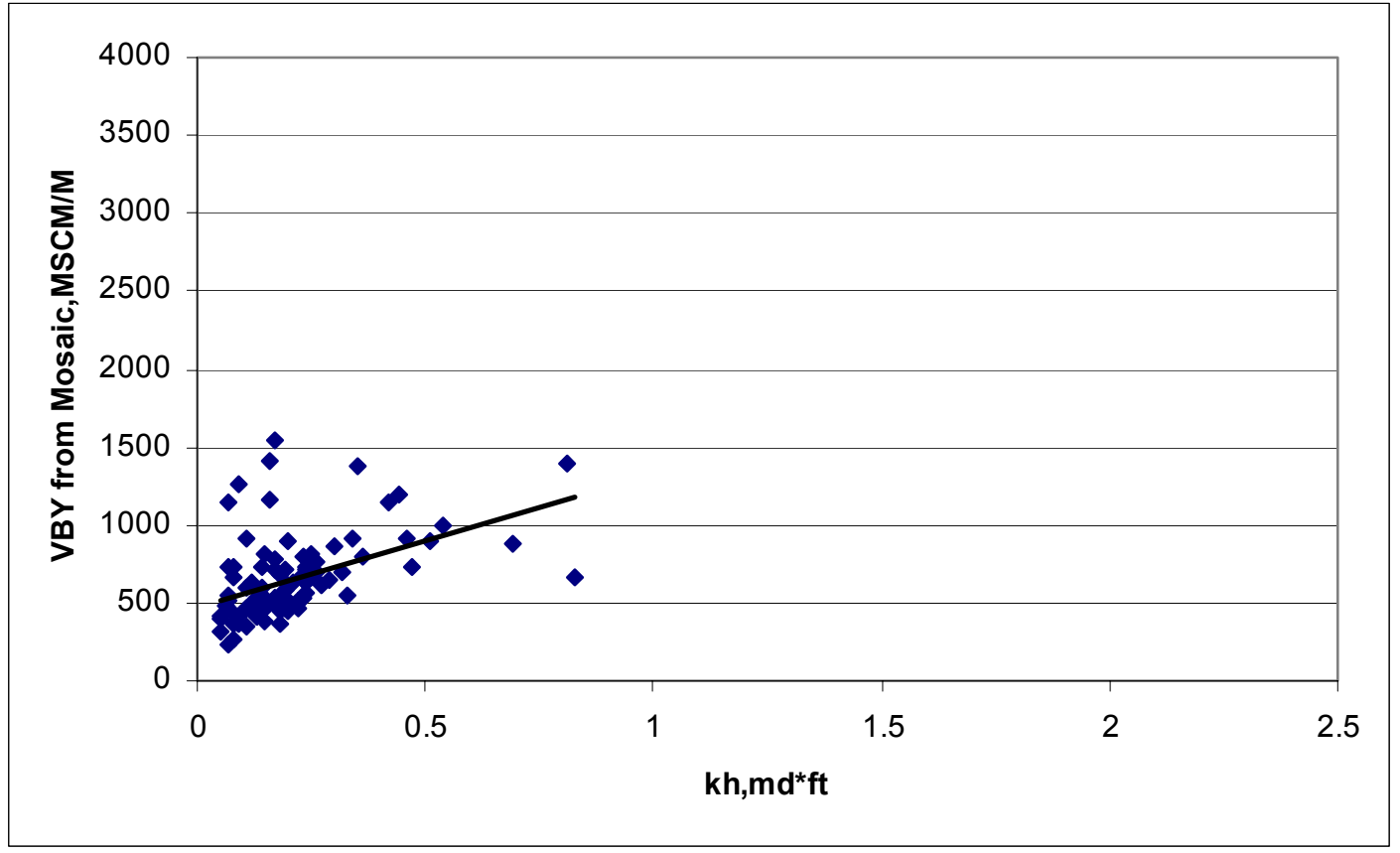

Fig. 3.13 Increased heterogeneity reduces value of VBY as a proxy for $k h$ for Case 3 .

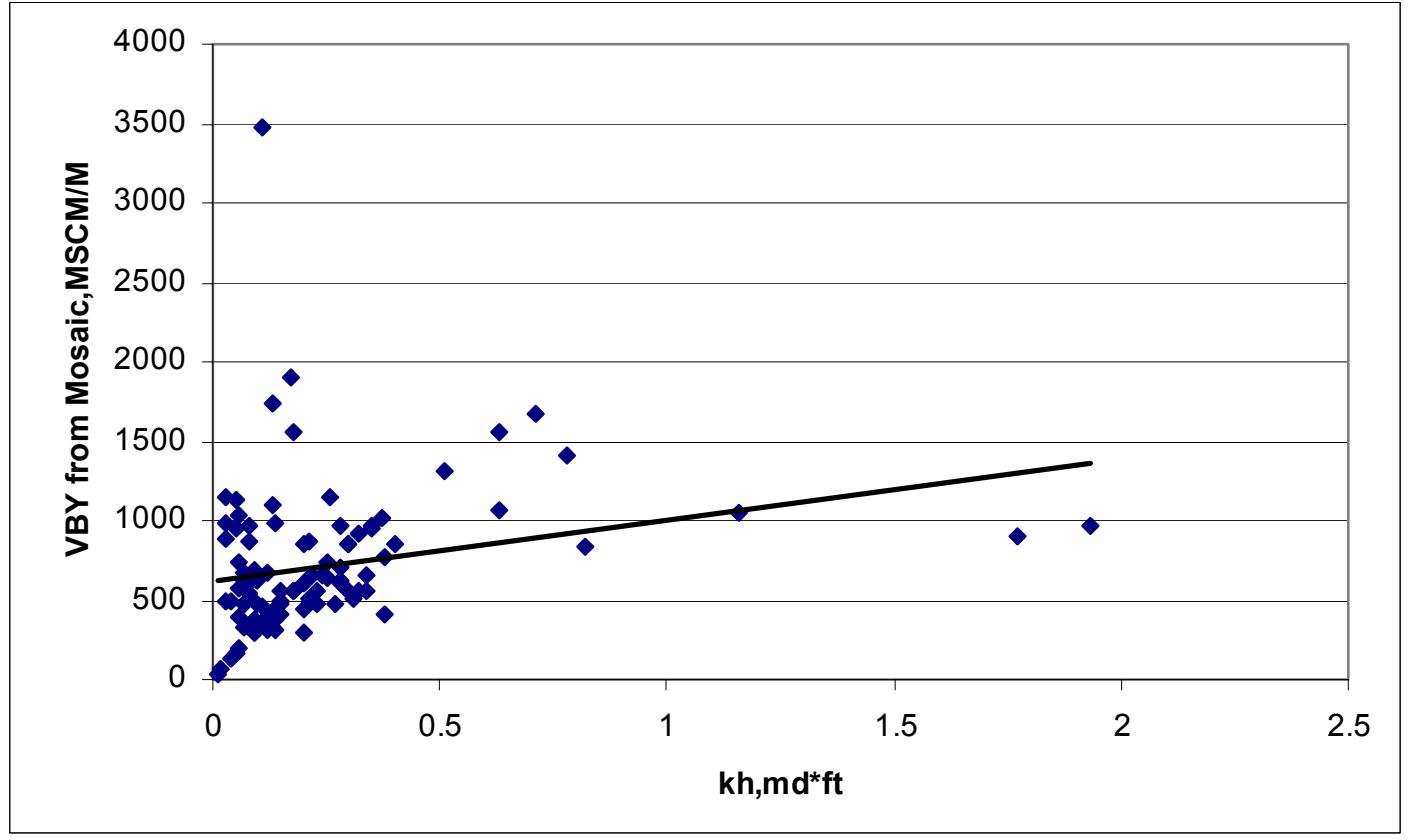

Fig. 3.14 High heterogeneity of Case 4 severely reduces effectiveness of VBY as a proxy for $k h$. 
Figs. 3.7 to 3.10 indicate that the estimation of VBY is best for homogeneous reservoirs, while accuracy decreases as heterogeneity increases. The data points become more scattered as the case number increases.

Figs. 3.11 to 3.14 indicate good correlation between VBY and $k h$ for Case 1 and Case 2. But for Case 3 and Case 4, the data points become more widely scattered. The black lines in Fig 3.12 to 3.14 are the least-square fit lines calculated by Excel.

Table 3.2 summarizes the comparison of VBY determined from Mosaic and simulation. Even though the data points are widely scattered in Case 4, the most heterogeneous of the four cases, the average relative error is only about $10.5 \%$. The results demonstrate that VBY serves as a reasonable proxy for $k h$, and that VBY can be estimated reasonably well from the $2 \mathrm{D}$ regression.

Table 3.2 -VBY Comparisons From Mosaic and Simulation Methods

\begin{tabular}{|c|c|c|c|}
\hline Case & $\begin{array}{c}\text { Avg VBY from } \\
\text { Mosaic, } \\
\text { MSCM/M }\end{array}$ & $\begin{array}{c}\text { Avg VBY from } \\
\text { simulation, } \\
\text { MSCM/M }\end{array}$ & $\begin{array}{c}\text { Relative Error in } \\
\text { Avg VBY, } \\
\%\end{array}$ \\
\hline 1 & 607 & 615 & -1.3 \\
\hline 2 & 636 & 625 & 1.8 \\
\hline 3 & 647 & 607 & 6.6 \\
\hline 4 & 714 & 646 & 10.5 \\
\hline
\end{tabular}

From Table 3.1 and Figs. 3.1 to 3.4, we know that the reservoirs become more heterogeneous as the case number increases. In Cases 1 and 2, the reservoirs are relatively homogenous and the reservoir properties do not change much within each 3,000-acre (about 36 grid cells) searching domain. In Cases 3 and 4, the reservoir properties change very rapidly within each search domain. But the Mosaic technology 
still assumes the reservoir is homogenous in each searching domain. This is why the data become more scattered in Case 3 and Case 4. The effects of heterogeneity will be discussed further in Section 4.1.

\subsection{The Testing Procedure}

The next step was to determine how well the moving window technique predicts infill well performance. To do this it was necessary to determine the true infill well performance from simulation. We placed a new well in the first grid block and made a 1 -year projection, from the end of history, to determine the production to be gained by a new well in this location. We then repeated this for each grid block in the system, thus generating a map, or distribution, of the additional production to be attributed to one new well at all the possible locations in the reservoir. For each run, we calculated both the new well's 1-year cumulative production and the corresponding field's 1-year incremental production, which accounts for reserves lost through interference with existing wells.

Fig. 3.15 compares new-well 1-year cumulative production vs. incremental field 1 -year production. The plot contains 2,916 data points, one for each cell of the $54 \times 54$ grid. The agreement between one well's cumulative production and its corresponding incremental field production indicates that this well's performance does not influence its neighboring wells. From this plot, we find that the new-well 1-year cumulative production is essentially equal to the corresponding incremental field production for most of the wells, indicating that most of the wells in the field do not have a significant effect on one another. This result holds for the permeabilities and well spacings used in these test cases; it will not necessarily apply to all reservoirs. 


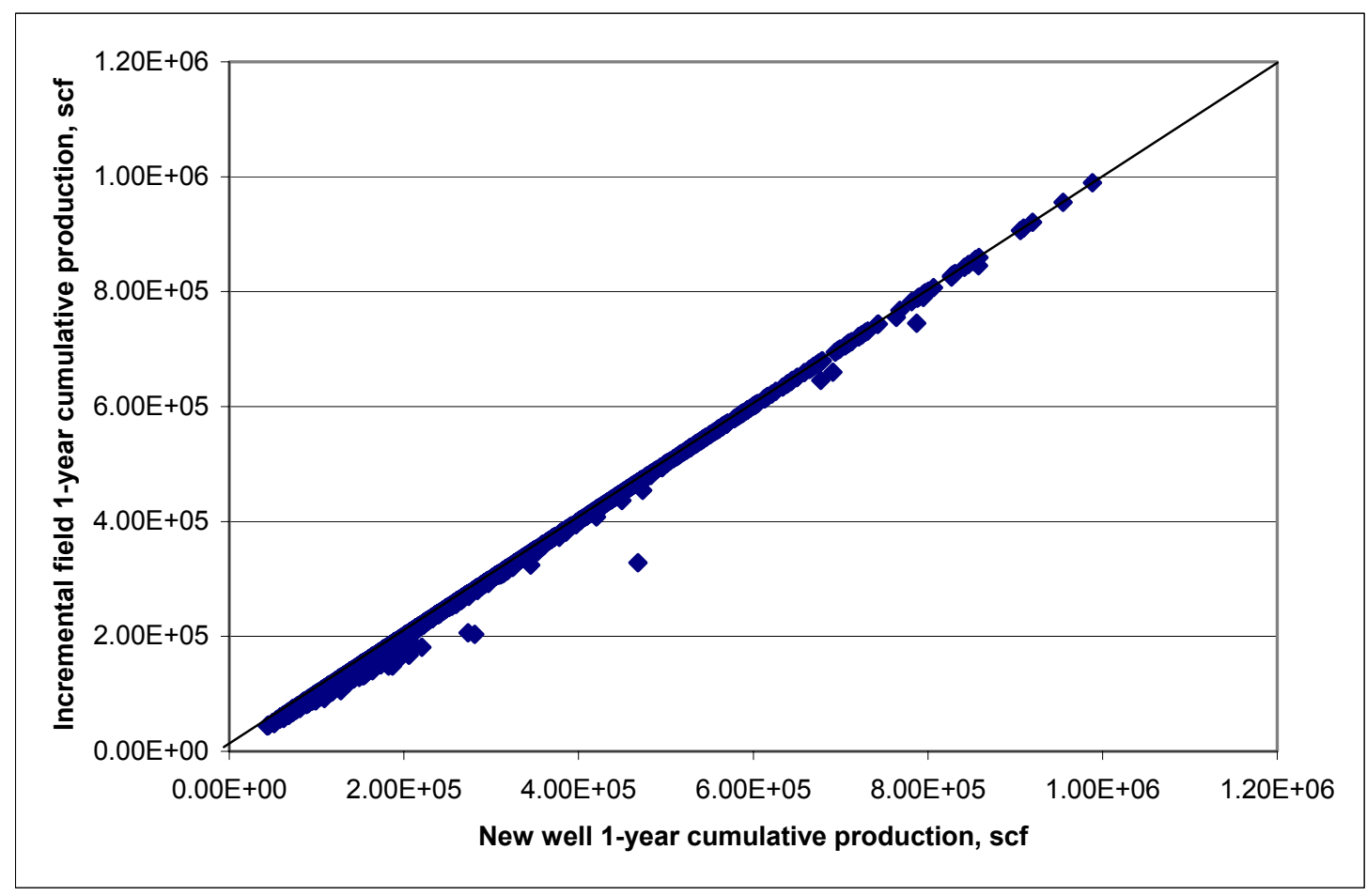

Fig. 3.15 New-well 1-year cumulative production corresponds well with incremental field 1-year production.

While the incremental field 1-year cumulative production is probably a better measure of infill performance, we used the new-well 1-year cumulative production in subsequent work, primarily because this is the quantity that is calculated by the moving window technique and our interest is in comparing moving window results to results from simulation.

Mosaic results must be at the same resolution as simulation results for comparison. The infill-well performance estimates from simulation are on a cell basis, while Mosaic results are on a well basis. Therefore, the simulation results must be converted to a well basis. To do this we first determined, for each well, the region consisting of all simulation cells closer to that well than to any other well. 
For unbounded wells, we limited the search radius of the circular region to correspond approximately to the maximum search area used in the Mosaic analysis, 3,000 acres. Fig. 3.16 illustrates the calculated well regions.
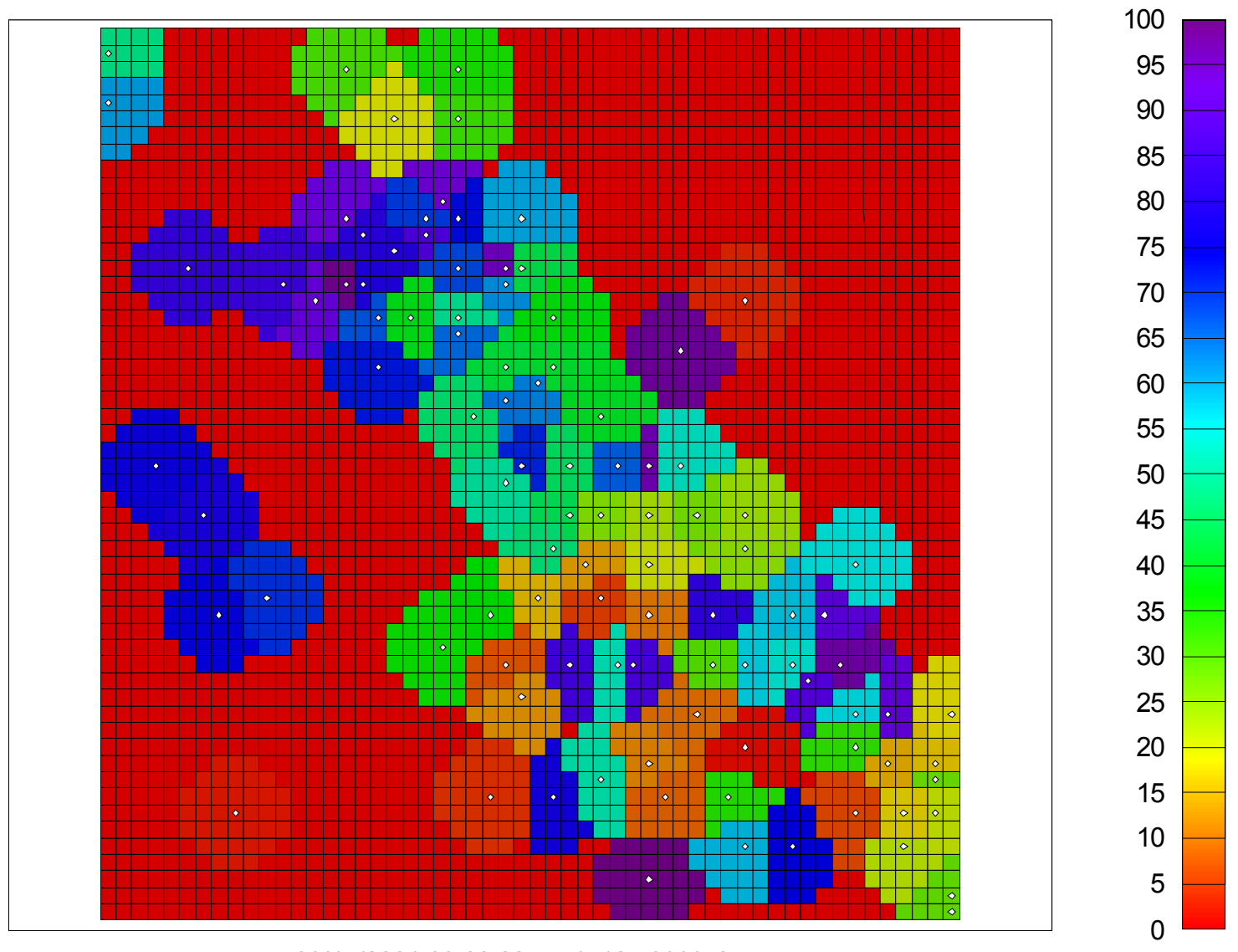

Fig. 3.16 Simulation well regions.

The next step was to calculate a new-well cumulative production for each region from the individual cell values. We considered using either the maximum new well BY in each well's simulation region (Max_New_Well_BY) or the arithmetic average of new well BY over all the cells in each well's simulation region (Avg_New_Well_BY). 
Fig. 3.17 shows the relationship between Max_New_Well_BY and Avg_New_Well_BY for Case 3. Avg_New_Well_BY appears to be a better standard for benchmarking the moving window results, since

(1) The moving window technique tends to average results within each domain, and

(2) Operators will not have detailed knowledge of permeability distributions required to determine the location that maximizes production within a local domain.

Thus, Avg_New_Well_BY was used as the standard for comparison henceforth and we take it as the infill BY calculated from the simulation.

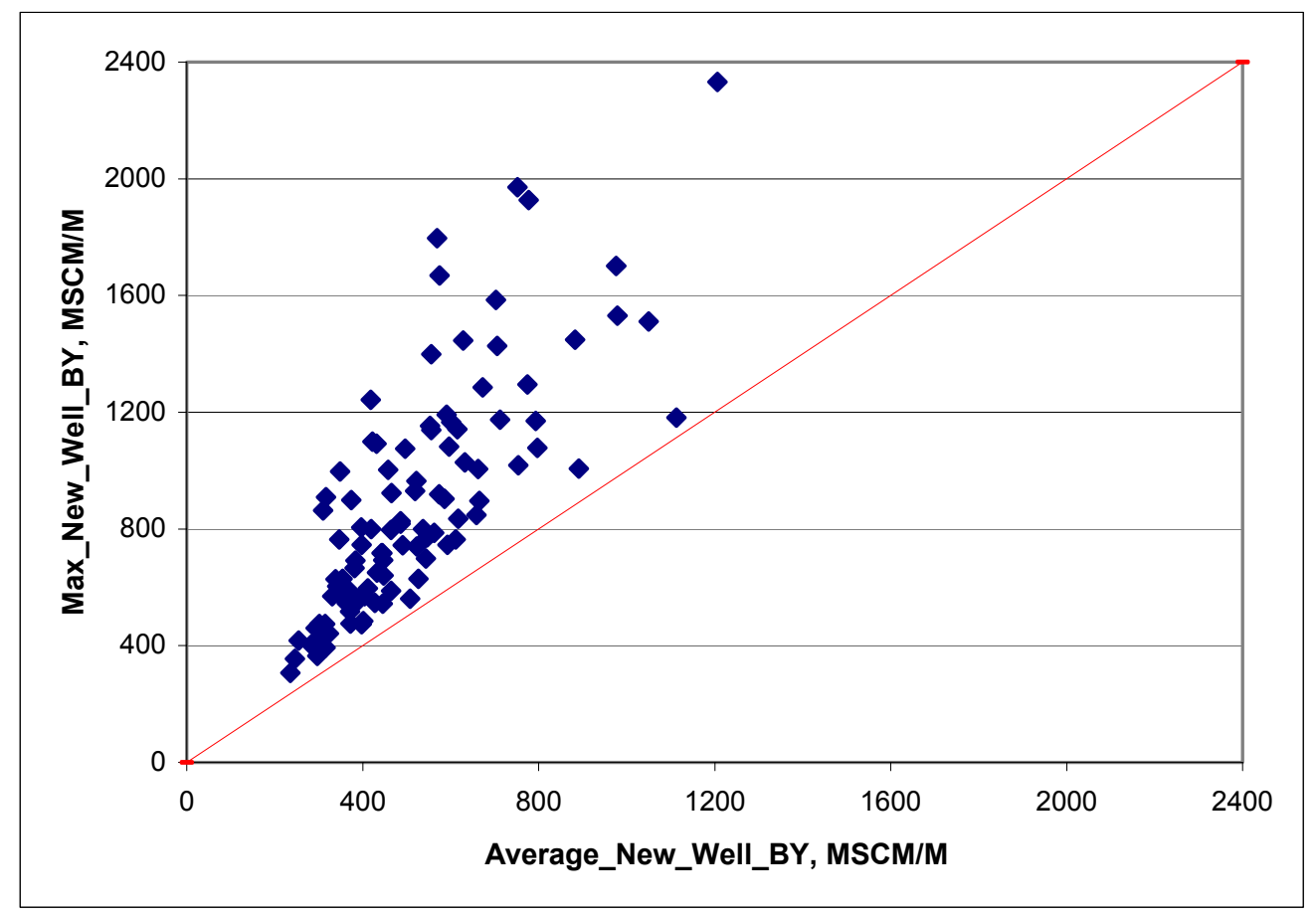

Fig. 3.17 Max_New_Well_BY vs. Avg_New_Well_BY for Case 3. 
Figs. 3.18 and 3.19 show the comparisons of infill BY from Mosaic technology to Max_New_Well_BY and Avg_New_Well_BY for Case 3, respectively. The $+/-30 \%$ and $+/-50 \%$ lines on the plots indicate the relative accuracy of the moving domain estimates. Examination of Figs. 3.18 and 3.19 validates the choice of Avg_New_Well_BY as the standard for benchmarking the moving window technique.

Here we show only the comparison plots for Case 3. Comparison plots for Case 1, Case 2, and Case 4 yield similar conclusions.

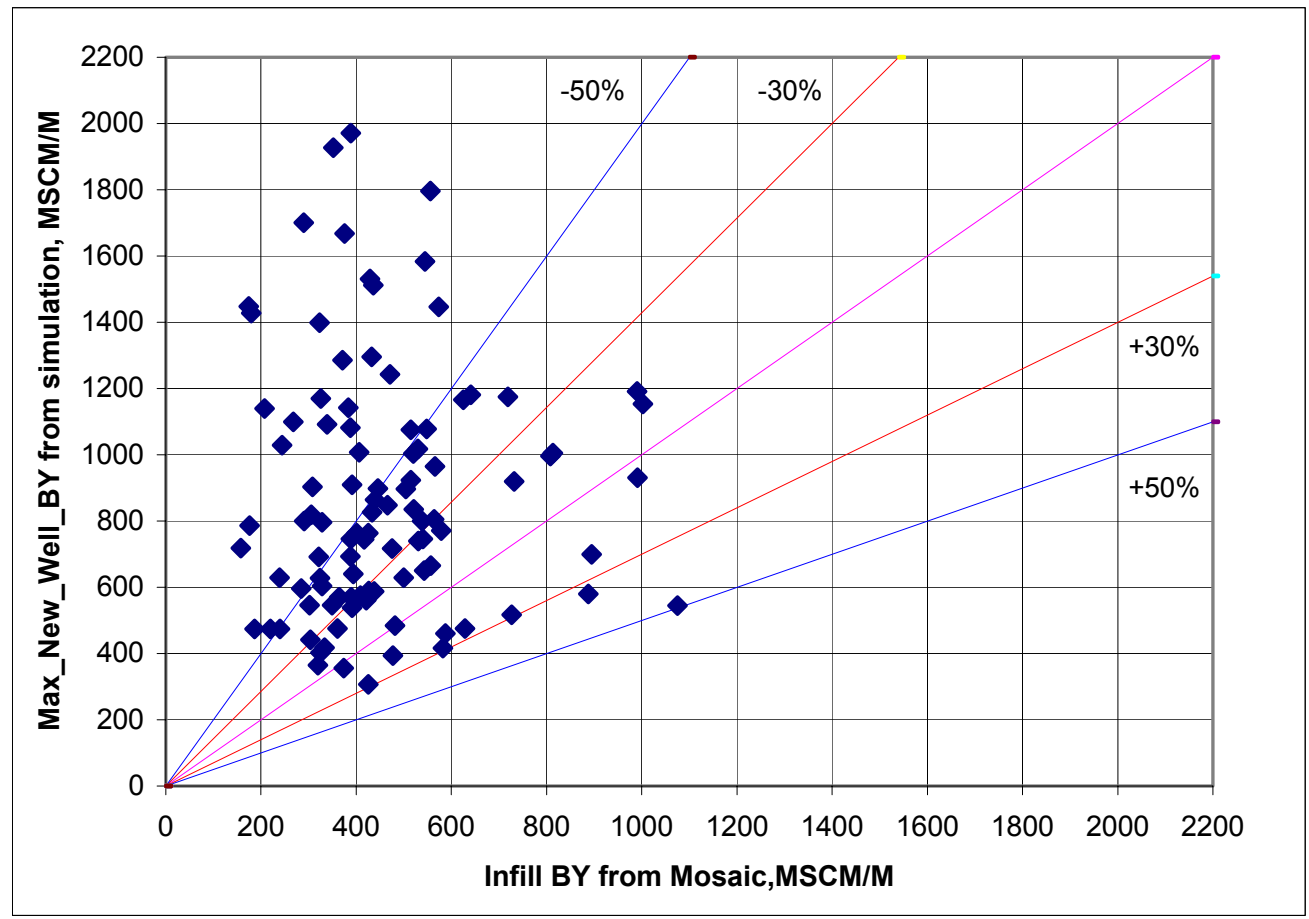

Fig. 3.18 Infill BY vs. Max_New_Well_BY for Case 3. 


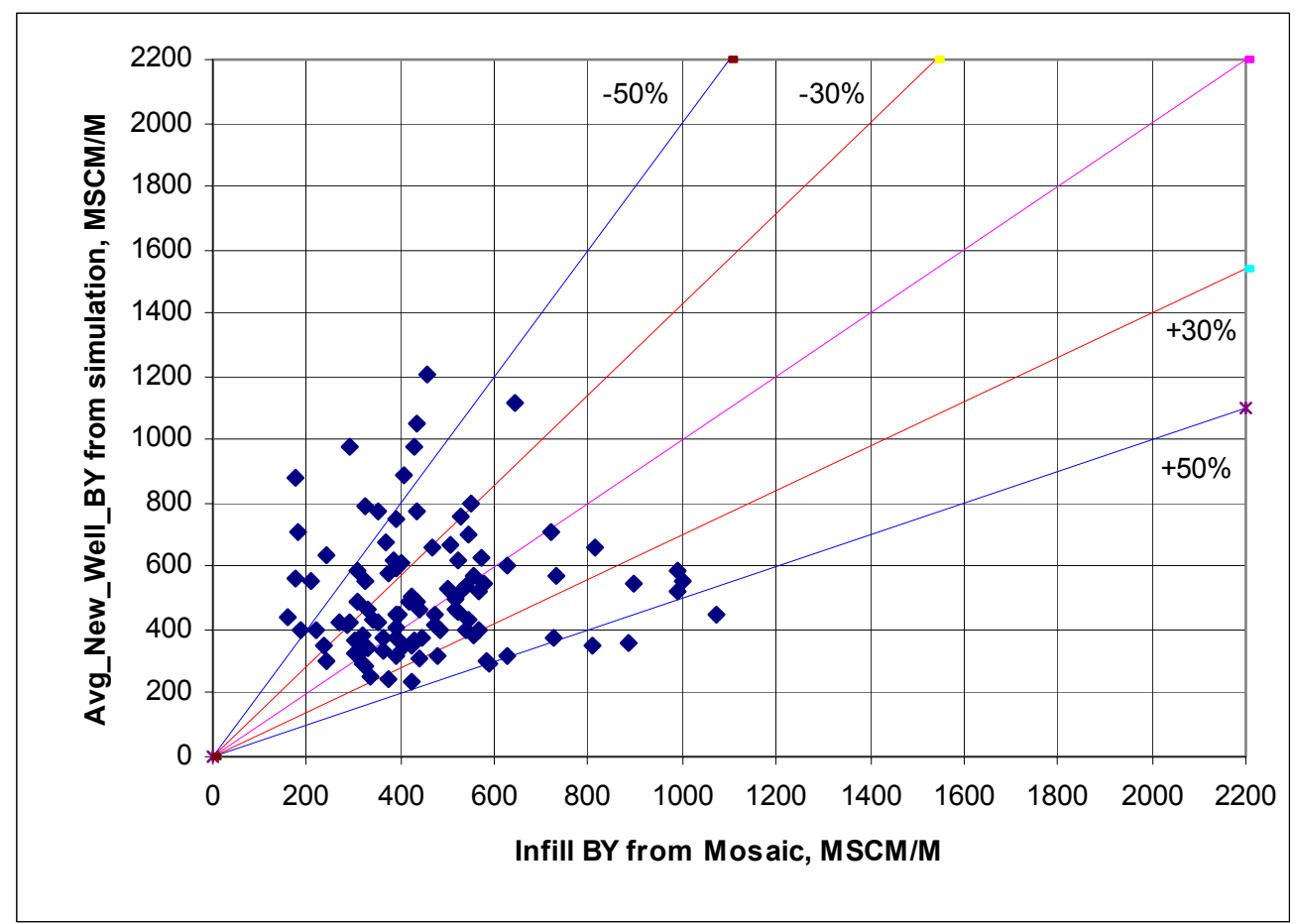

Fig. 3.19 Infill BY vs. Avg_New_Well_BY for Case 3.

\subsection{Depletion Factor}

To ensure valid comparisons of infill well performance prediction between cases, we needed to verify that all four cases were at a similar stage of depletion at the latest production date. To quantify the degree of depletion in our study area we define a depletion factor (DF):

$$
D F=1-\frac{\text { Average of } B Y \text { from NewWells }}{\text { Average of VBY from Existing Wells }}
$$

A depletion factor of 0.0 means there is no depletion in the study area. From the definition of DF we know that the greater the DF, the greater the depletion the reservoir has experienced. 
The depletion factor calculated for each case is listed in Table 3.3. The depletion factors range from $10 \%$ to $19 \%$ across the four cases. These results indicate that the four cases were at a similar stage of depletion and they are comparable.

Table 3.3-Depletion Factors of Four Cases

\begin{tabular}{|c|c|}
\hline Case & $\begin{array}{c}\text { Depletion Factor, } \\
\%\end{array}$ \\
\hline 1 & 12 \\
\hline 2 & 10 \\
\hline 3 & 15 \\
\hline 4 & 19 \\
\hline
\end{tabular}

\subsection{Summary of Testing Method}

This chapter covers the procedures that we developed to test the accuracy of the moving window technology. We began by introducing the generation of test permeability fields and production data sets, then tested the accuracy of $k h$ as a proxy for the VBY. The results of the VBY validation study demonstrate that VBY serves as a reasonable proxy for $k h$, and that VBY can be estimated reasonably from the $2 \mathrm{D}$ regression of the moving window technology.

To get the same resolution for simulation results and Mosaic results we converted the simulation results from a well basis to a well region basis. After the introduction of two concepts, Max_New_Well_BY and Avg_New_Well_BY, we decided to use the Avg_New_Well_BY as the standard for benchmarking the moving window technology. 
The introduction of DF is to ensure the valid comparisons of infill well performance prediction between cases. The DFs of the four cases verified that all four cases were at a similar stage of depletion and they are comparable. 


\section{CHAPTER IV}

\section{TESTING RESULTS AND RESERVOIR PARAMETER EFFECTS}

In this chapter we will study the effects of different reservoir parameters on the accuracy of moving window technology.

\subsection{Effect of Heterogeneity}

From the parameters of the four cases in Table 3.1, we know that the reservoirs become more heterogeneous as the case number increases. To determine the effect of heterogeneity on the estimation accuracy from moving window technology, we compared the results from Mosaic technology and reservoir simulation.

Figs. 4.1 to 4.4 compare infill BY from the moving window technique to simulation for different degrees of reservoir heterogeneity. The figures grade from the homogeneous (Case 1, Fig. 4.1) to the most heterogeneous (Case 4, Fig. 4.4). It is clear that, as the reservoir becomes more heterogeneous, the data become more scattered and the moving window results become less accurate.

In Case 1 (Fig. 4.1), the moving window technology never underestimates or overestimates by more than $50 \% ; 99 \%$ of the data points fall within the $+/-30 \%$ area. In Case 2 (Fig. 4.2), the moving window technology underestimates 10 wells by more than 50\%. In Cases 3 and 4 (Figs. 4.3 and 4.4), the number of underestimated and overestimated wells increases dramatically; in Case 4, 22 wells are underestimated by $50 \%$ or more and 11 wells are overestimated by $50 \%$ or more. 


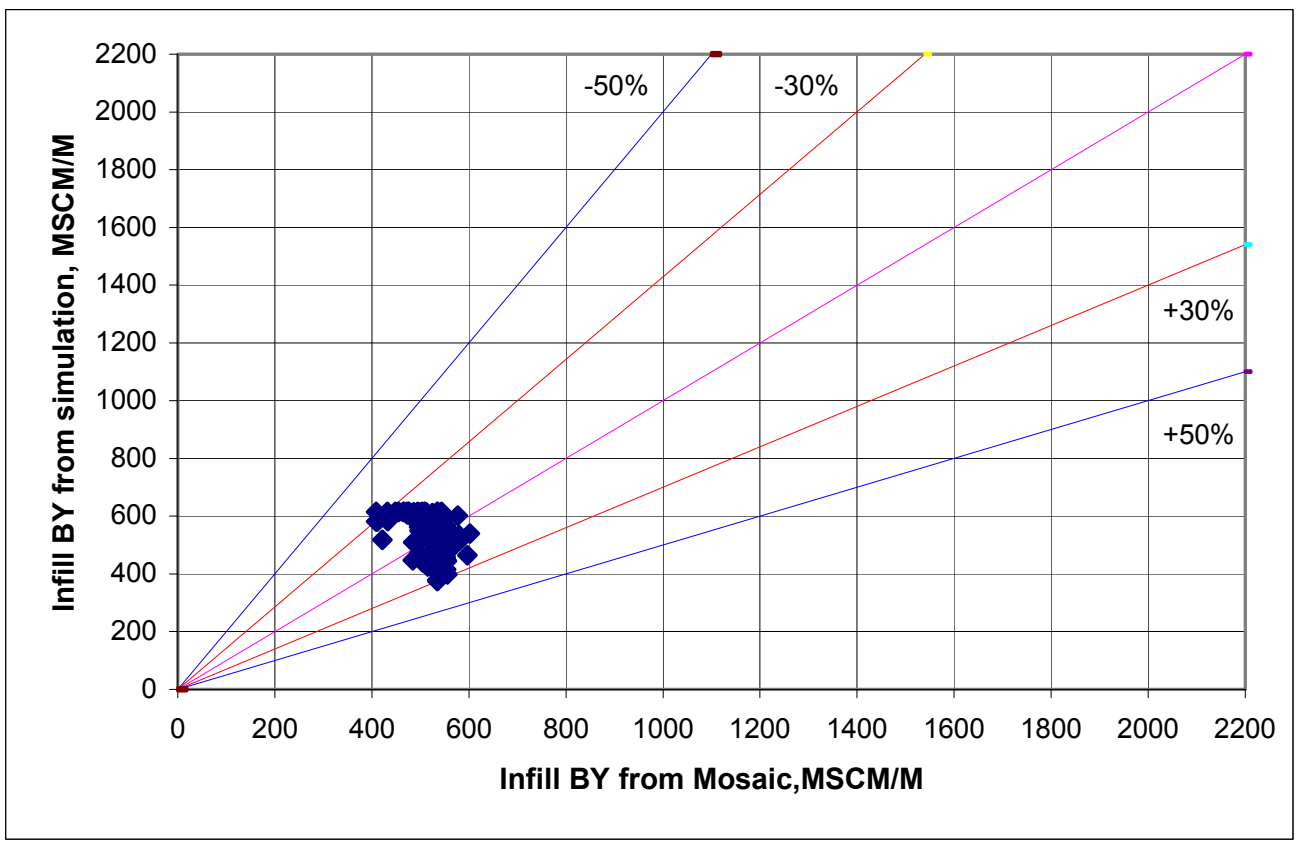

Fig. 4.1 Comparison of infill BY from Mosaic and simulation for Case 1. Infill BY from Mosaic technology shows little deviation from simulation for this case.

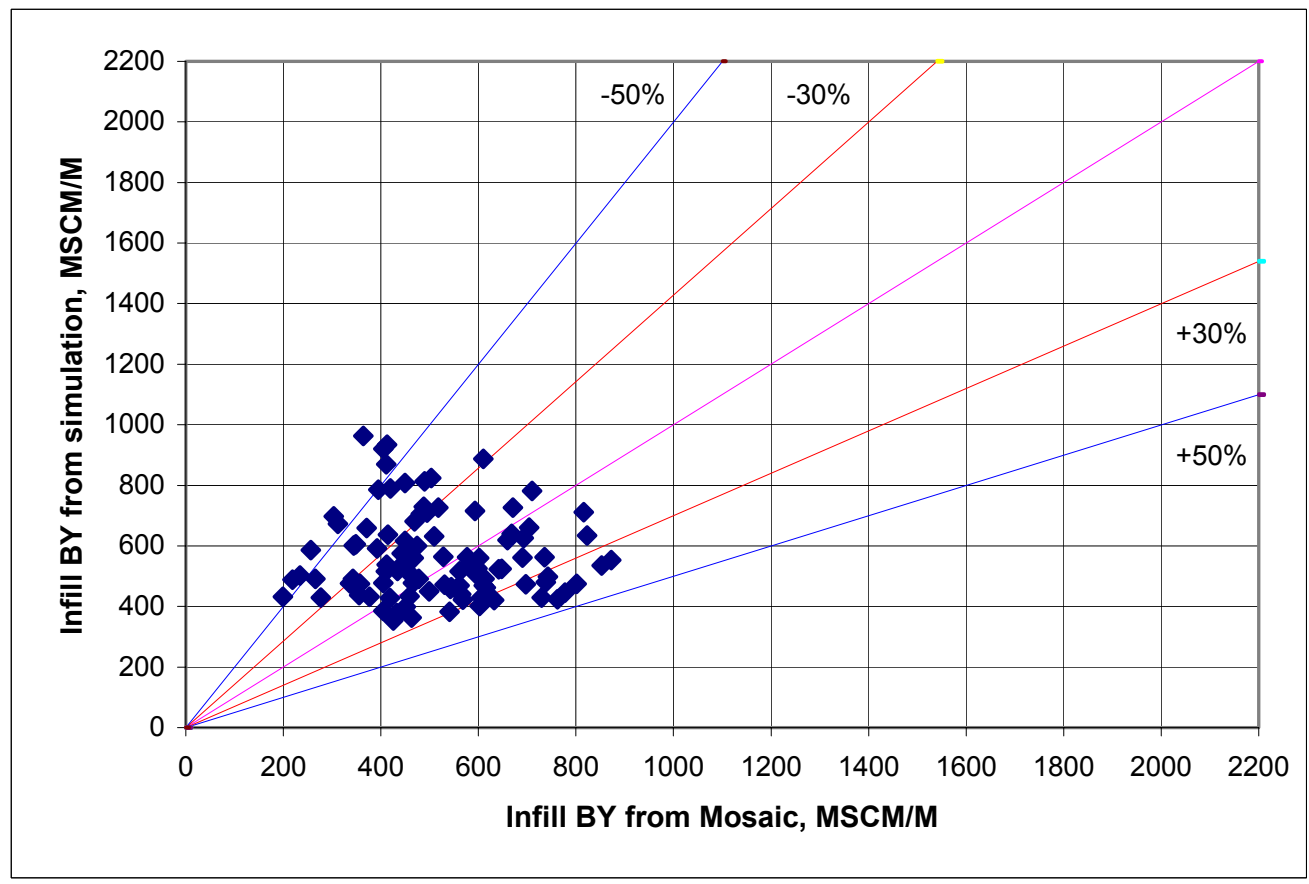

Fig. 4.2 Comparison of infill BY from Mosaic and simulation for Case 2. Infill BY from Mosaic technology correlates well with simulation for this case. 


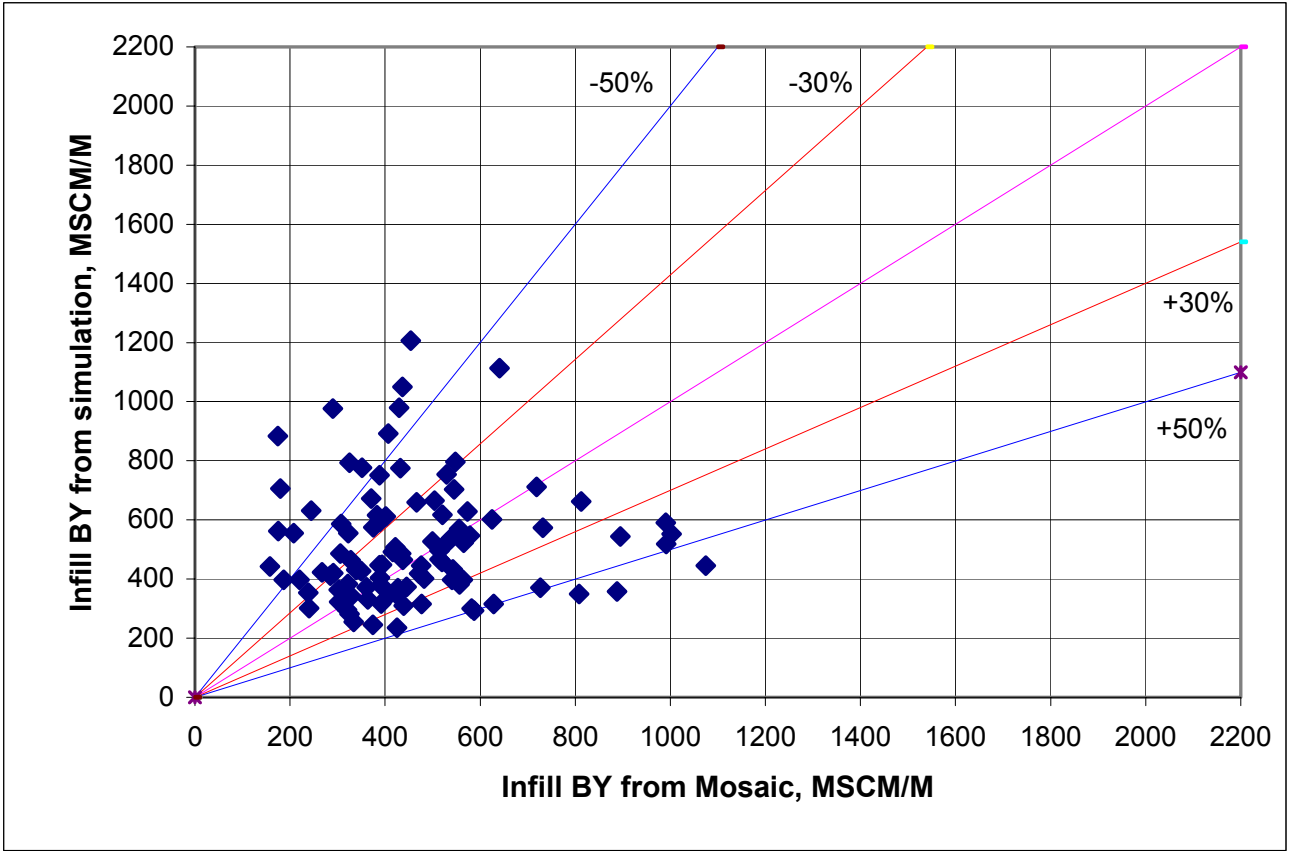

Fig. 4.3 Increased heterogeneity in Case 3 introduces scatter between infill BY from Mosaic and simulation.

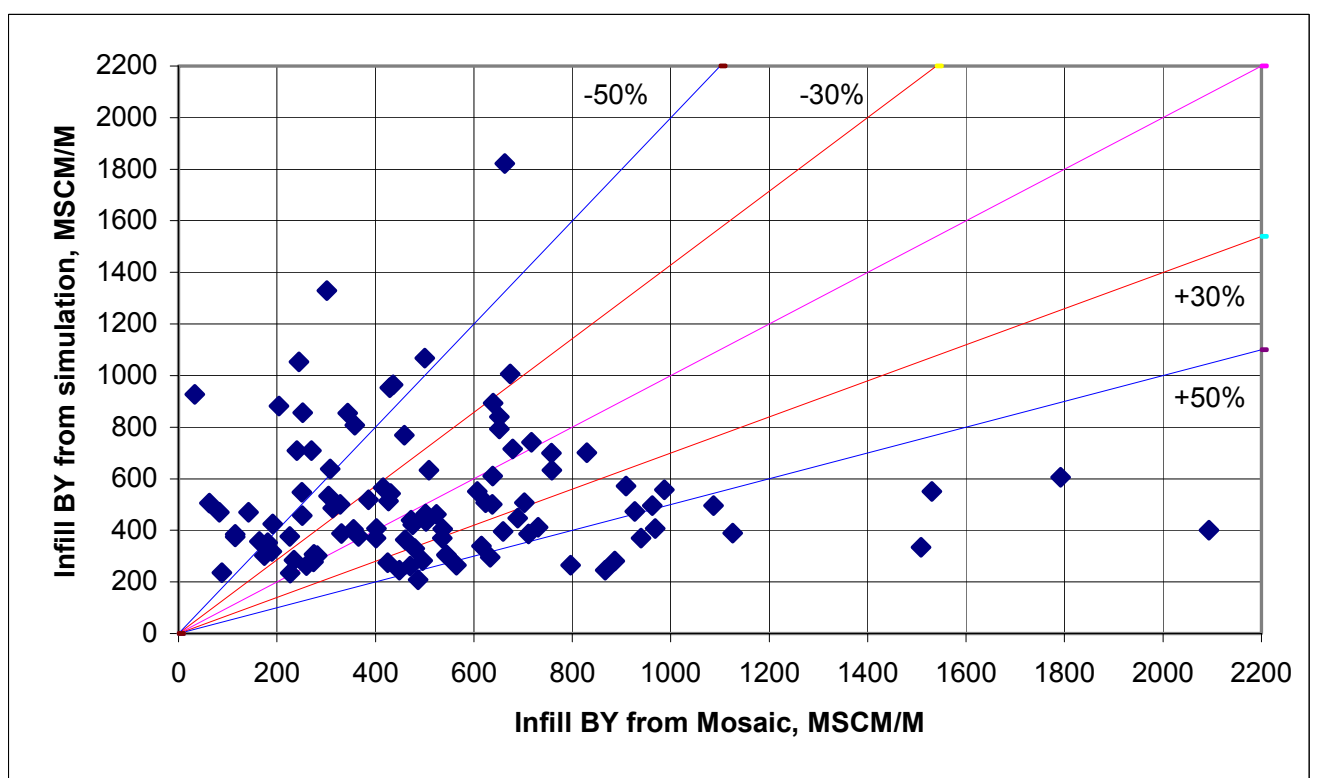

Fig. 4.4 High heterogeneity of Case 4 results in great scatter in infill BY from Mosaic and simulation. 
To help quantify the effect of heterogeneity on the accuracy of moving window results, we use an average percentage difference (APD) as a measure of the deviation from the unit-slope line for each of the four cases. The APD is calculated by the following equation:

$$
\mathrm{APD}=100 * \frac{2}{N}\left(\sum \frac{d_{i}}{r_{i}}\right)=\frac{200}{N} \sum\left(\frac{\left|x_{i}-y_{i}\right|}{x_{i}+y_{i}}\right),
$$

where

$$
d_{i}=\frac{\sqrt{2}}{2} *\left|x_{i}-y_{i}\right|
$$

and

$$
r_{i}=\left(\sqrt{2} * x_{i}-\sqrt{\frac{2}{2}}\left(x_{i}-y_{i}\right)\right)=\frac{\sqrt{2}}{2}\left(x_{i}+y_{i}\right)
$$

Table 4.1 shows the APD for the four cases. The APD increases from $16 \%$ for the homogeneous case to $56 \%$ for the most heterogeneous case, Case 4 . APD increases as the scatter about the unit-slope line increases.

Table 4.1 also shows the averages of infill BYs from the moving window technique and simulation for different degrees of reservoir heterogeneity. Despite instances of large errors in estimation of infill performance for individual wells, the moving window technique predicts the average infill-well performance well. The average relative error ranges from $-11.2 \%$ to $2.3 \%$ for the four cases. The moving window technique appears to provide a conservative estimate of average infill-well performance. For all the cases studied in this work, the estimated average infill performance is either very close to or below the simulated values. 
Table 4.1-Comparison From Mosaic and Simulation Methods

\begin{tabular}{|c|c|c|c|c|}
\hline Case & $\begin{array}{c}\text { Avg percentage } \\
\text { difference, } \\
\%\end{array}$ & $\begin{array}{c}\text { Avg infill BY } \\
\text { from Mosaic, } \\
\text { MSCM/M }\end{array}$ & $\begin{array}{c}\text { Avg infill BY } \\
\text { from simulation, } \\
\text { MSCM/M }\end{array}$ & $\begin{array}{c}\text { Relative error } \\
\text { in infill BY, } \\
\%\end{array}$ \\
\hline 1 & 16 & 513 & 543 & -5.5 \\
\hline 2 & 32 & 517 & 562 & -8.0 \\
\hline 3 & 38 & 459 & 517 & -11.2 \\
\hline 4 & 56 & 533 & 521 & 2.3 \\
\hline
\end{tabular}

\subsection{Causes for Inaccurate Predictions}

In an effort to explain why the moving window technique does not accurately predict the infill-well performance for certain individual wells, we closely examined the wells for which the technique overestimated or underestimated by more than $50 \%$.

The primary reason for inaccurate predictions is unsampled high local variability in permeability. For example, the moving window technique will underestimate the performance of an infill well offsetting a low-permeability well if there is high permeability located nearby that is not sampled by a well. The moving window technique is based on analysis of well locations and production data; thus, if no wells are drilled in local regions of high permeability, the moving window technique will not be able to predict higher infill performance for the particular area. The opposite situation also occurs, resulting in overestimates of infill performance in certain cases.

Some of the larger differences occur in sparsely drilled regions of the reservoir. When the number of wells in a particular window is inadequate, the moving window technique defaults to a regional or global correlation, instead of a local correlation. A 
regional or global correlation obviously will not predict local performance as accurately as a local correlation.

\subsection{Effect of Average Permeability}

The average permeability of the cases presented thus far is $0.2 \mathrm{md}$. To determine the effect of permeability level on the estimation results, we also ran another homogeneous reservoir case. We increased the average permeability to $1.0 \mathrm{md}$ and compared the results with the 0.2 md reservoir case.

Figs. 4.1 and 4.5 show the comparisons of infill well performance predicted by the moving window technique to that predicted by simulation for homogeneous permeability distributions of 0.20 and $1.0 \mathrm{md}$, respectively. For the $0.2 \mathrm{md}$ case (Fig. 4.1), most of the points are within $30 \%$ of the values from simulation. The average infill $\mathrm{BY}$ from moving window technology is $513 \mathrm{MSCM} / \mathrm{M}$ and the average infill $\mathrm{BY}$ from simulation is $543 \mathrm{MSCM} / \mathrm{M}$. Thus, on average, the moving window technique underestimates the infill BY by about $6 \%$. This small error is noteworthy, considering that the reservoir has experienced depletion resulting in a $12 \%$ decrease in well productivity. 


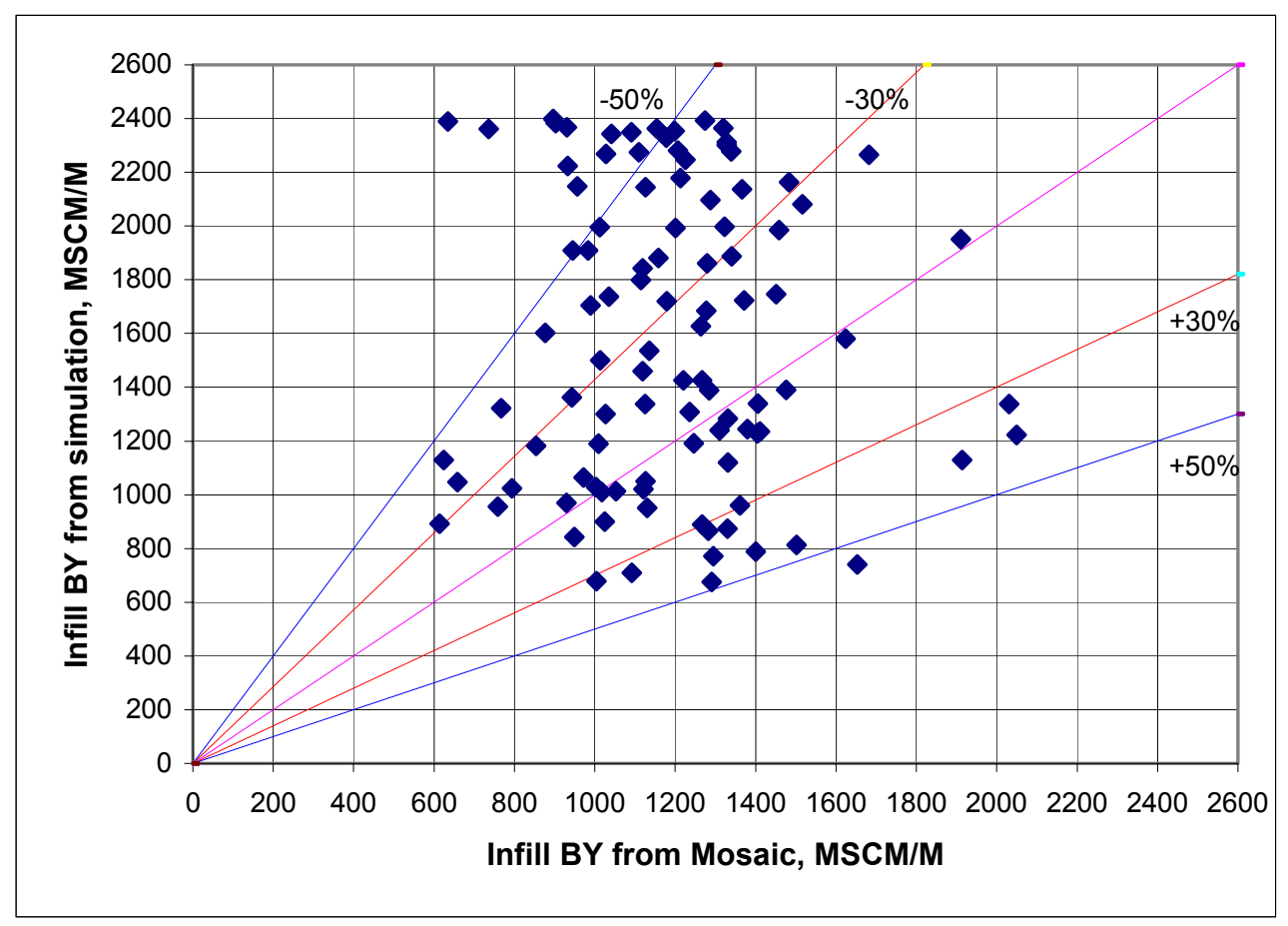

Fig. 4.5 Infill BY from Mosaic shows low correlation with simulation for $1.0 \mathrm{md}$ homogeneous reservoir.

The error increases as the permeability increases; Fig. $\mathbf{4 . 5}$ shows the comparison for the $1.0 \mathrm{md}$ reservoir. There is much more scatter in the points; several points differ by more than $50 \%$ from the simulated values. The moving window technique underestimates the average infill BY by $25 \%$ for this case, as compared to $6 \%$ for the 0.2 md case. The larger error is attributed to greater depletion, $40 \%$ for the $1.0 \mathrm{md}$ case, which results when permeability is increased and everything else stays the same.

\subsection{Effect of Search Area}

The default value for the local search area in the moving window technology is 3,000 acres. To find the effect of the search area, we varied the search areas from 1,000 to 12,000 acres in the analysis of Case 3. Results are presented in Table 4.2 and Fig. 4.6. 
Table 4.2-Effect of Search Area on Relative Error for Case 3

\begin{tabular}{|c|c|c|c|}
\hline $\begin{array}{c}\text { Search area, } \\
\text { acre }\end{array}$ & $\begin{array}{c}\text { Infill BY from } \\
\text { Mosaic, } \\
\text { MSCM/M }\end{array}$ & $\begin{array}{c}\text { Infill BY from } \\
\text { simulation, } \\
\text { MSCM/M }\end{array}$ & $\begin{array}{c}\text { Relative error } \\
\text { of infill BY, } \\
\%\end{array}$ \\
\hline 1,000 & 480.8 & 517.2 & -7.1 \\
\hline 1,500 & 458.6 & 517.2 & -11.2 \\
\hline 2,000 & 498.9 & 517.2 & -3.6 \\
\hline 3,000 & 458.5 & 517.2 & -11.2 \\
\hline 6,000 & 424.1 & 517.2 & -18.0 \\
\hline 12,000 & 409.6 & 517.2 & -20.7 \\
\hline
\end{tabular}

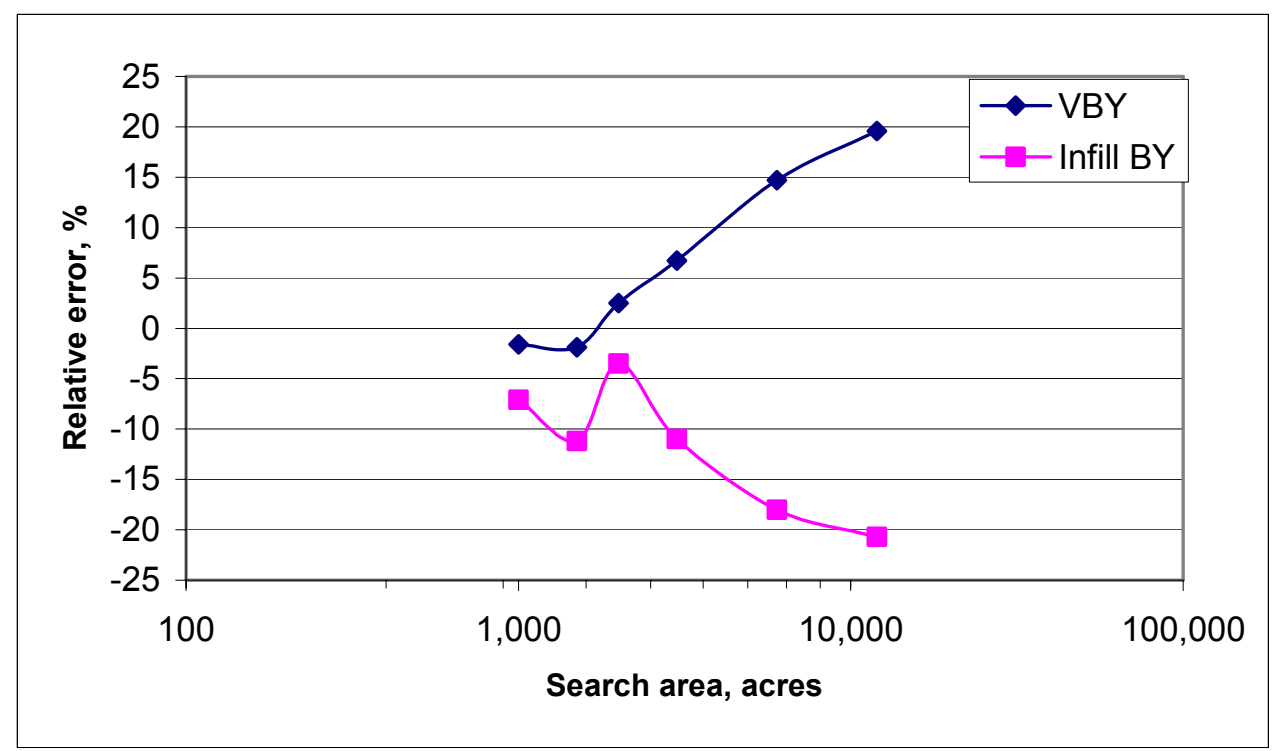

Fig. 4.6 Relative error of VBY and infill BY vs. search area for Case 3.

From Table 4.2 we see the smaller domain size results are better for Case 3. As we know from Table 4.1, the reservoir of Case 3 is very heterogeneous. So there is a larger variation in reservoir properties in bigger domains than in smaller domains. These 
results indicate that if the domain size is greater than about 3,000 acres, the relative error of the infill BY begins to increase significantly.

Fig. 4.6 indicates that the moving window technology will overestimate VBY with increasing of search area size. The overestimation of VBY is likely a large part of the cause for underestimation of infill BY with increasing search area size. Note the non-monotonic behavior of the relative error of infill BY. We are not sure what causes that behavior. But we do know that the search area of 2,000 acres (corresponding to the smallest relative error of infill BY in Table 4.2) is very close to the correlation length of the permeability distribution for Case 3 (1,825 acres).

From the analysis presented above, we know that the size of the search area will have a significant effect on the estimation accuracy of the moving window technology. The search-area size will be the first question we need to answer when we apply this technology to a new area. Optimal search area sizes will be small enough to avoid large changes in regional reservoir properties, e.g., permeability, and large enough to have at least 3 to 5 wells per window.

\subsection{Effect of Well Spacing}

The moving window technology will calculate the well's initial well spacing and current well spacing in the data processing. The current well spacing is used as a proxy for the drainage area. We want to determine the effect of well spacing on the estimation results of the moving window technology. But the well spacings of the 100 wells in the four cases that we ran are not the same. It is very difficult to draw any conclusions with regard to well spacing from these 100 -well cases.

To investigate the effects of nonuniform well spacing, we ran additional cases with the same permeability distribution and a uniform arrangement of wells. These cases are named Case 1a, Case 2a, Case 3a and Case 4a. The reservoir properties of these 
cases are shown in the Table 3.1. The difference between Case 1 and Case 1a is the number of wells in the study area. The date for the infill wells in Cases 1a to 4a is 1984, two years after the latest infill-drilling campaign.

In Cases 1a to 4a, 169 wells were drilled at a uniform well spacing of 1,280 acres in 1962. Fig. 4.7 shows the initial well spacing of the 169 wells. The first round of 169 infill wells was drilled on 640-acre spacing in 1972. The second round of 338 infill wells was drilled on 320 acres in 1982. Figs. 4.8 and 4.9 show the well spacings after the two infill-drilling campaigns. There were 676 wells in the study area after the second infill drilling campaign. The analysis was performed as of 1984, two years after the last round of infill wells. 


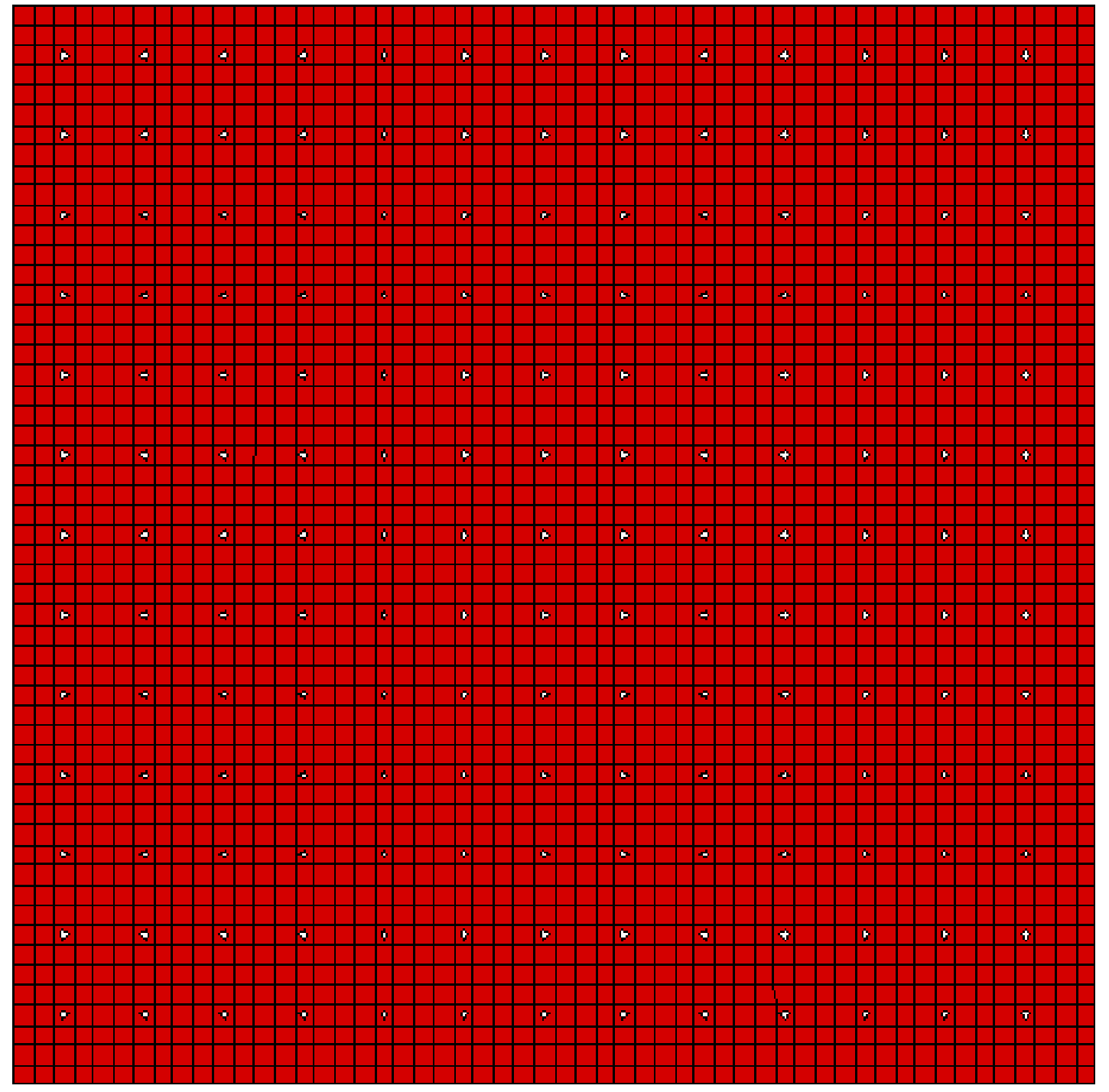

Fig. 4.7 The initial well spacing of 169 wells in 1962. 


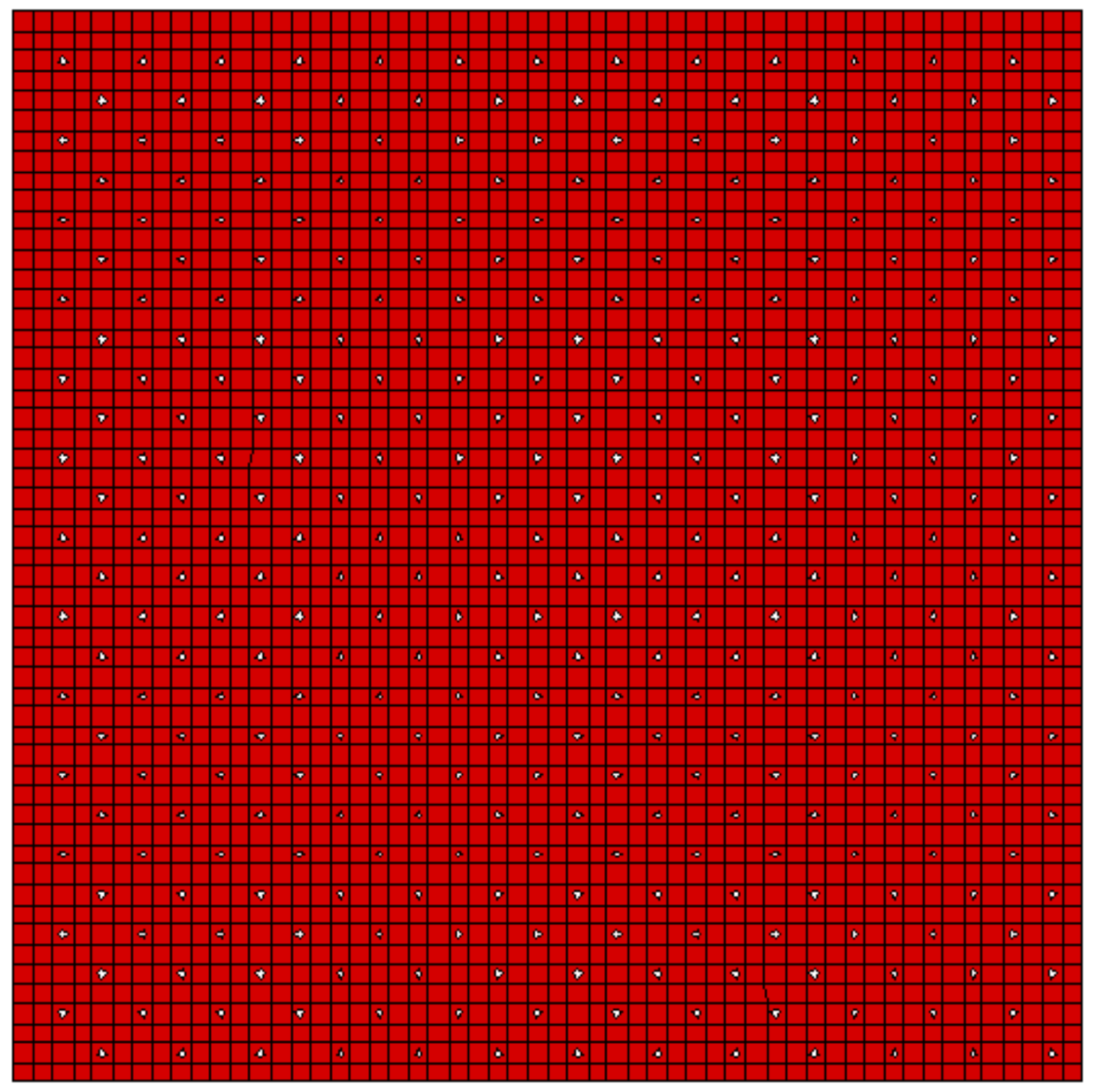

Fig. 4.8 The well spacing after the first round of infill-drilling in 1972. 


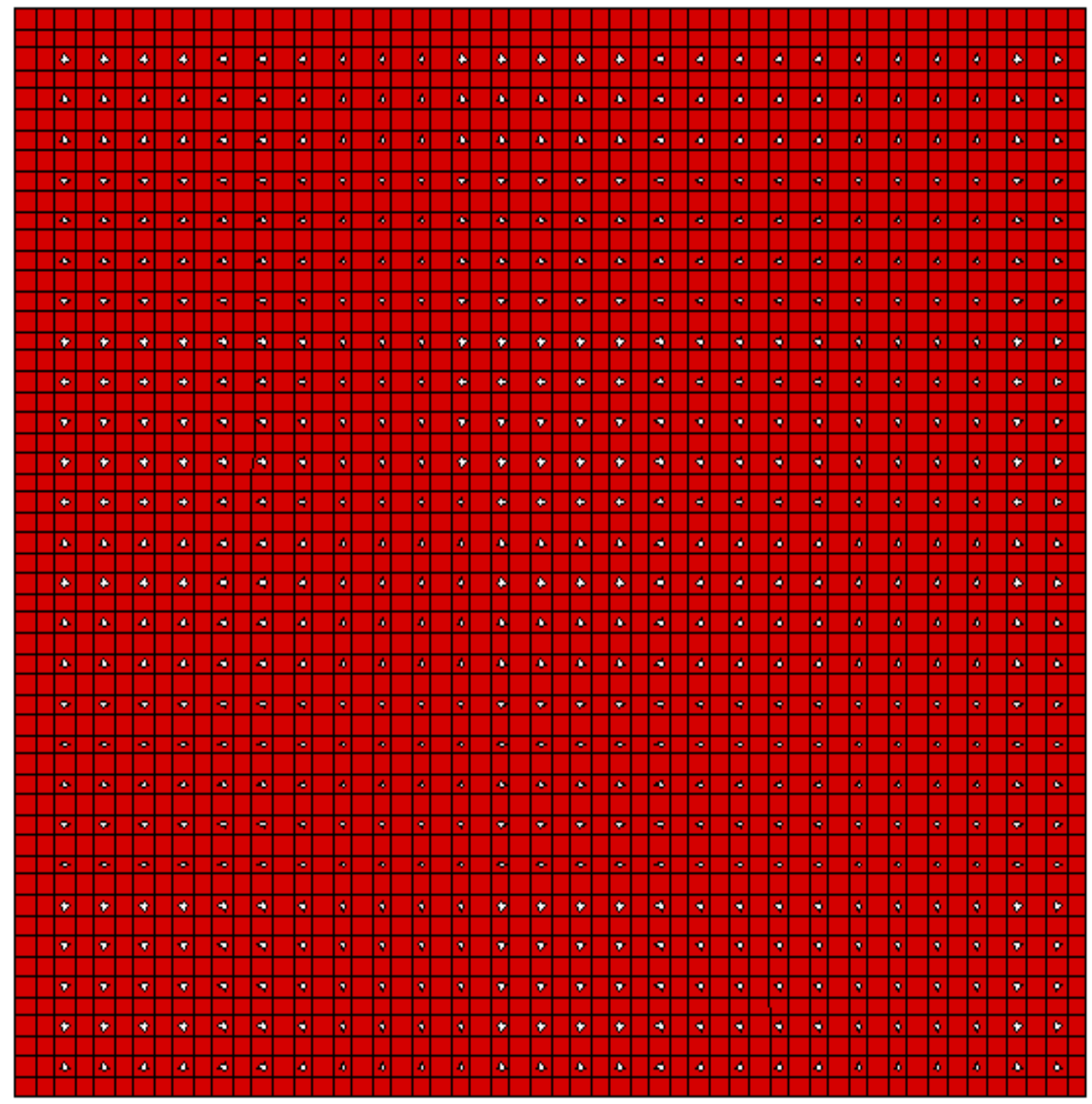

Fig. 4.9 The well spacing after the second round of infill-drilling in 1982.

Table 4.3 shows the results of the four cases from simulation and moving window technology. The two results are very close, except Case 4a, when we consider the wells as a group. The relative error of infill BY for Cases 1a to $3 \mathrm{a}$ varies from $-3.3 \%$ to $-1.6 \%$. But there is a big difference in the most heterogeneous case, Case $4 \mathrm{a}$. When we decreased the search area from 3,000 acres to 1,500 acres (which we call Case $4 \mathrm{a}^{*}$ ), 
the relative error of infill BY dropped from $27.7 \%$ to $2.3 \%$. Apparently, the search area of 3,000 acres is too large for Case 4a.

While there is no big difference among the four cases except for Case $4 \mathrm{a}$, on average the relative error of infill BY becomes more scattered as the case number and heterogeneity increase. In Case 1a, $100 \%$ of the data points fall within the $+/-30 \%$ area, but only about $30 \%$ of the data points fall within $+/-30 \%$ in Cases $4 \mathrm{a}$ and $4 \mathrm{a}^{*}$.

The average percentage differences (APDs) calculated from the homogenous case, Case $1 \mathrm{a}$, and the most heterogeneous case, Case $4 \mathrm{a}$ and $4 \mathrm{a}^{*}$, are larger than those calculated from Case 1 and Case 4 (Table 4.1). But APDs from Case 2a and Case 3a are smaller than those from Case 2 and Case 3.

Table 4.3-Results From Uniform Spacing With Boundary Wells

\begin{tabular}{|c|c|c|c|c|c|c|}
\hline Case & $\begin{array}{c}\text { Avg } \\
\text { percentage } \\
\text { difference, } \\
\%\end{array}$ & $\begin{array}{l}\text { Avg infill } \\
\text { BY from } \\
\text { simulation, } \\
\text { MSCM/M }\end{array}$ & $\begin{array}{l}\text { Avg infill } \\
\text { BY from } \\
\text { Mosaic, } \\
\text { MSCM/M }\end{array}$ & $\begin{array}{c}\text { Avg relative } \\
\text { error of } \\
\text { infill BY, } \\
\quad \%\end{array}$ & $\begin{array}{c}\% \text { points in } \\
-30 \% \text { to } \\
+30 \% \text { area, } \\
\%\end{array}$ & $\begin{array}{c}\% \text { points in } \\
<-50 \% \text { and } \\
>+50 \% \text { area, } \\
\%\end{array}$ \\
\hline $1 \mathrm{a}$ & 6 & 389 & 377 & -3.1 & 100 & 0 \\
\hline $2 a$ & 20 & 387 & 381 & -1.6 & 85.2 & 0 \\
\hline $3 a$ & 36 & 365 & 353 & -3.3 & 56.1 & 15.3 \\
\hline $4 a$ & 51 & 341 & 267 & -27.7 & 38.9 & 32.0 \\
\hline $4 a^{*}$ & 68 & 341 & 349 & +2.3 & 29.6 & 48.2 \\
\hline
\end{tabular}

Notes:

- The search areas for Case 1a to 4a are 3,000 acres.

- The search area for Case $4 \mathrm{a}^{*}$ is 1,500 acres.

The reservoir we used in the reservoir simulator is a closed-boundary reservoir, so the result of the moving window technology will be influenced by the reservoir 
boundaries. To find the reservoir boundary's effect on the moving window technology, we take only the inner 576 wells data into consideration (ignoring the 100 boundary wells in our study reservoir).

Table 4.4 shows the results of the 576 inner wells from reservoir simulation and moving window technology. The accuracy of average infill BY estimation by moving window technology improves about $1 \%$, the average percent difference improves as wells.

Table 4.4-Results From Uniform Well Spacing Without Boundary Wells

\begin{tabular}{|c|c|c|c|c|c|c|}
\hline Case & $\begin{array}{c}\text { Avg } \\
\text { percentage } \\
\text { difference, } \\
\%\end{array}$ & $\begin{array}{l}\text { Avg infill } \\
\text { BY from } \\
\text { simulation, } \\
\text { MSCM/M }\end{array}$ & $\begin{array}{l}\text { Avg infill } \\
\text { BY from } \\
\text { Mosaic, } \\
\text { MSCM/M }\end{array}$ & $\begin{array}{c}\text { Avg relative } \\
\text { error of } \\
\text { infill BY, } \\
\%\end{array}$ & $\begin{array}{c}\% \text { points in } \\
-30 \% \text { to } \\
+30 \% \text { area, } \\
\%\end{array}$ & $\begin{array}{c}\% \text { points in } \\
<-50 \% \text { and } \\
>+50 \% \text { area, } \\
\%\end{array}$ \\
\hline $1 \mathrm{a}$ & 4 & 379 & 370 & -2.3 & 100 & 0 \\
\hline $2 a$ & 16 & 380 & 377 & -0.8 & 85.6 & 0 \\
\hline $3 a$ & 30 & 358 & 352 & -1.7 & 57.1 & 14.8 \\
\hline $4 a$ & 50 & 335 & 253 & -24.4 & 39.2 & 30.7 \\
\hline $4 a^{*}$ & 60 & 335 & 338 & +0.8 & 26.0 & 59.9 \\
\hline
\end{tabular}

Notes:

- The search areas for Case 1a to 4a are 3,000 acres.

- The search area for Case $4 \mathrm{a}^{*}$ is 1,500 acres.

On the basis of the two sets of results, Table 4.3 and Table 4.4, we see that the reservoir boundary does not have much effect on the infill BY estimation from the moving window technology.

The results from moving window technology for Cases 1a to 3a compare more closely to simulation results than for the real-world 100-well cases discussed in Cases 1 
to 3 . Both the average of individual well percent differences and the percent error in average infill BY are significantly lower for the uniformly-spaced Cases $1 \mathrm{a}$ to $3 \mathrm{a}$ than the nonuniformly spaced Cases 1 to 3 (Table 4.1 and Table 4.4). The depletion in Cases 1a to $3 \mathrm{a}$ is more uniform than the depletion in Case 1 to 3 because the wells are evenly spaced in Cases 1a to 3a. The more uniform depletion may cause the relative error of average infill BY to decrease.

The results from Case 4 and Case $4 \mathrm{a}$, the most heterogeneous cases, are somewhat anomalous. The error in average infill BY for the uniformly spaced Case $4 \mathrm{a}$ is substantially larger than the less heterogeneous Cases 1a-3a and it is also much larger than the error of average infill BY of Case 4 (Table 4.1 and Table 4.4).

We speculate that this anomalous behavior may result from the relationship between the permeability correlation length and the window size used in the moving window analysis; i.e., when the window size is large relative to the permeability correlation length, the moving window analysis becomes less reliable. To investigate the result from Case 4a, we reran the moving window analysis with a smaller window size, 1,500 acre, in Case $4 \mathrm{a}^{*}$. The results are shown in Tables 4.3 and 4.4. With a smaller search area in Case $4 \mathrm{a}^{*}$, its results are comparable with other cases and the error in average infill BY decreased significantly to less than 3.0\%. The APD also decreases from $68 \%$ to $60 \%$ for Case $4 a^{*}$, and the percentage of data points in the $+/-30 \%$ area decrease from $29.6 \%$ to $26 \%$.

Fig. 4.10 shows the results from several runs for Case $4 a$ with different search areas. This figure indicates that the error increases significantly when the ratio of window size to permeability correlation area exceeds a certain threshold value. The line in Fig. 4.10 is a curve-fit and it does not reflect behavior predicted from an analytical model. 


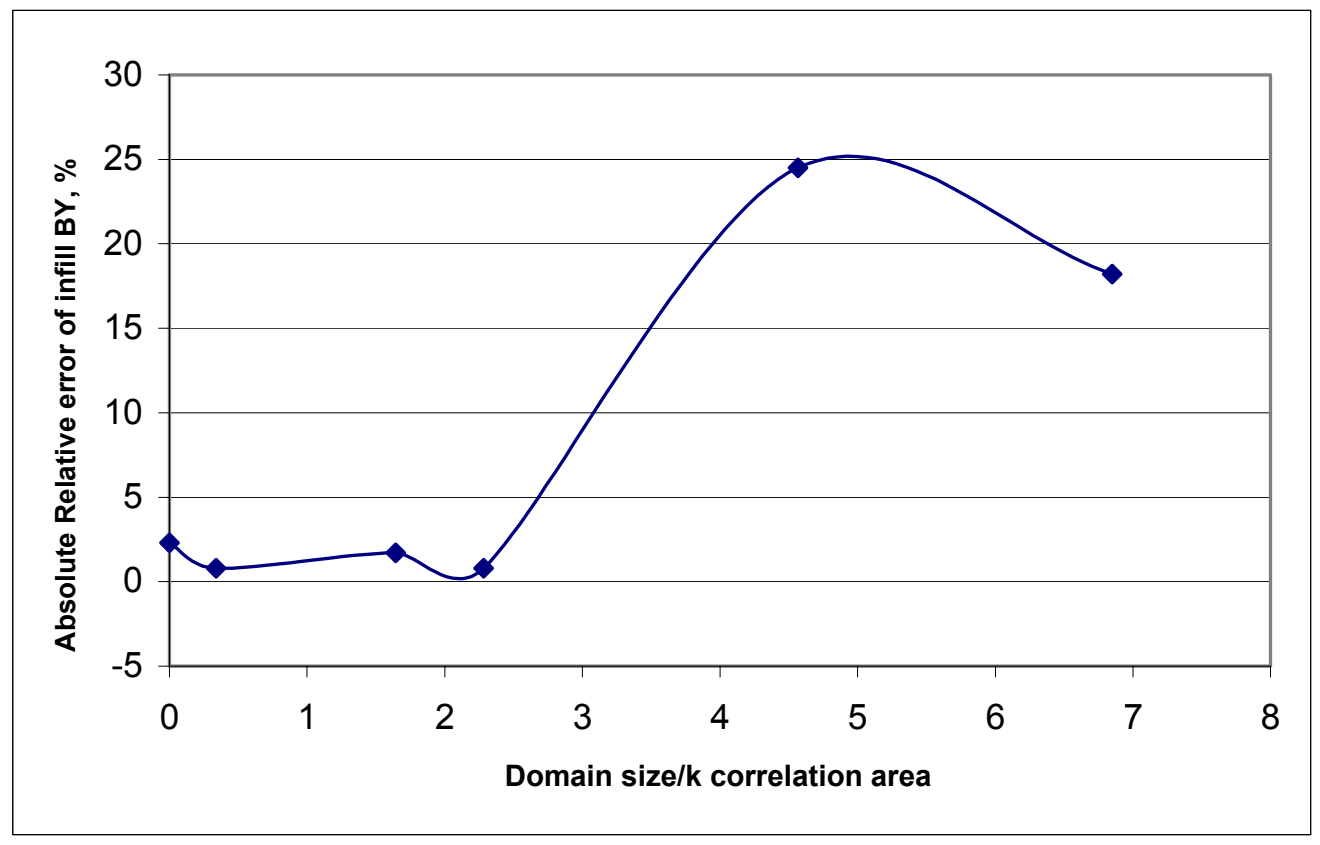

Fig. 4.10 Absolute relative error with the domain size.

We did not study the effect of domain size on other cases. The threshold value of domain size might vary for reservoirs with different degrees of heterogeneity.

We generally do not know the reservoir permeability distribution for actual fields. So determining the optimum domain size from production data will be a demanding task in the application of the moving window technology. In Section 4.7 we discuss how we can get some information about the permeability distribution from production data.

\subsection{Effect of the Date for the Infill Wells on the Analysis}

The objective of this part of the study was to determine if the time difference between the date for the infill wells and the latest wells' date of first production in our study area influences the estimation results.

Case 1b (Table 4.5) is identical to Case 1a except that the date for the infill wells is 1991, or 9 years after the last round of infill wells, rather than 2 years after for Case 1 a. 
In Case $1 \mathrm{~b}$ the moving window technology overestimates the infill BY by $20.7 \%$. The average relative error of infill BY increased significantly in Case 1b, suggesting that the estimation error is also related to the timing difference between the dates for the infill wells and the time corresponding to the last round of infill wells.

Table 4.5-Effect of Date for Infill Wells on Moving Window Technology

\begin{tabular}{|c|c|c|c|c|}
\hline Case & $\begin{array}{c}\text { Date for the } \\
\text { infill wells, } \\
\text { year }\end{array}$ & $\begin{array}{c}\text { Infill BY from } \\
\text { simulation, } \\
\text { MSCM/M }\end{array}$ & $\begin{array}{c}\text { Infill BY } \\
\text { from Mosaic, } \\
\text { MSCM/M }\end{array}$ & $\begin{array}{c}\text { Avg relative error } \\
\text { of infill BY, } \\
\%\end{array}$ \\
\hline la & 1984 & 389 & 377 & $-3.1 \%$ \\
\hline lb & 1991 & 284 & 343 & $+20.7 \%$ \\
\hline
\end{tabular}

Fig. 4.11 shows the 2D regression for a typical domain in Case 1a. The solid line is the best-fit line that the moving window technology used to calculate infill VBY. But after the second round of infill drilling of 338 wells, the depletion rate of the closed-boundary reservoir increased from that of the period after the first round of infill drilling. The reservoir depletion rate follows the dashed line after the second round of infilling drilling; it will not follow the best-fit line any more. The ongoing infill drilling campaigns will deplete the reservoir more rapidly than before because of the large number of infill wells. The performance of the latest wells will give us more valuable information on the current reservoir than the old wells.

The difference between the two lines in Fig. 4.11 is not very large at first but increases with time. This suggests that the most accurate estimation time for the infill wells may be soon after the latest round of infill drilling. 


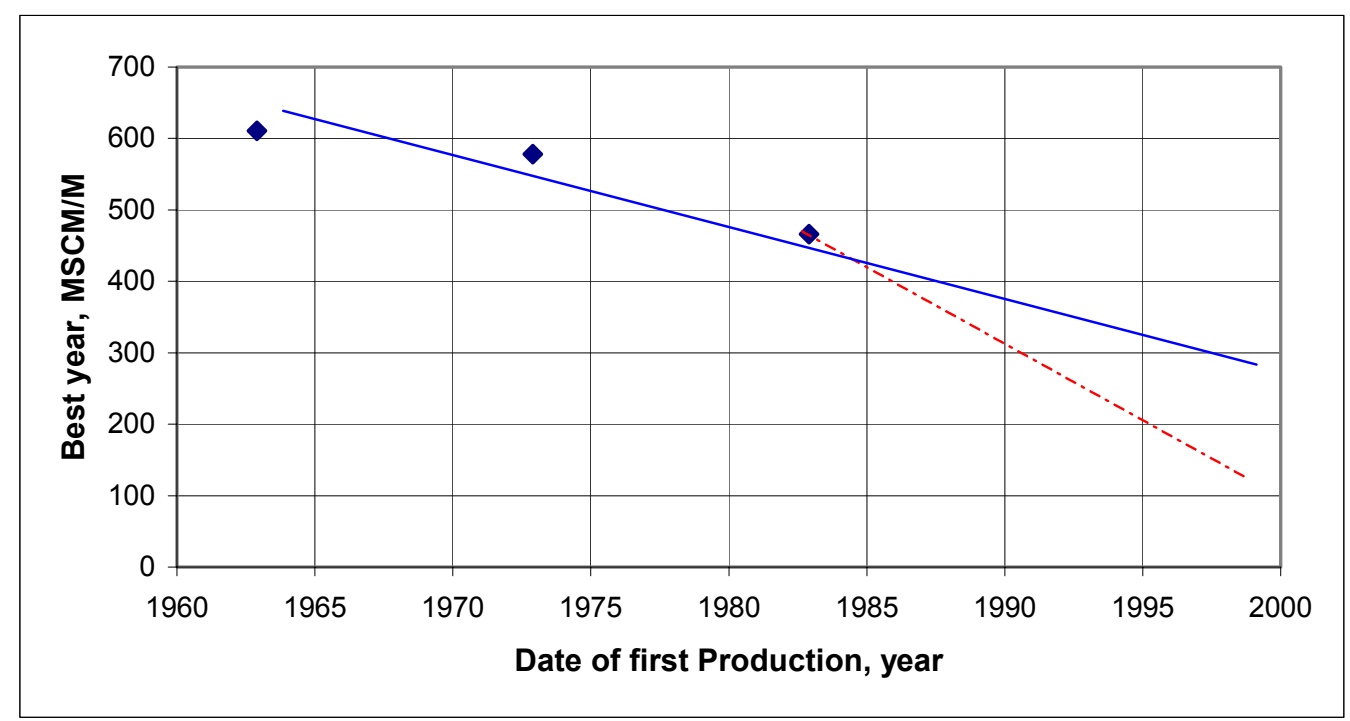

Fig. 4.11 Effect of the timing of the infill wells on the moving window technology (data from Case 1a).

\subsection{Estimation of Reservoir Heterogeneity}

Our results show that the accuracy of infill performance predictions by the moving window technique decreases as heterogeneity increases. Thus, in applying the moving window technique to field data, it is desirable to know the degree of heterogeneity in reservoir properties, particularly permeability.

The data required to quantify the heterogeneity of permeability are usually not available. However, it may be possible to estimate heterogeneity from production data, assuming similarity in well-completion efficiencies.

Fig. 4.12 shows the coefficient of variation, $C_{v}$, of both VBY and BY as a function of the $C_{v}$ of permeability for the four cases (Cases 1 to 4$)$. The $C_{v}$ of VBY and BY calculated by the moving window technology increase as $C_{v}$ of permeability increases. This relationship indicates that it may be possible to estimate heterogeneity from production data, or to augment the estimations of heterogeneity from log and core data. 


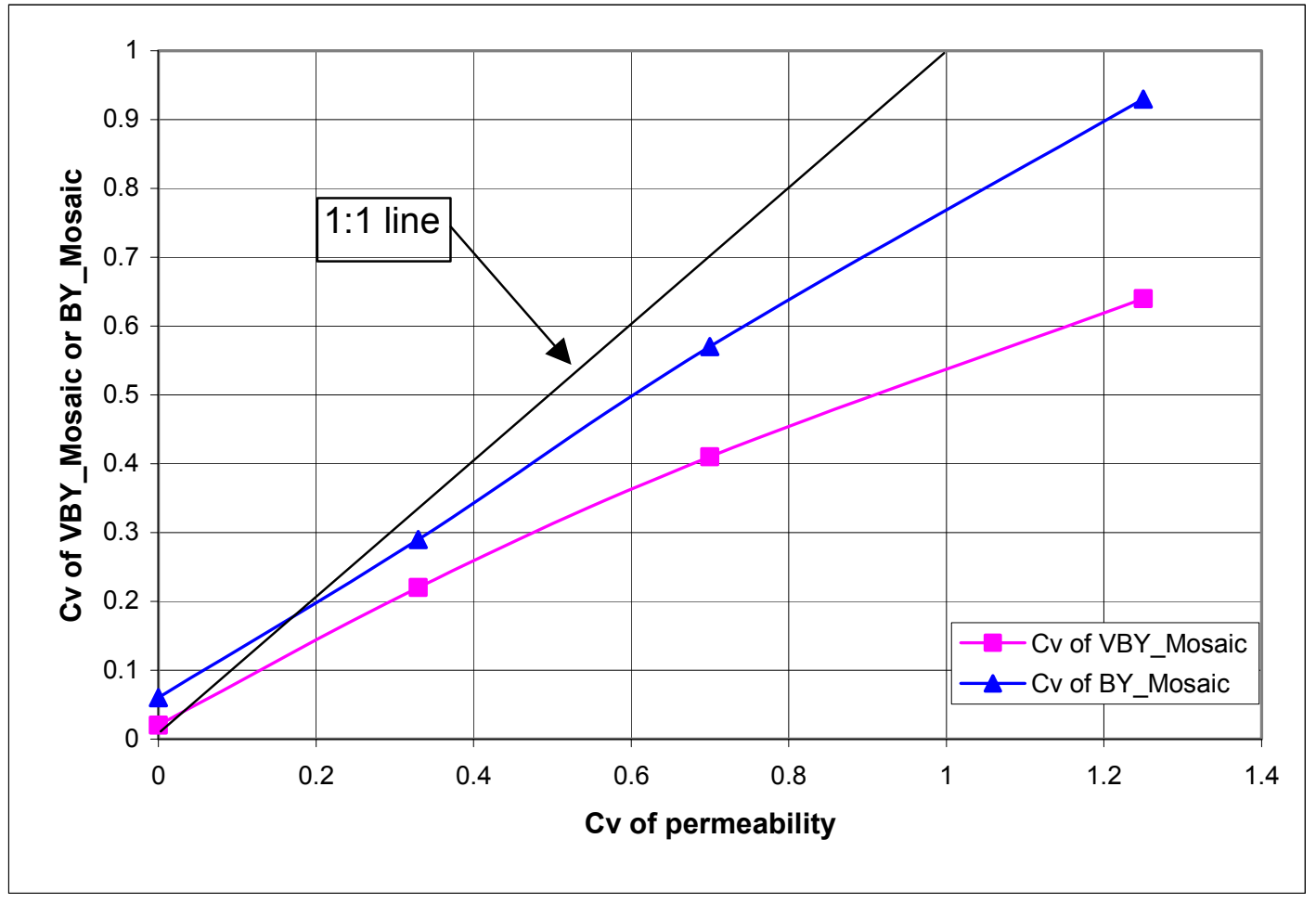

Fig. 4.12 Variability in production data related to variability in permeability.

\subsection{Effect of Number of Wells}

From the analyses so far we know that the moving window predictions for individual wells can be quite far off. Therefore, ranking infill candidates on predicted individual well performance may not necessarily yield the best overall results. Poor wells, predicted to be good wells, may still be drilled and good wells, predicted to be poor wells, may not be drilled at all.

But it appears that moving window technology does well in predicting the average infill well performance for a group of wells. So we should examine the infill-drilling program as for groups of wells when we use this technology to evaluate infill-drilling potential. 
When we use this technology, we can divide a basin or field into smaller areas and predict the distributions of infill performance as a group for the smaller areas, rather than individual wells. Here an important question is how many wells are required to get a reasonably accurate prediction of the average infill-well performance.

To estimate the variability of the predicted average as a function of the number of wells in the group, we randomly selected 50 subsets of 10 wells and, for each subset, determined the absolute difference and the percent difference between the averages of the moving window and simulation infill BY. We then repeated this for 50 subsets of 15 , $20,25, \ldots, 95$ wells.

The results of this analysis for the four unevenly spaced 100-well permeability distributions are shown in Figs. 4.13 to 4.16, which are plots of percent difference in the averages of the moving window and simulation infill BY as a function of $n$, the number of wells in the average. These plots demonstrate that the variability of the predicted average infill-well performance decreases with the number of wells in the average, and shows how many wells are required to obtain a certain variability in the average infill-performance prediction. 


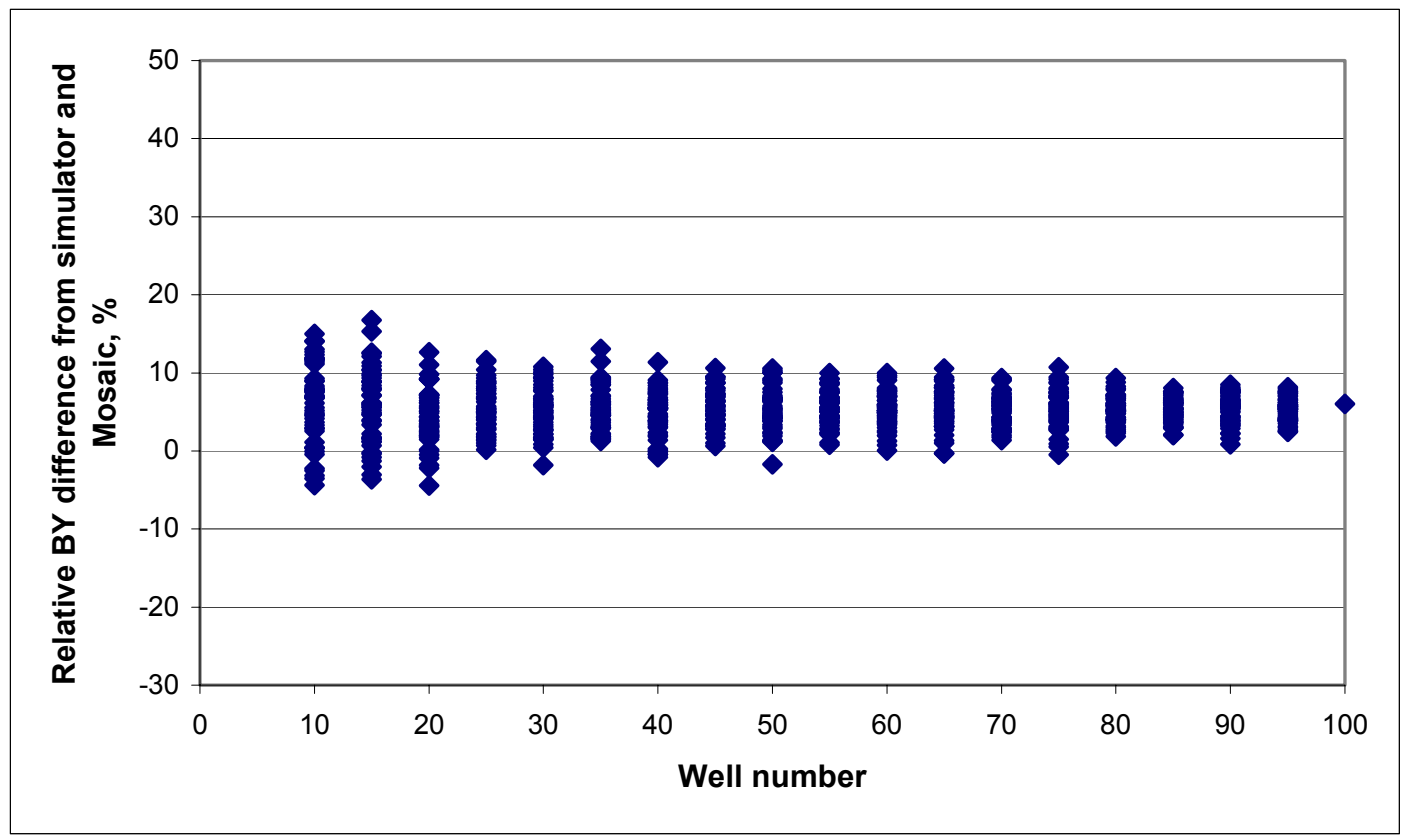

Fig. 4.13 Analysis of variability in error in average infill BY for Case 1.

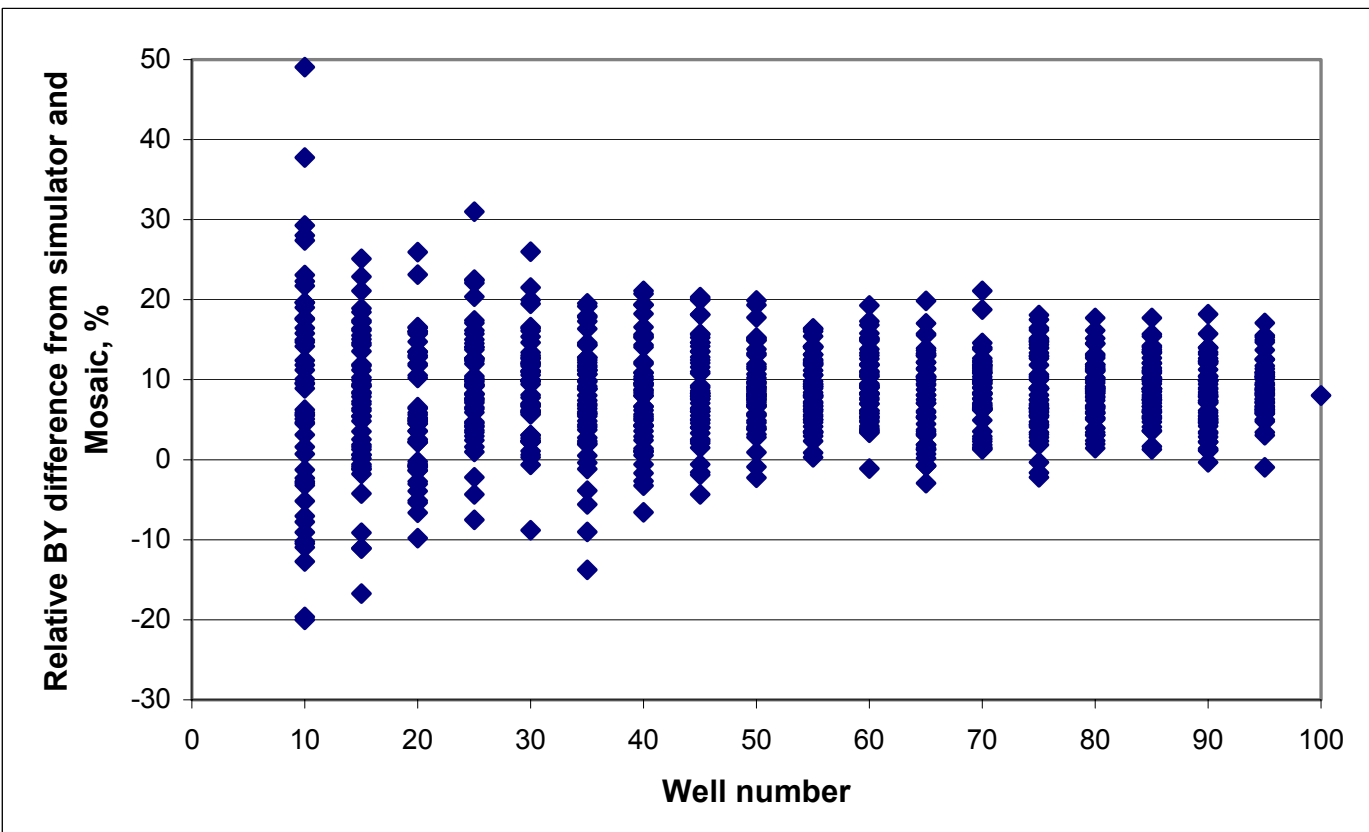

Fig. 4.14 Analysis of variability in error in average infill BY for Case 2. 


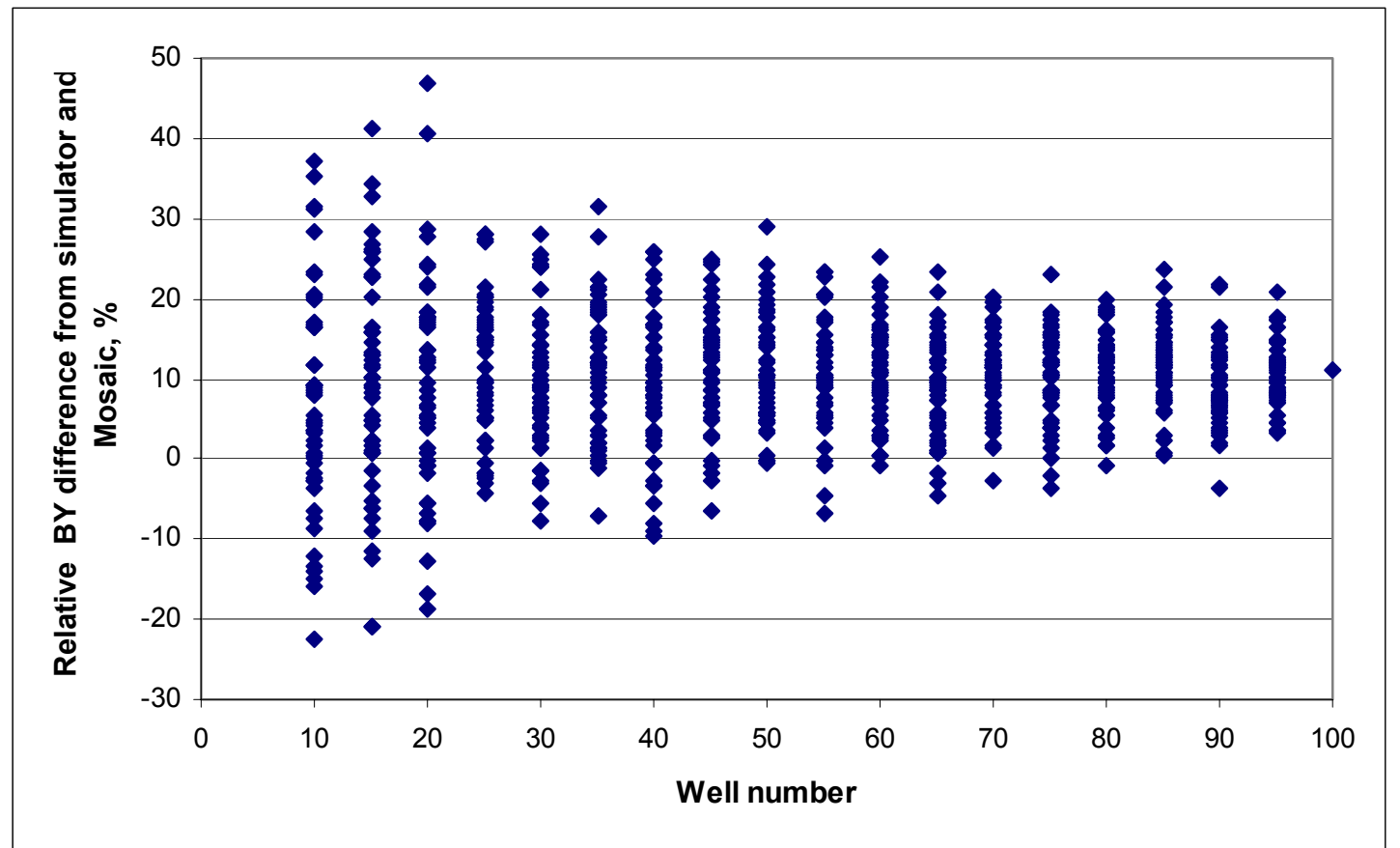

Fig. 4.15 Analysis of variability in error in average infill BY for Case 3.

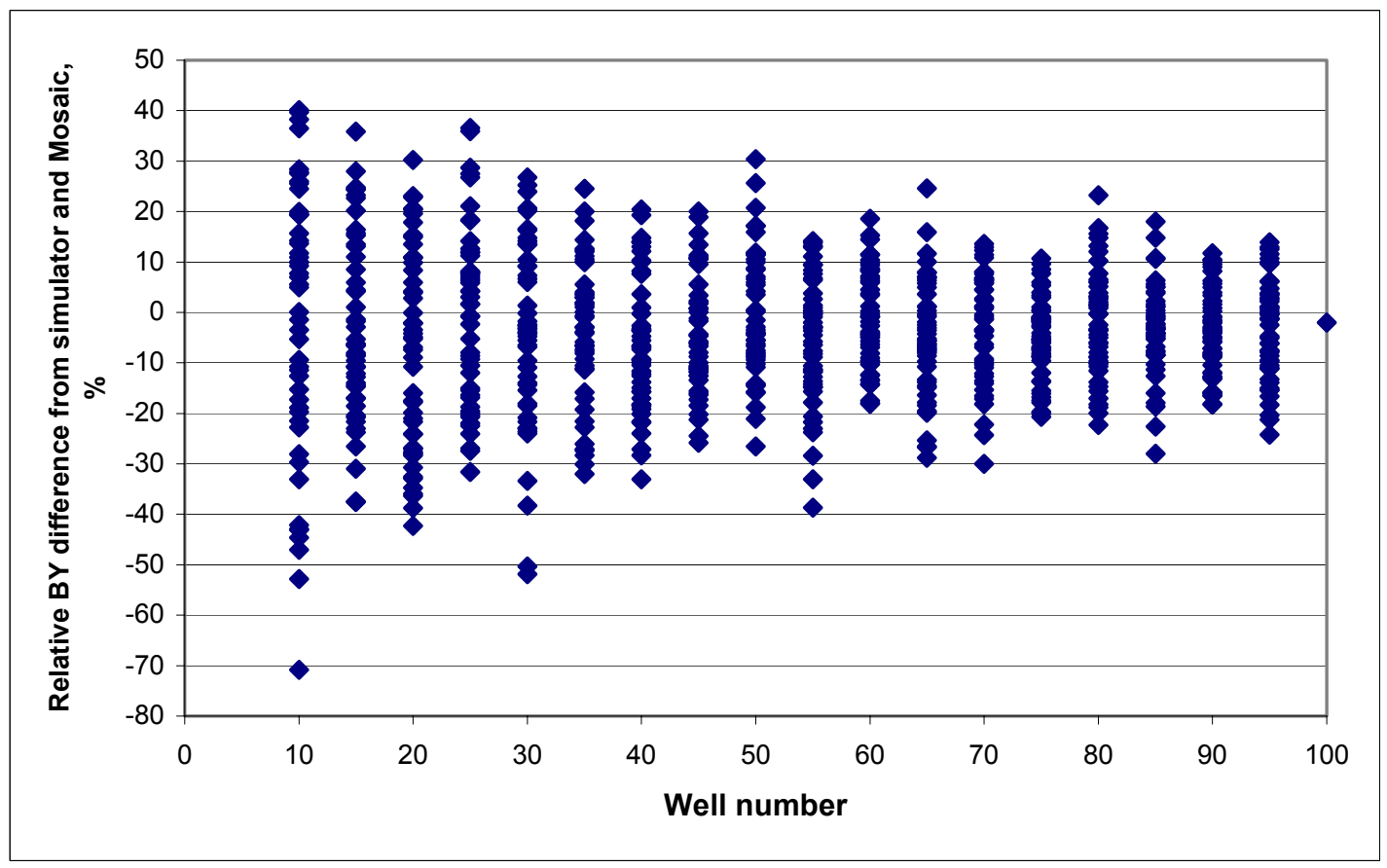

Fig. 4.16 Analysis of variability in error in average infill BY for Case 4. 
Similar analyses for all four cases are summarized in Fig. 4.17, which shows the standard deviation of each 50 subsets of $n$ wells for each of the four cases plotted on the same graph. As expected, the variability decreases as $n$ increases and the variability increases as the permeability heterogeneity increases.

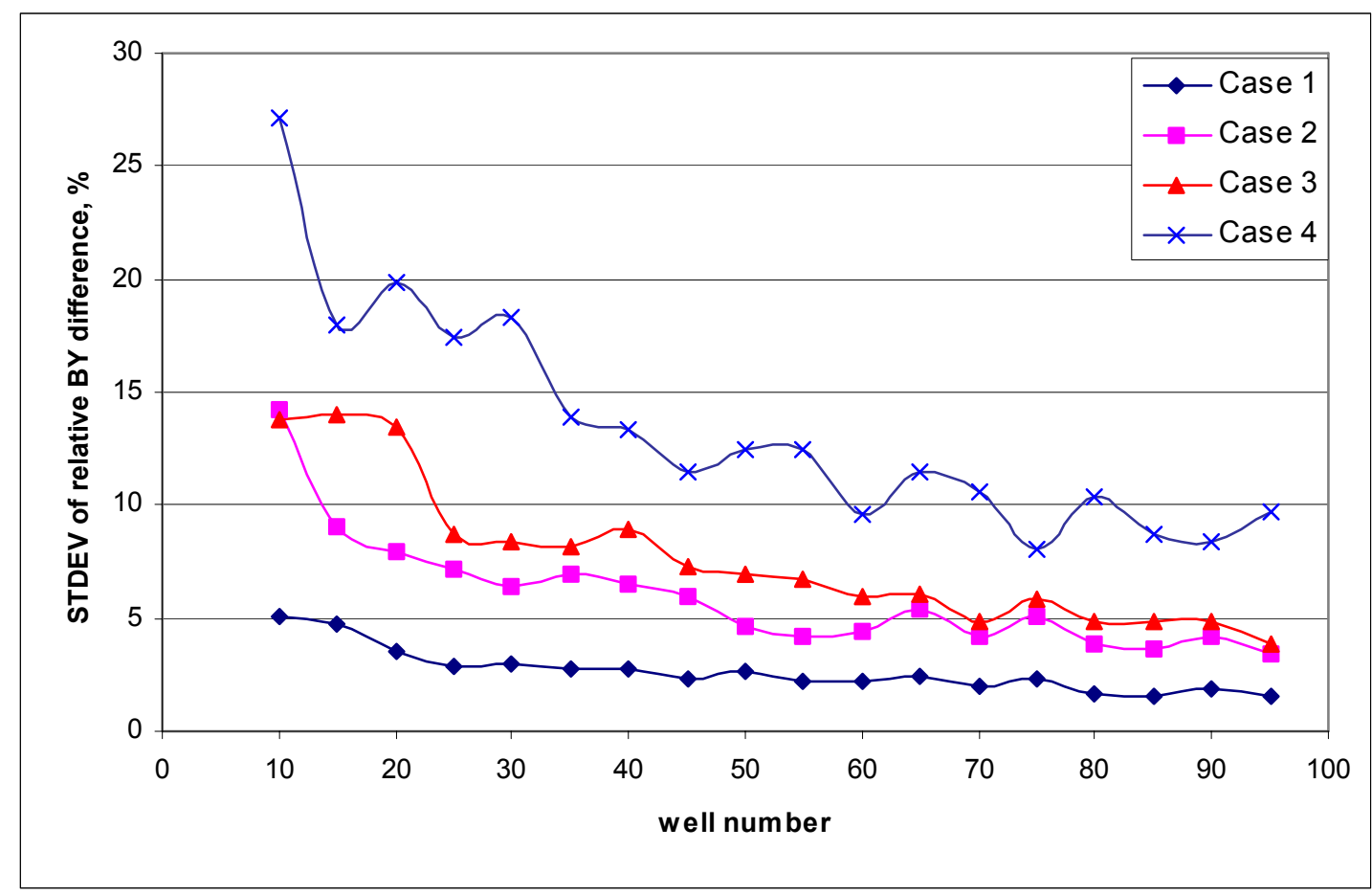

Fig. 4.17 Variability in percent error in average infill BY for four 100-well cases increases with reservoir heterogeneity and decreases with number of wells.

Fig. 4.18 is a similar plot, except that it shows the standard deviations of the absolute differences in averages of moving window and simulation infill BY as a function of n. The lines on Fig. 4.18 represent the theoretical behavior of the standard deviation, which assumes that the simulation and moving window averages are independent and that the standard deviation is proportional to $1 / \sqrt{n}$. Agreement between the model and calculations is good. Thus, we can estimate the number of wells 
required for a desired level of variability by applying the following procedure. This procedure is similar to that used for petrophysical sampling. ${ }^{11,12}$

- Pick 25 wells and evaluate the average and standard deviation of the best year, $\mathrm{BY}_{\mathrm{avg}}$ and $\mathrm{S}_{25}$, respectively.

- Choose the desired level of variability between BY avg and actual best year, $\tau$ (in $\%$ ), e.g., for $\pm 10 \%, \tau=10$. The desired level of variability means the mean of the 25 picked samples will be within $\pm 10 \%$ of the parent population mean for $95 \%$ of all possible samples.

- Evaluate the average for $n=\left[\left(206.4 \times S_{25}\right) /\left(\tau \times \mathrm{BY}_{\mathrm{avg}}\right)\right]^{2}$ wells. The estimation average for $n$ is based on the $\mathrm{I}_{0}$-Sampling approach ${ }^{12}$. Since we just pick 25 wells from the parent population, the $n$ is not a constant number because of sampling variability.

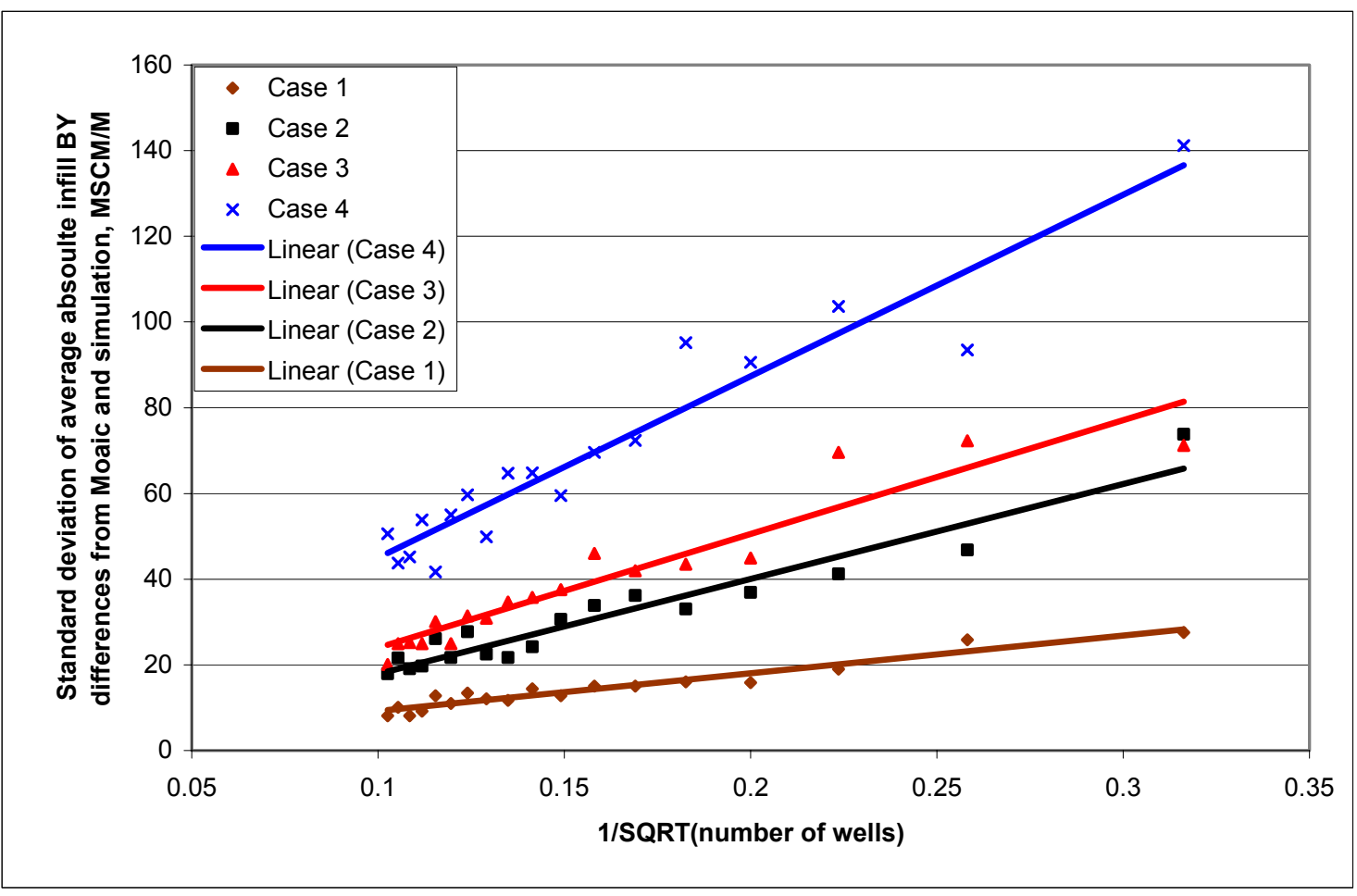

Fig. 4.18 Variability in absolute error in average infill BY and theoretical model fit. 
Table 4-6 shows the results of the estimated number of wells required for the desired level of $10 \%$ variability by applying the procedure above. It is clear that, as the reservoir becomes more heterogeneous, more wells will be needed to get the same desired level of variability. To get the same accuracy of estimation we need almost the same number of wells for the most heterogeneous reservoir cases, Case 4 and Case $4 \mathrm{a}^{*}$. But we need fewer wells for the uniform spacing Case 2a and Case $3 \mathrm{a}$ than for nonuniform spacing Case 2 and Case 3. We did not do further tests or analyze the theoretical behavior of the standard deviation.

Table 4.6-Number of Wells Required for Desired Level of Variability

\begin{tabular}{|c|c|c|c|c|}
\hline Case & $\begin{array}{c}\text { BY } \\
\text { avg, } \\
\text { MSCM/M }\end{array}$ & $\begin{array}{c}S_{25,} \\
\text { MSCM/M }\end{array}$ & $\begin{array}{c}\tau, \\
\%\end{array}$ & $\begin{array}{c}n, \\
\text { integer }\end{array}$ \\
\hline 2 & 511.5 & 131.5 & 10 & 28 \\
\hline 3 & 484.5 & 216.2 & 10 & 85 \\
\hline 4 & 586.6 & 371.5 & 10 & 171 \\
\hline $2 \mathrm{a}$ & 379.6 & 53.1 & 10 & 8 \\
\hline $3 \mathrm{a}$ & 358.6 & 107.5 & 10 & 38 \\
\hline $4 \mathrm{a}^{*}$ & 313.3 & 201.7 & 10 & 177 \\
\hline
\end{tabular}




\section{CHAPTER V}

\section{CONCLUSIONS}

The following conclusions are made on the basis of this study.

1. The moving window technique described in this thesis accurately predicts infill well performance for a group of infill candidates, often to within $10 \%$.

2. Predicted infill potential for individual wells can be off by more than $50 \%$.

3. For the cases examined in this work, the predicted average infill performance was either very close to or less than the simulated infill performance. This suggests that the moving window technology tends to underestimate the infill drilling potential, providing a conservative estimate.

4. The accuracy of predicted infill well performance, for either individual wells or the average of a group of wells, decreases as heterogeneity increases.

5. Accuracy of predicted average infill well performance increases as the number of wells in the group increases.

6. The estimation error of the moving window technique is related to the time difference between the date for the new infill wells and the date of the last round of infill wells.

7. The search area used in the moving window technique should not be too large relative to the reservoir permeability correlation length.

8. The primary advantages of the moving window technique are its speed and its reliance upon only well location and production data. It can be used to conduct infill-screening studies of projects consisting of thousands of wells and can be used to evaluate an entire basin in a matter of man-days. 


\section{CHAPTER VI}

\section{DISCUSSION OF LIMITATIONS AND FUTURE WORK}

From my research on moving window technology described in this thesis we found this technology has certain limitations.

- This technology is based on material balance and the pseudosteady-state flow equation for vertical wells. The pseudosteady-state flow equation for horizontal wells is different from vertical wells. So if horizontal wells are in the reservoir, results from the moving domain technology may be inaccurate.

- This technology is developed mainly for single-phase flow of gas in tight-gas reservoirs. We do not know the accuracy of the results from this technology under multiphase flow conditions, such as from gas reservoirs with significant water production or from oil reservoirs.

- The default search area for this technology is 3,000 acres. From the results of comparison of Case $4 \mathrm{a}$ and Case $4 \mathrm{a}^{*}$ (Table 4-3), we know the search area of 3,000 acres is too large for highly heterogeneous reservoirs. So we need a way to easily find the optimum search area.

Future work should focus on improving the accuracy of the moving window technology for single-phase gas flow. Efforts should focus on finding a way of determining the optimum parameters used in the moving domain technology. At the same time, we should try to determine whether this technology could be applied to a multiphase-flow reservoir. My suggestions on future work follow.

- We know the search area has significant effect on the accuracy of the estimation results. But we do not know how to determine the optimum search 
area. In our study cases we know the distribution of the reservoir permeability, but we do not know the distribution of reservoir permeability for actual reservoirs. Finding a way to determine the reservoir correlation length (and, thus, optimum search area) based on known reservoir parameters will be very helpful in improving the estimation accuracy of the moving window technology.

- The current well spacing is used as a proxy for the drainage area in the moving domain technology. The average current well spacing for the 100-well nonuniform well spacing cases is 535 acres. For the uniform well spacing cases, we first decreased the well spacing from 1,280 acres to 640 acres, then from 640 acres to 320 acres, and finally from 320 acres to 160 acres. Generally speaking, the estimation errors for the uniform spacing cases are less than for the 100-well non uniform spacing cases. It would be helpful to investigate further the effect of well spacing on estimation accuracy.

- From the limitations of the moving domain technology, we know this technology is developed mainly for low-permeability gas reservoirs. It would be helpful to conduct investigations in higher permeability reservoirs.

- The moving window technology is developed mainly for single-phase reservoirs. It would be helpful to expand the technology for use in reservoirs where multiphase flow is occurring. 


\section{NOMENCLATURE}

$$
\begin{array}{ll}
a_{0} & =\text { intermediate result in the reservoir model of moving window technology } \\
a_{1} & =\text { intermediate result in the reservoir model of moving window technology } \\
a_{2} & =\text { intermediate result in the reservoir model of moving window technology } \\
a_{3} & =\text { intermediate result in the reservoir model of moving window technology } \\
A & =\text { well spacing, acre } \\
\text { Avg } & =\text { abbreviation of average } \\
\text { APD } & =\text { average percentage difference, dimensionless }
\end{array}
$$

Avg_New_Well_BY= average new well 1-year cumulative production, the arithmetic average of all the cells in each well's simulation region, MSCM/M

$B \quad=$ formation volume factor, reservoir $\mathrm{ft}^{3} / \mathrm{scf}$

$\mathrm{BY}=$ best 12 consecutive months of production divided by $12, \mathrm{MSCM} / \mathrm{M}$

$\mathrm{BY}_{\mathrm{avg}}=$ average $\mathrm{BY}$ for 25 wells, MSCM/M

BY_Mosaic $=$ BY calculated from Mosaic technology, MSCM/M

$C_{1} \quad=$ intermediate result in the reservoir model of moving window technology

$C_{2} \quad=$ intermediate result in the reservoir model of moving window technology

$C_{3} \quad=$ intermediate result in the reservoir model of moving window technology

$C_{A} \quad=$ drainage area shape factor, dimensionless

$c_{t} \quad=$ total system compressibility, $\mathrm{psi}^{-1}$

$\mathrm{C}_{\mathrm{v}} \quad=$ coefficient of variation, dimensionless

D = time in day

DF $=$ depletion factor, $\%$

$d_{i} \quad=$ distance from the point $\left(x_{i}, y_{i}\right)$ to the unit slope line

$G_{p} \quad=$ cumulative production, standard cubic feet

$h \quad=$ thickness of the net pay, $\mathrm{ft}$ 


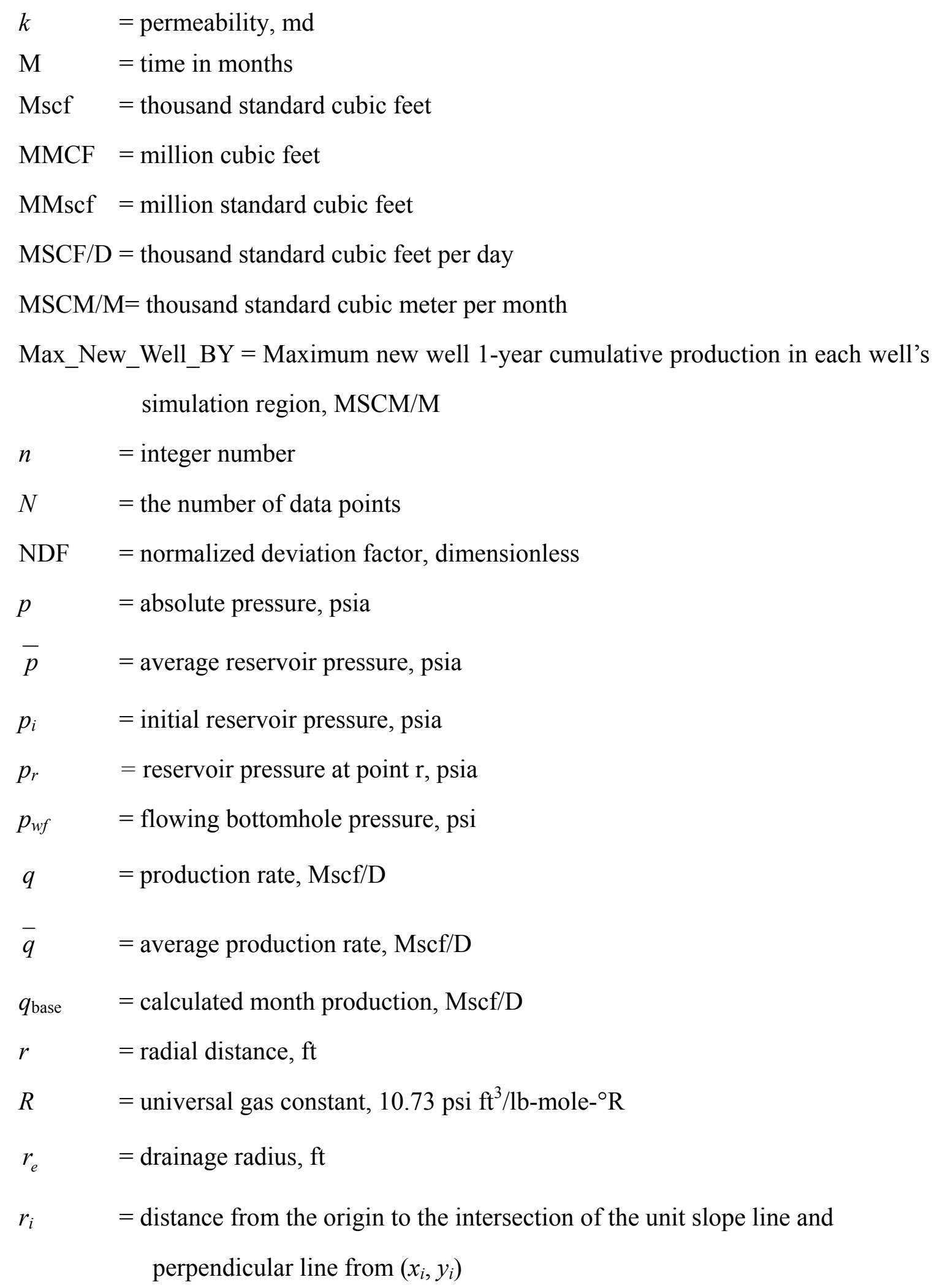




$$
\begin{array}{ll}
r_{w} & =\text { wellbore radius, } \mathrm{ft} \\
\mathrm{S} & =\text { well skin factor, dimensionless } \\
S_{25} & =\text { standard deviation of average BY for } 25 \text { wells, MSCM/M } \\
\tau & =\text { desired level of variability between infill BY from Mosaic and simulator, \% } \\
\text { STDEV } & =\text { standard deviation, dimensionless } \\
T & =\text { temperature, }{ }^{\circ} \mathrm{F} \\
x_{1} & =\text { variable in the reservoir model of moving window technology } \\
x_{2} & =\text { variable in the reservoir model of moving window technology } \\
x_{3} & =\text { variable in the reservoir model of moving window technology } \\
V & =\text { volume, } \mathrm{ft}^{3} \\
\mathrm{VBY} & =\text { BY corrected to a time before depletion effects, MSCM/M } \\
\text { VBY_Mosaic = the VBY calculated from Mosaic technology, MSCM/M } & =\text { gas compressibility, dimensionless } \\
Z & =\text { viscosity, cp } \\
\mu & =\text { porosity, fraction } \\
\pi & =
\end{array}
$$




\section{REFERENCES}

1. French, R.L., Brimhall, R.M., and Wu, C.H.: "A Statistical and Economic Analysis of Incremental Waterflood Infill Drilling Recoveries in West Texas Carbonate Reservoirs," paper SPE 22624 presented at the 1991 SPE Annual Technical Conference and Exhibition, Dallas, 6-9 October.

2. Wu, C.H., Lu, G.F., Gillespie, W., and Yen, J.: "Statistical and Fuzzy Infill Drilling Models for Carbonate Reservoirs," paper SPE 37728 presented at the 1997 SPE Middle East Oil Show \& Conference, Bahrain, 15-18 March.

3. Soto, B.R., Wu, C.H, and Buleba, A.M.: "Infill Drilling Recovery Models for Carbonate Reservoirs-A Multiple Statistical, Non-Parametric Regression, and Neural Network Approach,” paper SPE 57458 presented at the 1999 SPE Eastern Regional Conference and Exhibition, Charleston, West Virginia, 21-22 October.

4. McCain, W.D. Jr., Voneiff, G.W., Hunt, E.R., and Semmelbeck, M.E.: “A Tight Gas Field Study: Carthage (Cotton Valley) Field," paper SPE 26141 presented at the 1993 SPE Gas Technology Symposium, Calgary, 28-30 June.

5. Voneiff, G.W. and Cipolla, C.: "A New Approach to Large-Scale Infill Evaluations Applied to the Ozona (Canyon) Gas Field," paper SPE 35203 presented at the 1996 SPE Permian Oil and Gas Recovery Conference, Midland, Texas, 27-29 March.

6. Weber, K.J., and VanGenus, L.C.: "Framework for Constructing Clastic Reservoir Simulation Models," Journal of Petroleum Technology, 42 (1990).

7. Hecker, M.T. and Downie, R. C.: "Process Changes Improve Fracture Treatment Designs in the Hugoton Gas Field," paper SPE 35259 presented at the 1996 Gas Technology Conference, Calgary, 28 April - 1 May.

8. Hudson, J.W., Jochen, J.E., and Jochen, V.A.: "Practical Technique To Identify Infill Potential in Low-Permeability Gas Reservoirs Applied to the Milk River Formation 
in Canada," paper SPE 59779 presented at the 2000 SPE/CERI Gas Symposium, Calgary, 3-5 April.

9. Hudson, J.W., Jochen, J.E., and Spivey, J.P.: "Practical Methods to High-Grade Infill Opportunities Applied to the Mesaverde, Morrow, and Cotton Valley Formations," paper SPE 68598 presented at the 2001 SPE Hydrocarbon Economics and Evaluation Symposium, Dallas, 2-3 April 2001.

10. Kyte, D.G. and Meehan, D.N.: "Horizontal Spacing, Depletion, and Infill Potential in the Austin Chalk," paper SPE 36721 presented at the 1996 SPE Annual Technical Conference and Exhibition, Denver, 6-9 October.

11. Dake, L.P., Fundamentals of Reservoir Engineering, Elsevier, Amsterdam, (1978).

12. Jensen, J.L., Lake, L.W, Corbett, P.W.M., and Goggin, D.J.: Statistics for Petroleum Engineers and Geoscientists, second edition, Elsevier, Amsterdam (2000). 


\section{APPENDIX A}

\section{MOSAIC 4D MODEL FOR GAS RESERVOIR}

After a well produces at constant rate for a period of time, the reservoir boundary effects interrupt the infinite-acting pressure behavior. If the well is in an irregularly shaped drainage area, the closest boundary to the well causes the earliest departure from the infinite-acting reservoir.

When the reservoir boundary begins to have a significant effect on well drawdown, the transient region ends and the pseudosteady-state region begins. When the reservoir pressure starts to decline at the same rate at all points in the reservoir, this condition is often referred to as "pseudosteady-state."

From the radial diffusivity equation, the pressure $p$ at any point $r$ in a reservoir of radius $r_{\mathrm{e}}$ is given by Eq.A- $1^{11}$.

$$
p=p_{w f}+\frac{141.2 \times q \times B \times \mu}{k \times h}\left(\ln \left(\frac{r}{r_{w}}\right)-\frac{r^{2}}{2 \times r_{e}^{2}}\right)
$$

at $r=r_{e}$, the above equation can be converted to

$$
p_{e}=p_{w f}+\frac{141.2 \times q \times B \times \mu}{k \times h}\left(\ln \left(\frac{r_{e}}{r_{w}}\right)-\frac{1}{2}\right)
$$

By definition the average reservoir pressure can be calculated by Eq.A-3.

$$
\bar{p}=\frac{\int_{r_{w}}^{r_{e}} p d v}{\pi \times\left(r_{e}{ }^{2}-r_{w}{ }^{2}\right) \times h \times \phi}
$$

Because $r_{e}>>r_{w}$, Eq.A-3 becomes

$$
\bar{p}=\frac{\int_{r_{w}}^{r_{e}} p d v}{\pi \times\left(r_{e}{ }^{2}-r_{w}{ }^{2}\right) \times h \times \phi} \approx \frac{\int_{r_{w}}^{r_{e}} p d v}{\pi \times\left(r_{e}{ }^{2}\right) \times h \times \phi}
$$


Where we can get $V$ and $d v$ from the following Eqs. A-5 and A-6.

$V=\pi \times r^{2} \times h \times \phi$

$d v=2 \pi \times r \times h \times \phi \times d r$

So, Eq. A-4 becomes

$\bar{p}=\frac{2}{r_{e}{ }^{2}} \int_{r_{w}}^{r_{e}} p_{r} d r$

Combining Eq. A-7 and Eq. A-1 we have

$\bar{p}-p_{w f}=\frac{2}{r_{e}{ }^{2}} \int_{r_{w}}^{r_{e}} \frac{141.2 \times q \times B \times \mu}{k \times h}\left(\ln \left(\frac{r}{r_{w}}\right)-\frac{r}{2 r_{e}{ }^{2}}\right) d r$

If we simplify Eq. A-8 we have

$$
\bar{p}-p_{w f}=\frac{141.2 \times q \times B \times \mu}{k \times h}\left(\ln \left(\frac{r_{e}}{r_{w}}\right)-\frac{3}{4}\right)
$$

If we take the skin factor into consideration, Eq. A-9 becomes

$$
\bar{p}-p_{w f}=\frac{141.2 \times q \times B \times \mu}{k \times h}\left(\ln \left(\frac{r_{e}}{r_{w}}\right)-\frac{3}{4}+s\right)
$$

When we want to use drainage area, $A$, and generalized reservoir geometry factor, $C_{A}$, in the above equation we need to do the following transforms. We assume the drainage area is a circle. Then $C_{A}$ is equal to 31.6 .

$$
\begin{aligned}
& \ln \left(\frac{r_{e}}{r_{w}}\right)=\frac{1}{2} \ln \left(\frac{r_{e}}{r_{w}}\right)^{2}=\frac{1}{2} \ln \left(\frac{\pi \times r_{e}{ }^{2}}{\pi \times r_{w}{ }^{2}}\right)=\frac{1}{2} \ln \left(\frac{A}{\pi \times r_{w}{ }^{2}}\right) \\
& \ln \left(\frac{r_{e}}{r_{w}}\right)=\frac{1}{2} \ln \left(\frac{A}{r_{w}{ }^{2}} \times \frac{1}{\pi}\right)=\frac{1}{2} \ln \left(\frac{A}{r_{w}{ }^{2}} \times \frac{10.06}{C_{A}}\right)
\end{aligned}
$$

Here we get the following Eq. A-13.

$$
\bar{p}-p_{w f}=\frac{141.2 \times q \times B \times \mu}{k \times h}\left(\frac{1}{2} \ln \left(\frac{10.06 A}{C_{A} \times r_{w}{ }^{2}}\right)-\frac{3}{4}+s\right)
$$


When we rewrite the above equation we have

$$
q=\frac{\left(\bar{p}-p_{w f}\right) \times k \times h}{141.2 \times B \times \mu\left(\frac{1}{2} \ln \left(\frac{10.06 A}{C_{A} \times r_{w}{ }^{2}}\right)-\frac{3}{4}+s\right)}
$$

Now we will use $p_{\text {i }}$ to express $\bar{p}$.

By definitions of compressibility and volume we have Eq. A-15 and A-16.

$$
\begin{aligned}
& c_{t}=-\frac{1}{V} \frac{d V}{d p} \quad \ldots \\
& V=\pi \times r_{e}^{2} \times h \times \phi
\end{aligned}
$$

From Eq.A-15 we can get

$$
c_{t} \times V \times d p=-d V
$$

Combing Eq. A-16 and Eq. A-17 we have

$$
\pi \times c_{t} \times r_{e}^{2} \times h \times \phi \times \int_{p_{i}}^{\bar{p}} d p=-\int_{0}^{V} d V
$$

$V$ is the volume of the cumulative production oil. If we express it in terms of average production and time we have

$$
\pi \times c_{t} \times r_{e}^{2} \times h \times \phi \times\left(\bar{p}-p_{i}\right)=-V=-\bar{q} \times B \times t
$$

Simplifying the above equation we have

$$
\left(\bar{p}-p_{i}\right)=-\frac{\bar{q} \times B \times t}{\pi \times c_{t} \times r_{e}^{2} \times h \times \phi}
$$

If we change the units of time and volume from hours to days and barrels to cubic feet, Eq. A-20 becomes

$$
\left(\bar{p}-p_{i}\right)=-\frac{\bar{q} \times B \times t}{\pi \times c_{t} \times r_{e}{ }^{2} \times h \times \phi} \times \frac{1}{24 \times 5.615}=-0.00742 \frac{G_{p} \times B}{c_{t} \times r_{e}{ }^{2} \times h \times \phi}
$$


Here we have

$$
\bar{p}=p_{i}-0.00742 \frac{G_{p} \times B}{c_{t} \times r_{e}{ }^{2} \times h \times \phi}
$$

Combining Eq. A-14 and Eq. A-22 we have

$$
q=\frac{\left(p_{i}-0.00742 \frac{G_{p} \times B}{c_{t} \times r_{e}^{2} \times h \times \phi}-p_{w f}\right) k \times h}{141.2 \times B \times \mu\left(\frac{1}{2} \ln \left(\frac{10.06 A}{C_{A} \times r_{w}{ }^{2}}\right)-\frac{3}{4}+s\right)}
$$

Rearranging the above Eq. A-23 we have

$$
q=\frac{k h}{141.2 \times B \times \mu} \times \frac{\left(p_{i}-0.00742 \frac{G_{p} \times B}{c_{t} \times r_{e}{ }^{2} \times h \times \phi}-p_{w f}\right)}{\left(\frac{1}{2} \ln \left(\frac{10.06 A}{C_{A} \times r_{w}{ }^{2}}\right)-\frac{3}{4}+s\right)}
$$

In the moving window technique, we assume the following variables, $p_{i}, p_{w f}, B, \phi$, $c_{t}, h, \mu, C_{A}, r_{w}$, and $s$, are constants within each moving window. This assumes that, within each window, the reservoir is homogeneous and the properties of the reservoir fluids do not change significantly with time.

In the moving window technique, $q$ is taken as the best year, BY, and it can be calculated from the following equations. 


$$
\begin{aligned}
& \ln q=\ln \left(\frac{k h}{141.2 B \mu}\right)+\ln \left(p_{i}-0.00742 \frac{\pi G_{p} B}{c_{t} A h \phi}-p_{w f}\right)-\ln \left[\frac{1}{2} \ln \left(\frac{10.06 A}{C_{A} r_{w}{ }^{2}}\right)-\frac{3}{4}+s\right] \\
& =\ln (k h)-\ln (141.2 B \mu)+\ln \left(p_{i}-0.00742 \frac{\pi G_{p} B}{c_{t} A h \phi}-P_{w f}\right) \\
& -\ln \left\{\ln \left[\left(\frac{10.06 A}{C_{A} r_{w}{ }^{2}}\right)^{0.5}\right]-\frac{3}{4}+s\right\}
\end{aligned}
$$

where $\ln \left\{\ln \left[\left(\frac{10.06 A}{C_{A} r_{w}{ }^{2}}\right)^{0.5}\right]-\frac{3}{4}+s\right\}=$

$$
\begin{aligned}
& =\ln \left(0.5 \times \ln (10.06 \times A)-0.5 \times \ln \left(C_{A} \times r_{w}{ }^{2}\right)-\frac{3}{4}+s\right) \\
& =\ln \left(0.5 \times \ln (A)+0.5 \times \ln (10.06)-0.5 \times \ln \left(C_{A} \times r_{w}{ }^{2}\right)-\frac{3}{4}+s\right) \\
& =\ln \left(\ln (\sqrt{A})+C_{3}\right)
\end{aligned}
$$

Combining Eq.A-25 and A-26 we have

$\ln q=\ln (k h)+C_{1}+\ln \left(p_{i}-p_{w f}-C_{2} \frac{G_{p}}{A}\right)-\ln \left(\ln (\sqrt{A})+C_{3}\right)$

where

$$
\begin{aligned}
& C_{1}=\quad-\ln (141.2 B \mu) \\
& C_{2}=\quad 0.00742 \frac{\pi B}{c_{t} h \phi} \\
& C_{3}=\quad 0.40428+s+0.5 \ln \left(C_{A} r_{w}{ }^{2}\right)
\end{aligned}
$$


We can write Eq. A-27 in generalized form.

$y=a_{0}+a_{1} x_{1}+a_{2} x_{2}+a_{3} x_{3}$

Where

$$
\begin{aligned}
& a_{0}, a_{1}, a_{2}, \text { and } a_{3} \text { are constant for a moving window. } \\
& y=\ln (q) \\
& x_{1}=\ln (k h) \\
& x_{2}=\ln \left(G_{p} / A\right) \\
& x_{3}=\ln (\ln (\sqrt{A}))
\end{aligned}
$$

Eq. A-28 is the Mosaic 4D equation that we use in the moving window technology. In applying this equation, we use BY in place of $q$, VBY as a proxy for $k h$, and the well spacing of the well as its drainage area. 


\section{VITA}

Name: $\quad$ Linhua Guan

Born: $\quad 19$ June 1971

Jinzhou, P.R. China

Permanent Address: Datun, Jiudaoling, Yi Xian

Jinzhou, Liaoning Province, P.R.C. 121102

Education: $\quad$ Texas A\&M University, College Station, Texas, USA

Master of Science in Petroleum Engineering

May 2003

University of Petroleum of China, P.R. China

Master of Science in Petroleum Geology, June 1996

University of Petroleum of China, P.R. China

Bachelor of Science in Well Logging, July 1993

Experience: Geological Engineer \& Petrophysicist, Comprehensive Research Department, China Petroleum Economics and Information Research Center, P.R. China, July 1996 - August 2000

Affiliations: $\quad$ Society of Petroleum Engineers 